Supporting Information

for

\title{
Dinitrogen Cleavage by a Heterometallic Cluster Featuring Multiple Uranium-Rhodium Bonds
}

Xiaoqing Xin, ${ }^{[a]}$ Iskander Douair, ${ }^{[b]}$ Yue Zhao, ${ }^{[a]}$ Shuao Wang, ${ }^{[c]}$ Laurent Maron, ${ }^{* b]}$ and Congqing Zhu*[a]

${ }^{[a]}$ State Key Laboratory of Coordination Chemistry, Jiangsu Key Laboratory of Advanced Organic Materials, School of Chemistry and Chemical Engineering, Nanjing University, Nanjing 210023, China ${ }^{[b]}$ LPCNO, CNRS \& INSA, Université Paul Sabatier, 135 Avenue de Rangueil, 31077 Toulouse, France ${ }^{[c]}$ State Key Laboratory of Radiation Medicine and Protection, School for Radiological and interdisciplinary Sciences (RAD-X) and Collaborative Innovation Center of Radiation Medicine of Jiangsu Higher Education Institutions, Soochow University, Suzhou, China

*Correspondence and requests for materials should be addressed to L.M. (E-mail: laurent.maron@irsamc.ups-tlse.fr) or to C.Z. (E-mail: zcq@nju.edu.cn).

\section{Contents}

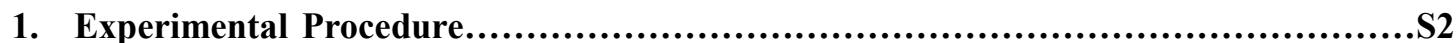

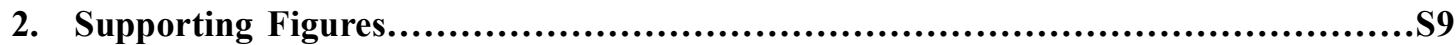

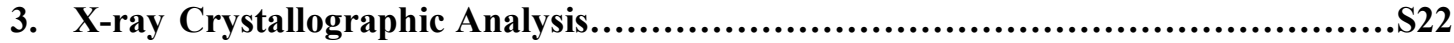

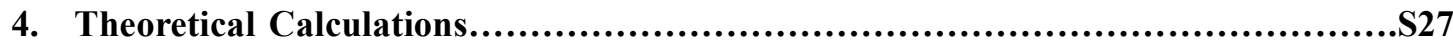

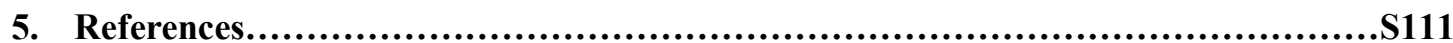




\section{Experimental Procedure}

General Procedure: All manipulations were performed under an $\mathrm{N}_{2}$ atmosphere using standard Schlenk techniques or in a glovebox. Commercially available chemicals were used as received without further purification. The solvents were obtained by passing through a Solve Purer G5 (MIKROUNA) solvent purification system and further dried over $4 \AA$ molecular sieves. Benzene$\mathrm{d}_{6}$ and THF- $\mathrm{d}_{8}$ were dried over $\mathrm{Na} / \mathrm{K}$ and stored under a $\mathrm{N}_{2}$ atmosphere prior to use. Nuclear magnetic resonance spectroscopy was performed using a Bruker AVIII-400 $\left({ }^{1} \mathrm{H} 400 \mathrm{MHz} ;{ }^{13} \mathrm{C}\left\{{ }^{1} \mathrm{H}\right\}\right.$ $101 \mathrm{MHz} ;{ }^{31} \mathrm{P}\left\{{ }^{1} \mathrm{H}\right\} 162 \mathrm{MHz}$ ) spectrometer at room temperature (RT). The ${ }^{1} \mathrm{H}$ and ${ }^{13} \mathrm{C}\left\{{ }^{1} \mathrm{H}\right\} \mathrm{NMR}$ chemical shifts $(\delta)$ are relative to tetramethylsilane, and ${ }^{31} \mathrm{P}\left\{{ }^{1} \mathrm{H}\right\}$ NMR chemical shifts are relative to $85 \% \mathrm{H}_{3} \mathrm{PO}_{4}$. Absolute values of the coupling constants are provided in $\mathrm{Hertz}(\mathrm{Hz})$. Multiplicities are abbreviated as singlet (s), doublet (d), triplet (t), multiplet (m), and broad (br). Magnetic susceptibility measurements on crystalline samples were performed using a Quantum Design SQUID VSM magnetometer from 300 to $2 \mathrm{~K}$ under an external magnetic field of 1000 Oe. Recrystallized powdered samples were used for the measurements to ensure the purity of samples. Data reproducibility was examined by reproduced experiments for each compound. All the data were corrected for the underlying diamagnetic increment $\left(364.25 \times 10^{-6} \mathrm{~cm}^{3} \mathrm{~mol}^{-1}(2), 725.5 \times 10^{-6}\right.$ $\mathrm{cm}^{3} \mathrm{~mol}^{-1}(3), 690 \times 10^{-6} \mathrm{~cm}^{3} \mathrm{~mol}^{-1}(\mathbf{4})$ and $\left.1287.5 \times 10^{-6} \mathrm{~cm}^{3} \mathrm{~mol}^{-1}(\mathbf{5})\right)$ and the effect of the sample holder (gelatine capsule) and core diamagnetism of the sample using Pascal's constants. Absorption spectra were measured on a Lambda 750 spectrometer at room temperature. Elemental analyses $(\mathrm{C}$, $\mathrm{H}, \mathrm{N}$ ) were performed on a Vario EL III elemental analyzer at the Shanghai Institute of Organic Chemistry, Chinese Academy of Sciences. High-resolution mass spectroscopy (HRMS) was conducted using a Waters SYNAPT G2-Si system equipped with an ESI ionization source. 


\section{Synthesis of $\left[\mathrm{CH}_{3} \mathrm{~N}\left(\mathrm{CH}_{2} \mathrm{CH}_{2} \mathrm{NHP}^{i} \mathrm{Pr}_{2}\right)_{2}\right](1)$}

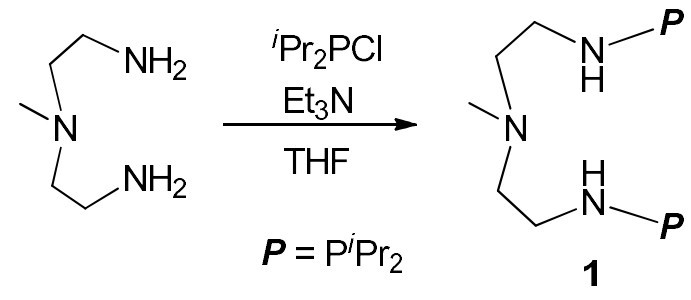

A solution of ${ }^{i} \mathrm{Pr}_{2} \mathrm{PCl}$ (3.06 g, $20.0 \mathrm{mmol}, 2$ equiv.) in THF ( $\left.20 \mathrm{~mL}\right)$ was added dropwise to a solution of $\mathrm{CH}_{3} \mathrm{~N}\left(\mathrm{CH}_{2} \mathrm{CH}_{2} \mathrm{NH}_{2}\right)_{2}(1.17 \mathrm{~g}, 10.0 \mathrm{mmol}, 1$ equiv. $)$ and $\mathrm{Et}_{3} \mathrm{~N}(11 \mathrm{~mL}, 80.0 \mathrm{mmol}, 8$ equiv. $)$ in THF $(30 \mathrm{~mL})$, resulting in the immediate formation of a white precipitate. The mixture was stirred overnight then dried in vacuo. The white solid was extracted with hexane and filtered through celite. The volatile components were removed under reduced pressure to give compound $\mathbf{1}$ as a colorless oil. Yield: $3.14 \mathrm{~g}(90 \%) .{ }^{1} \mathrm{H}$ NMR $\left(\mathrm{C}_{6} \mathrm{D}_{6}, 400 \mathrm{MHz}, \mathrm{ppm}\right) \delta$ 2.92-2.98 (m, 4H, $\left.\mathrm{NCH}_{2} \mathrm{CH}_{2}\right), 2.25$ (t, $\left.{ }^{3} J_{\mathrm{HH}}=6.4 \mathrm{~Hz}, 4 \mathrm{H}, \mathrm{NCH}_{2} \mathrm{CH}_{2}\right), 2.02\left(\mathrm{~s}, 3 \mathrm{H}, \mathrm{NCH}_{3}\right), 1.44-1.55\left(\mathrm{~m}, 4 \mathrm{H}, \mathrm{CH}\left(\mathrm{CH}_{3}\right)_{2}\right), 1.31-1.33(\mathrm{~m}$, $2 \mathrm{H}, \mathrm{NH}), 1.00-1.06\left(\mathrm{~m}, 24 \mathrm{H}, \mathrm{CH}\left(\mathrm{CH}_{3}\right)_{2}\right) .{ }^{13} \mathrm{C}\left\{{ }^{1} \mathrm{H}\right\} \mathrm{NMR}\left(\mathrm{C}_{6} \mathrm{D}_{6}, 101 \mathrm{MHz}, \mathrm{ppm}\right) \delta 60.5\left(\mathrm{NCH}_{2} \mathrm{CH}_{2}\right)$, $60.4\left(\mathrm{NCH}_{2} \mathrm{CH}_{2}\right), 46.2\left(\mathrm{NCH}_{2} \mathrm{CH}_{2}\right), 45.9\left(\mathrm{NCH}_{2} \mathrm{CH}_{2}\right), 41.6\left(\mathrm{CH}_{3} \mathrm{~N}\right), 26.5\left(\mathrm{CH}\left(\mathrm{CH}_{3}\right)_{2}\right), 26.4$ $\left(\mathrm{CH}\left(\mathrm{CH}_{3}\right)_{2}\right) 19.2\left(\mathrm{CH}\left(\mathrm{CH}_{3}\right)_{2}\right), 19.0\left(\mathrm{CH}\left(\mathrm{CH}_{3}\right)_{2}\right), 17.4\left(\mathrm{CH}\left(\mathrm{CH}_{3}\right)_{2}\right), 17.3\left(\mathrm{CH}\left(\mathrm{CH}_{3}\right)_{2}\right) .{ }^{31} \mathrm{P}\left\{{ }^{1} \mathrm{H}\right\} \mathrm{NMR}$ $\left(\mathrm{C}_{6} \mathrm{D}_{6}, 162 \mathrm{MHz}, \mathrm{ppm}\right) \delta$ 63.6. HRMS (ESI) calcd for $\mathrm{C}_{17} \mathrm{H}_{41} \mathrm{~N}_{3} \mathrm{P}_{2}[\mathrm{M}+\mathrm{H}]^{+}$350.2848, found 350.2872 .

Synthesis of $\left\{\mathrm{U}\left[\mathrm{N}\left(\mathrm{CH}_{3}\right)\left(\mathrm{CH}_{2} \mathrm{CH}_{2} \mathrm{NP}^{i} \mathrm{Pr}_{2}\right)_{2}\right](\mathrm{Cl})_{2}(\mathrm{THF})\right\}$ (2)<smiles></smiles>

1 $\stackrel{\text { i) }{ }^{n} \mathrm{BuLi}, \mathrm{THF}}{\longrightarrow}$

ii) $\mathrm{UCl}_{4}, \mathrm{THF}$ $P=P^{i} P_{2}$

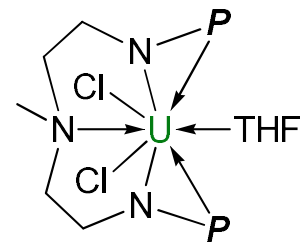

2 
A $2.4 \mathrm{M}$ solution of $n$-BuLi in hexane $(1.68 \mathrm{~mL}, 4.0 \mathrm{mmol}, 2$ equiv.) was added dropwise to a solution of compound $\mathbf{1}\left(700 \mathrm{mg}, 2.0 \mathrm{mmol}, 1\right.$ equiv.) in THF $(20 \mathrm{~mL})$ at $-30{ }^{\circ} \mathrm{C}$. The mixture was allowed to warm to RT and stirred for a further $2 \mathrm{~h}$, and was then added to the solution of $\mathrm{UCl}_{4}(760$ $\mathrm{mg}, 2.0 \mathrm{mmol}, 1$ equiv.) in THF $(20 \mathrm{~mL})$. The mixture was stirred overnight at RT and then the solvents were removed under reduced pressure and the residues were extracted with toluene. The filtrate was dried in vacuo to afford complex 2 as a pure brown solid $(1.19 \mathrm{~g}, 82 \%)$. Crystals of 2 suitable for X-ray diffraction were grown from a saturated solution in toluene stored at $-30{ }^{\circ} \mathrm{C} .{ }^{1} \mathrm{H}$

$\operatorname{NMR}\left(\mathrm{C}_{6} \mathrm{D}_{6}, 400 \mathrm{MHz}, \mathrm{ppm}\right) \delta 88.49\left(\mathrm{~s}, 2 \mathrm{H}, \mathrm{CH}_{2}\right), 72.02\left(\mathrm{~s}, 2 \mathrm{H}, \mathrm{CH}_{2}\right), 69.15\left(\mathrm{~s}, 2 \mathrm{H}, \mathrm{CH}_{2}\right), 58.14$ (s, 2H, $\left.\mathrm{CH}_{2}\right), 32.55$ (s, 6H, $\left.\mathrm{CH}\left(\mathrm{CH}_{3}\right)_{2}\right), 32.03$ (s, 6H, $\left.\mathrm{CH}\left(\mathrm{CH}_{3}\right)_{2}\right), 29.00$ (s, 6H, $\left.\mathrm{CH}\left(\mathrm{CH}_{3}\right)_{2}\right), 25.90$ (s, 6H, $\left.\mathrm{CH}\left(\mathrm{CH}_{3}\right)_{2}\right),-21.25(\mathrm{~s}, 4 \mathrm{H}, \mathrm{THF}),-36.13\left(\mathrm{~s}, 2 \mathrm{H}, \mathrm{CH}\left(\mathrm{CH}_{3}\right)_{2}\right),-52.90$ (s, 4H, THF), -70.89 (s, $\left.2 \mathrm{H}, \mathrm{CH}\left(\mathrm{CH}_{3}\right)_{2}\right),-81.53\left(\mathrm{~s}, 3 \mathrm{H}, \mathrm{NCH} H_{3}\right) .{ }^{13} \mathrm{C}\left\{{ }^{1} \mathrm{H}\right\} \mathrm{NMR}\left(\mathrm{C}_{6} \mathrm{D}_{6}, 101 \mathrm{MHz}, \mathrm{ppm}\right) \delta 30.7,29.0,21.8$, 13.1, 0.2. ${ }^{31} \mathrm{P}\left\{{ }^{1} \mathrm{H}\right\}$ NMR $\left(\mathrm{C}_{6} \mathrm{D}_{6}, 162 \mathrm{MHz}, \mathrm{ppm}\right)$ not observed from the range of +1000 to -1000 ppm. Anal. Calcd. for $\mathrm{C}_{21} \mathrm{H}_{47} \mathrm{Cl}_{2} \mathrm{~N}_{3} \mathrm{OP}_{2} \mathrm{U}$ : C, 34.62; H, 6.50; N, 5.77. Found: C, 34.42; H, 6.47; N, 5.83 .

\section{Synthesis of $\left[\left\{\mathrm{U}\left[\mathrm{N}\left(\mathrm{CH}_{3}\right)\left(\mathrm{CH}_{2} \mathrm{CH}_{2} \mathrm{NP}^{i} \mathrm{Pr}_{2}\right)_{2}\right]\left(\mathrm{Cl}_{2}\right)\right\}_{2}(\mu-\mathrm{Cl})(\mu-\mathrm{Rh})\right](3)$}

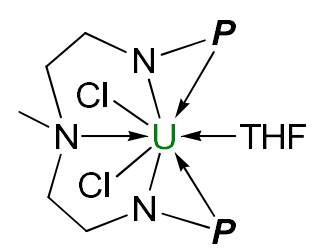

2

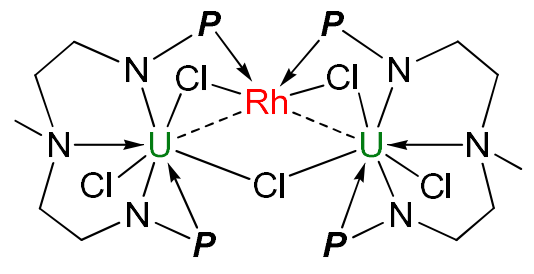

3

A toluene solution $(2 \mathrm{~mL})$ of $[\mathrm{RhCl}(\mathrm{COD})]_{2}(24.7 \mathrm{mg}, 0.05 \mathrm{mmol}, 1$ equiv.) was added to a stirred solution of 2 (145.7 mg, $0.2 \mathrm{mmol}, 4$ equiv.) in toluene $(2 \mathrm{~mL})$ at RT. The reaction was heated at $110^{\circ} \mathrm{C}$ for $4 \mathrm{~h}$, then the mixture was filtered through a sintered glass funnel and the filtrate was 
concentrated to $2 \mathrm{~mL}$. After the concentrated solution was held at $-30^{\circ} \mathrm{C}$ for $24 \mathrm{~h}$, brown crystals of 3 suitable for X-ray diffraction were obtained (50.8 mg, 35\%). ${ }^{1} \mathrm{H}$ NMR (THF- $\left.\mathrm{d}_{8}, 400 \mathrm{MHz}, \mathrm{ppm}\right)$ $\delta 96.22(\mathrm{~s}, 2 \mathrm{H}), 82.57(\mathrm{~s}, 2 \mathrm{H}), 68.09(\mathrm{~s}, 2 \mathrm{H}), 59.77(\mathrm{~s}, 2 \mathrm{H}), 53.62(\mathrm{~s}, 2 \mathrm{H}), 49.33(\mathrm{~s}, 2 \mathrm{H}), 36.55(\mathrm{~s}$, 2H), $32.47(\mathrm{~s}, 6 \mathrm{H}), 28.85\left(\mathrm{~d},{ }^{3} J_{\mathrm{HH}}=7.6 \mathrm{~Hz}, 6 \mathrm{H}\right), 28.18(\mathrm{~s}, 6 \mathrm{H}), 24.56(\mathrm{~s}, 2 \mathrm{H}), 24.19\left(\mathrm{~d},{ }^{3} J_{\mathrm{HH}}=5.2\right.$ $\mathrm{Hz}, 6 \mathrm{H}), 23.58\left(\mathrm{~d},{ }^{3} J_{\mathrm{HH}}=7.2 \mathrm{~Hz}, 6 \mathrm{H}\right), 18.83\left(\mathrm{~d},{ }^{3} J_{\mathrm{HH}}=6.8 \mathrm{~Hz}, 6 \mathrm{H}\right), 18.04\left(\mathrm{~d},{ }^{3} J_{\mathrm{HH}}=7.6 \mathrm{~Hz}, 6 \mathrm{H}\right)$, $3.91(\mathrm{~s}, 6 \mathrm{H}),-31.96(\mathrm{~s}, 2 \mathrm{H}),-42.55(\mathrm{~s}, 2 \mathrm{H}),-66.58(\mathrm{~s}, 2 \mathrm{H}),-75.32(\mathrm{~s}, 6 \mathrm{H}),-82.57(\mathrm{~s}, 2 \mathrm{H}) .{ }^{31} \mathrm{P}\left\{{ }^{1} \mathrm{H}\right\}$ NMR (THF-d $8,162 \mathrm{MHz}, \mathrm{ppm}) \delta 556.6(\mathrm{br}), 64.2(\mathrm{~s}) .{ }^{13} \mathrm{C}$ NMR spectrum could not be obtained due to its low solubility. Anal. Calcd. for $\mathrm{C}_{34} \mathrm{H}_{78} \mathrm{Cl}_{5} \mathrm{~N}_{6} \mathrm{P}_{4} \mathrm{RhU}_{2}$ 2toluene: C 35.25; H 5.79; N 5.14. Found: C 34.30; H 5.82; N 5.32.

\section{Synthesis of $\left[\left\{\mathrm{U}\left[\mathrm{N}\left(\mathrm{CH}_{3}\right)\left(\mathrm{CH}_{2} \mathrm{CH}_{2} \mathrm{NP}^{i} \mathrm{Pr}_{2}\right)_{2}\right](\mathrm{Cl})\right\}_{2}(\mu-\mathrm{Cl})(\mu-\mathrm{Rh})\right](4)$}

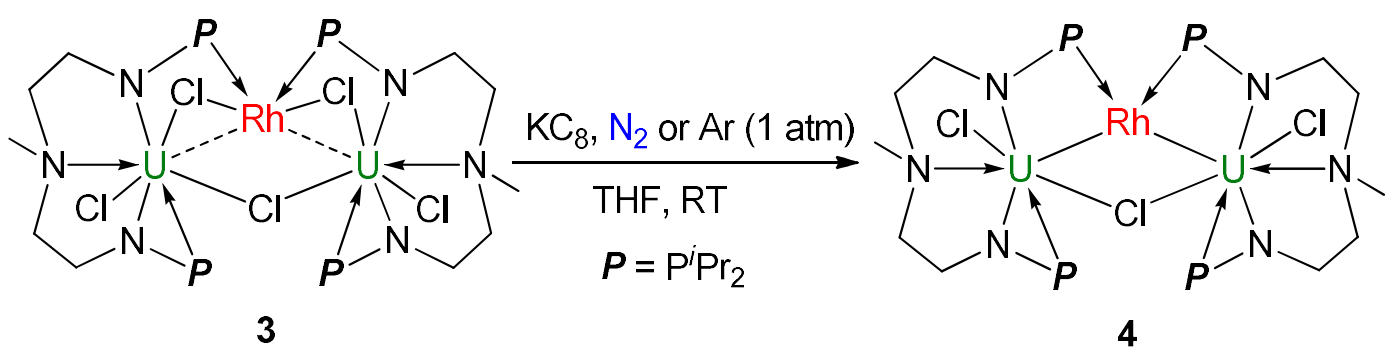

A slurry of $\mathrm{KC}_{8}(8.1 \mathrm{mg}, 0.06 \mathrm{mmol}, 2$ equiv.) in THF was added to a stirred solution of $\mathbf{3}$ (43.5 mg, 0.03 mmol, 1 equiv.) in THF ( $2 \mathrm{~mL})$ under 1 atm of $\mathrm{N}_{2}$ or Ar at RT. After stirring for $4 \mathrm{~h}$, the mixture was filtered through a sintered glass funnel and the volatiles were removed in vacuo. The residues were extracted with toluene and filtered, then the filtrate was concentrated to $0.5 \mathrm{~mL}$. After the concentrated solution was placed at $-30{ }^{\circ} \mathrm{C}$ for $24 \mathrm{~h}$, red-brown crystals of 4 suitable for X-ray diffraction were obtained (29.4 mg, 71\%). ${ }^{1} \mathrm{H}$ NMR $($ THF-d $8,400 \mathrm{MHz}, \mathrm{ppm}) \delta 74.77$ (s, 1H), 58.56 (s, 1H), $58.10(\mathrm{~s}, 6 \mathrm{H}), 52.06(\mathrm{~s}, 1 \mathrm{H}), 50.06(\mathrm{~s}, 1 \mathrm{H}), 40.80(\mathrm{~s}, 1 \mathrm{H}), 31.76(\mathrm{~s}, 1 \mathrm{H}), 14.45(\mathrm{~s}, 1 \mathrm{H}), 14.03$ (s, 1H), $13.85(\mathrm{~s}, 1 \mathrm{H}), 13.59(\mathrm{~s}, 6 \mathrm{H}), 11.24(\mathrm{~s}, 6 \mathrm{H}), 10.78(\mathrm{~s}, 1 \mathrm{H}), 0.42(\mathrm{~s}, 6 \mathrm{H}),-0.56(\mathrm{~s}, 6 \mathrm{H}),-1.84$ 
(s, 1H), -8.46 (s, 1H), -9.37 (s, 1H), -13.77 (s, 1H), -15.59 (s, 6H), -18.24 (s, 1H), -21.72 (s, 1H), $21.93(\mathrm{~s}, 6 \mathrm{H}),-22.43(\mathrm{~s}, 6 \mathrm{H}),-30.82(\mathrm{~s}, 1 \mathrm{H}),-37.18(\mathrm{~s}, 1 \mathrm{H}),-39.29(\mathrm{~s}, 1 \mathrm{H}),-39.33(\mathrm{~s}, 1 \mathrm{H}),,-53.94$ (s, 6H), -55.18 (s, 1H), -67.60 (s, 1H), -75.35 (s, 1H), -80.88 (s, 1H). ${ }^{31} \mathrm{P}\left\{{ }^{1} \mathrm{H}\right\}$ NMR (THF-d 8,162 MHz, ppm) $\delta 732.4$ (br), 64.2 (s). ${ }^{13} \mathrm{C}$ NMR spectrum could not be obtained due to its low solubility. Anal. Calcd. for $\mathrm{C}_{34} \mathrm{H}_{78} \mathrm{Cl}_{3} \mathrm{~N}_{6} \mathrm{P}_{4} \mathrm{RhU}_{2}$ : C 29.59; H 5.70; N 6.09. Found: C 29.90; H 5.75; N 6.13.

Synthesis of $\quad\left[\left\{\mathrm{U}_{2}\left[\mathrm{~N}\left(\mathrm{CH}_{3}\right)\left(\mathrm{CH}_{2} \mathrm{CH}_{2} \mathrm{NP}^{i} \mathrm{Pr}_{2}\right)_{2}\right]_{2}(\mathrm{Rh})(\mu-\mathrm{N})\right\}_{2}\right] \quad$ (5) and $\left[\left\{\mathrm{U}_{2}\left[\mathbf{N}\left(\mathrm{CH}_{3}\right)\left(\mathrm{CH}_{2} \mathrm{CH}_{2} \mathrm{NP}^{i} \mathrm{Pr}_{2}\right)_{2}\right]_{2}(\mathrm{Rh})\left(\mu-{ }^{15} \mathrm{~N}\right)\right\}_{2}\right]\left(5^{15} \mathrm{~N}\right)$

\section{Method A}

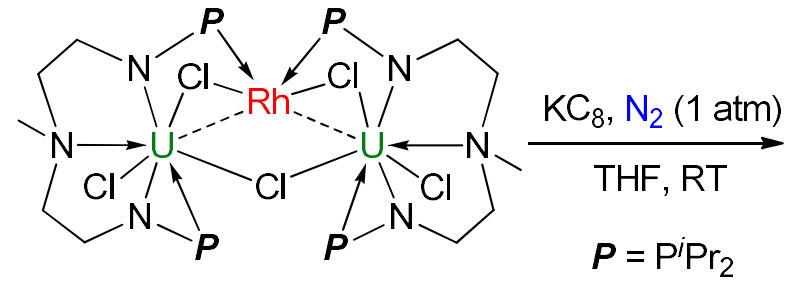

3

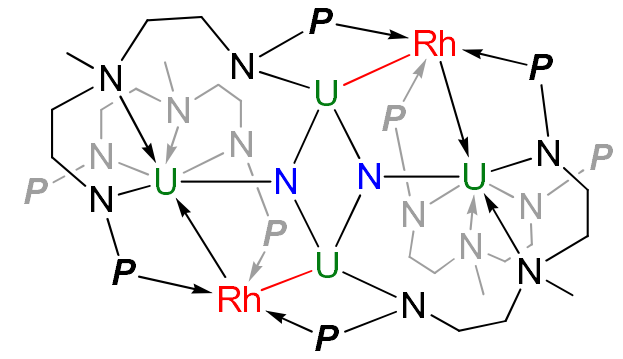

5

A slurry of $\mathrm{KC}_{8}(20.3 \mathrm{mg}, 0.15 \mathrm{mmol}, 5$ equiv. $)$ in $\mathrm{THF}(1 \mathrm{~mL})$ was added to a stirred solution of $\mathbf{3}$

(43.5 mg, $0.03 \mathrm{mmol}, 1$ equiv.) in THF $(1 \mathrm{~mL})$ under $1 \mathrm{~atm}$ of $\mathrm{N}_{2}$ at RT. After stirring for $6 \mathrm{~h}$, the mixture was filtered through a sintered glass funnel and the filtrate was concentrated to $0.5 \mathrm{~mL}$. After the concentrated solution was placed at $-30{ }^{\circ} \mathrm{C}$ for $24 \mathrm{~h}$, dark brown crystals of 5 suitable for X-ray diffraction were obtained (14.3 mg, $37 \%$ ). ${ }^{-15} \mathbf{N}$ was prepared by exposing 3 to ${ }^{15} \mathrm{~N}_{2}$. A mixture of 3 (43.5 mg, $0.03 \mathrm{mmol}, 1$ equiv.) and $\mathrm{KC}_{8}(20.3 \mathrm{mg}, 0.15 \mathrm{mmol}, 5$ equiv.) in THF ( $2 \mathrm{~mL})$ was freeze-pump-thaw degassed three times and exposed to an atmosphere of ${ }^{15} \mathrm{~N}_{2}$ (1 atm), following the same workup as 5 to afford $\mathbf{5 - 1 5}^{15} \mathbf{N} .{ }^{1} \mathrm{H}$ NMR (THF-d $\left.8,400 \mathrm{MHz}, \mathrm{ppm}\right) \delta 91.21(\mathrm{~s}, 1 \mathrm{H})$, $80.89(\mathrm{~s}, 1 \mathrm{H}), 64.26(\mathrm{~s}, 1 \mathrm{H}), 60.72(\mathrm{~s}, 1 \mathrm{H}), 54.20(\mathrm{~s}, 1 \mathrm{H}), 53.90(\mathrm{~s}, 3 \mathrm{H}), 51.37$ (s, 1H), $44.16(\mathrm{~s}, 1 \mathrm{H})$, 
$37.23(\mathrm{~s}, 1 \mathrm{H}), 34.87$ (s, 3H), $27.76(\mathrm{~s}, 1 \mathrm{H}), 27.45(\mathrm{~s}, 1 \mathrm{H}), 18.26(\mathrm{~s}, 1 \mathrm{H}), 17.62(\mathrm{~s}, 1 \mathrm{H}), 17.34(\mathrm{~s}, 3 \mathrm{H})$, $16.98(\mathrm{~s}, 3 \mathrm{H}), 13.41(\mathrm{~s}, 1 \mathrm{H}), 8.05,(\mathrm{~s}, 3 \mathrm{H})-3.55(\mathrm{~s}, 2 \mathrm{H}),-4.01(\mathrm{~s}, 6 \mathrm{H}),-4.64(\mathrm{~s}, 6 \mathrm{H}),-4.99(\mathrm{~s}, 6 \mathrm{H}),-$ $6.17(\mathrm{~s}, 1 \mathrm{H}),-8.77$ (s, 3H), -11.19 (s, 3H), -15.45 (s, 1H), -18.75, (s, 3H) -20.61 (s, 3H), -21.49 (s, 1H), $-32.05(\mathrm{~s}, 3 \mathrm{H}),-36.14(\mathrm{~s}, 3 \mathrm{H}),-37.91(\mathrm{~s}, 1 \mathrm{H}),-43.16(\mathrm{~s}, 1 \mathrm{H}),-48.90(\mathrm{~s}, 1 \mathrm{H}),-53.16(\mathrm{~s}, 1 \mathrm{H}),-$ $81.48(\mathrm{~s}, 3 \mathrm{H}),-112.39(\mathrm{~s}, 2 \mathrm{H}) .{ }^{13} \mathrm{C}$ NMR spectrum could not be obtained due to its low solubility after recrystallization from THF. ${ }^{31} \mathrm{P}\left\{{ }^{1} \mathrm{H}\right\}$ NMR $\left(\mathrm{THF}-\mathrm{d}_{8}, 162 \mathrm{MHz}, \mathrm{ppm}\right) \delta$ 64.2. The signal of the $\mathrm{P}$ atom which bonded directly to the uranium center was unobserved ranging from +1000 to -1000 ppm. Anal. Calcd. for $\mathrm{C}_{68} \mathrm{H}_{156} \mathrm{~N}_{14} \mathrm{P}_{8} \mathrm{Rh}_{2} \mathrm{U}_{4} \cdot 2 \mathrm{THF}$ : C 33.56; H 6.37; N 7.21. Found: C 33.54; H 6.39; N 7.09.

\section{Method B}

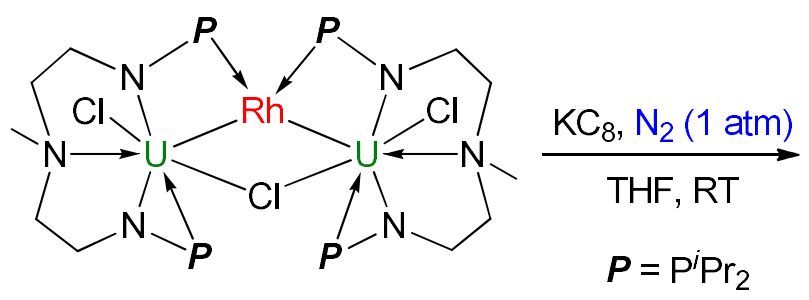

4

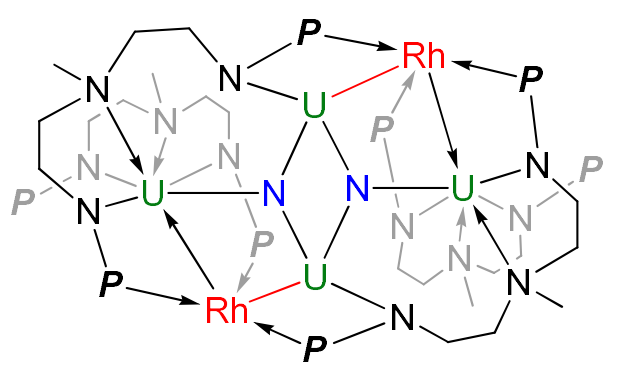

5

A slurry of $\mathrm{KC}_{8}(12.2 \mathrm{mg}, 0.09 \mathrm{mmol}, 3$ equiv.) in THF was added to a stirred solution of 4 (41.4 $\mathrm{mg}, 0.03 \mathrm{mmol}, 1$ equiv.) in THF ( $2 \mathrm{~mL})$ under $1 \mathrm{~atm}$ of $\mathrm{N}_{2}$ at RT. After stirring for $2 \mathrm{~h}$, the mixture was filtered through a sintered glass funnel and the filtrate was concentrated to $0.5 \mathrm{~mL}$. After the concentrated solution was held at $-30^{\circ} \mathrm{C}$ for $24 \mathrm{~h}$, dark brown crystals of 5 suitable for X-ray diffraction were obtained (15.1 mg, 39\%).

\section{Procedure for ammonia formation from complex 5 and $5-{ }^{15} \mathrm{~N}$}

An excess of PyHCl (26.6 mg, $0.23 \mathrm{mmol}, 50$ equiv.) was added to a brown solution of 5 (12 $\mathrm{mg}$, 
$0.0046 \mathrm{mmol}, 1$ equiv. $)$ in THF ( $1 \mathrm{ml})$. The mixture was stirred at RT for overnight, affording a paleyellow solution with a white precipitate. The supernatant was removed and the solid was washed three times with $1.0 \mathrm{ml}$ of THF. The solid was dried under vacuum. ${ }^{1} \mathrm{H}$ NMR analysis shows two products, ammonium chloride $\left(\mathrm{NH}_{4} \mathrm{Cl}\right)$ and the protonated 1 . The amount of ammonia was evaluated by quantitative ${ }^{1} \mathrm{H}$ NMR with dibromomethane as an internal standard ( $82 \%$ yield of ammonia). ${ }^{15} \mathrm{NH}_{4} \mathrm{Cl}$ was prepared from ${ }^{5-15} \mathbf{N}$ following the same procedure. ${ }^{1} \mathrm{H}$ NMR of $\mathrm{NH}_{4} \mathrm{Cl}$ (DMSO- $\mathrm{d}_{6}$, $400 \mathrm{MHz}, \mathrm{ppm}): \delta=7.32$ (t, $J=52 \mathrm{~Hz}$ ); ${ }^{1} \mathrm{H}$ NMR of ${ }^{15} \mathrm{NH}_{4} \mathrm{Cl}$ (DMSO-d $\left.6,400 \mathrm{MHz}, \mathrm{ppm}\right): \delta=7.32$ $(\mathrm{d}, J=72 \mathrm{~Hz})$. 


\section{Supporting Figures}

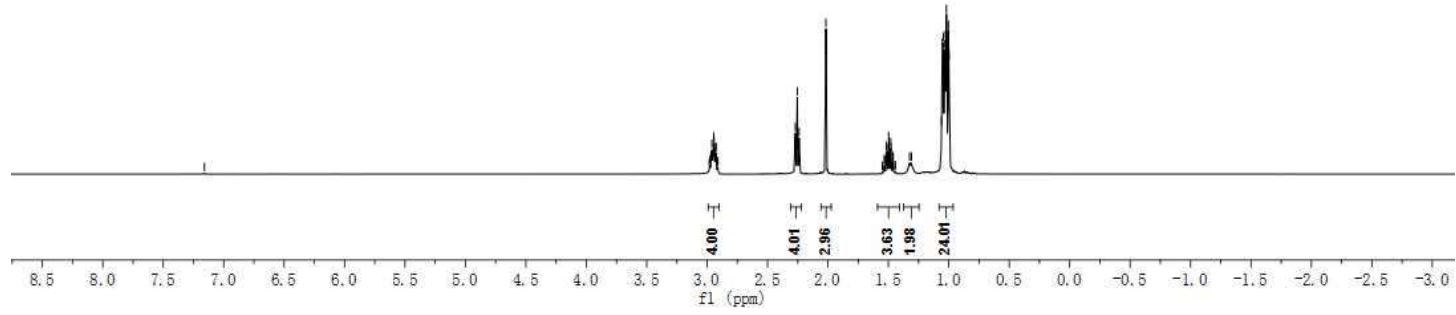

Figure S1. The ${ }^{1} \mathrm{H}$ NMR (400 MHz) spectrum of compound $\mathbf{1}$ in $\mathrm{C}_{6} \mathrm{D}_{6}$.

\section{หู.}
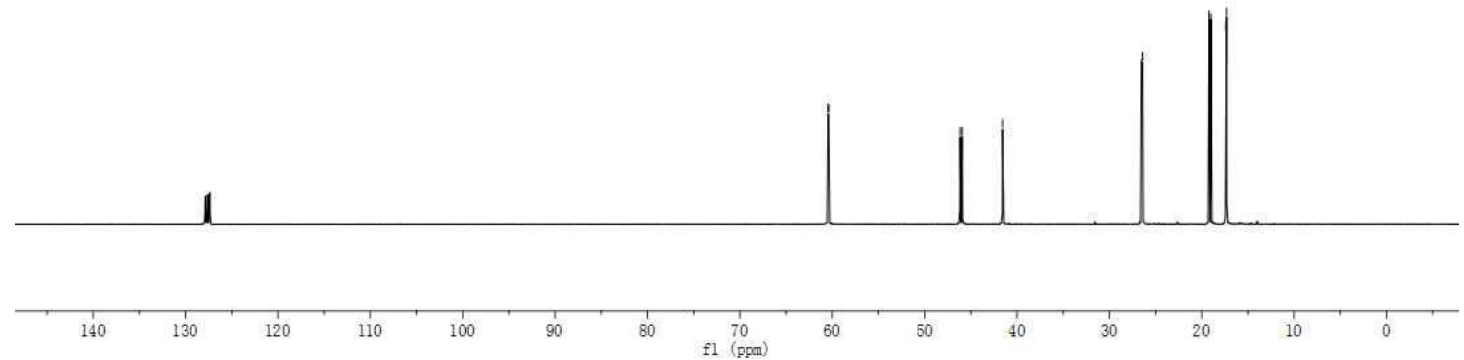

Figure S2. The ${ }^{13} \mathrm{C}\left\{{ }^{1} \mathrm{H}\right\}$ NMR (101 MHz) spectrum of compound $\mathbf{1}$ in $\mathrm{C}_{6} \mathrm{D}_{6}$. 


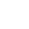

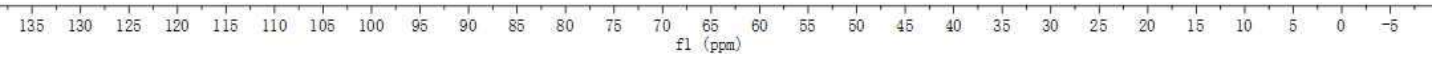

Figure S3. The ${ }^{31} \mathrm{P}\left\{{ }^{1} \mathrm{H}\right\}$ NMR $(162 \mathrm{MHz})$ spectrum of compound 1 in $\mathrm{C}_{6} \mathrm{D}_{6}$.
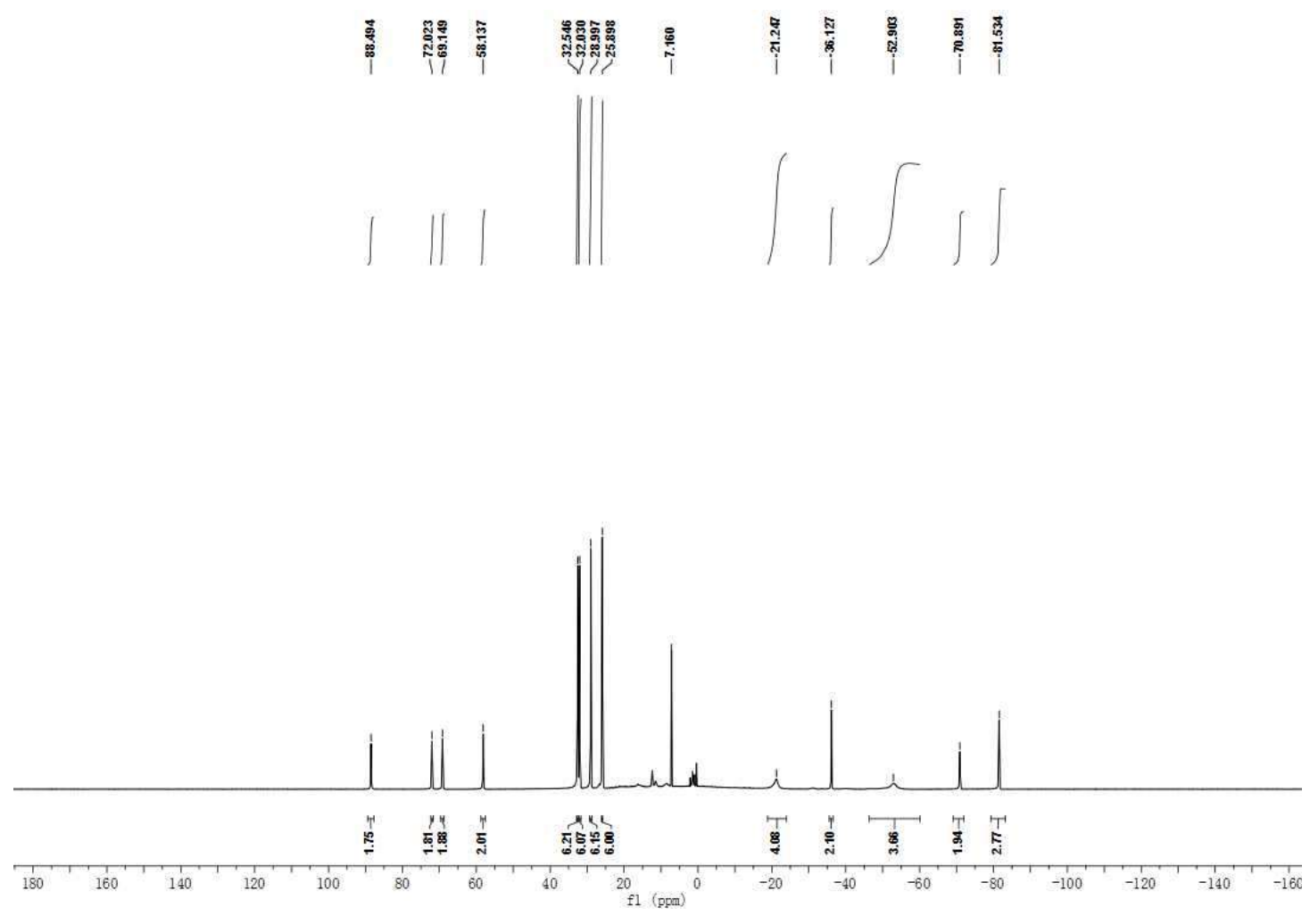

Figure S4. The ${ }^{1} \mathrm{H}$ NMR (400 MHz) spectrum of complex 2 in $\mathrm{C}_{6} \mathrm{D}_{6}$. 


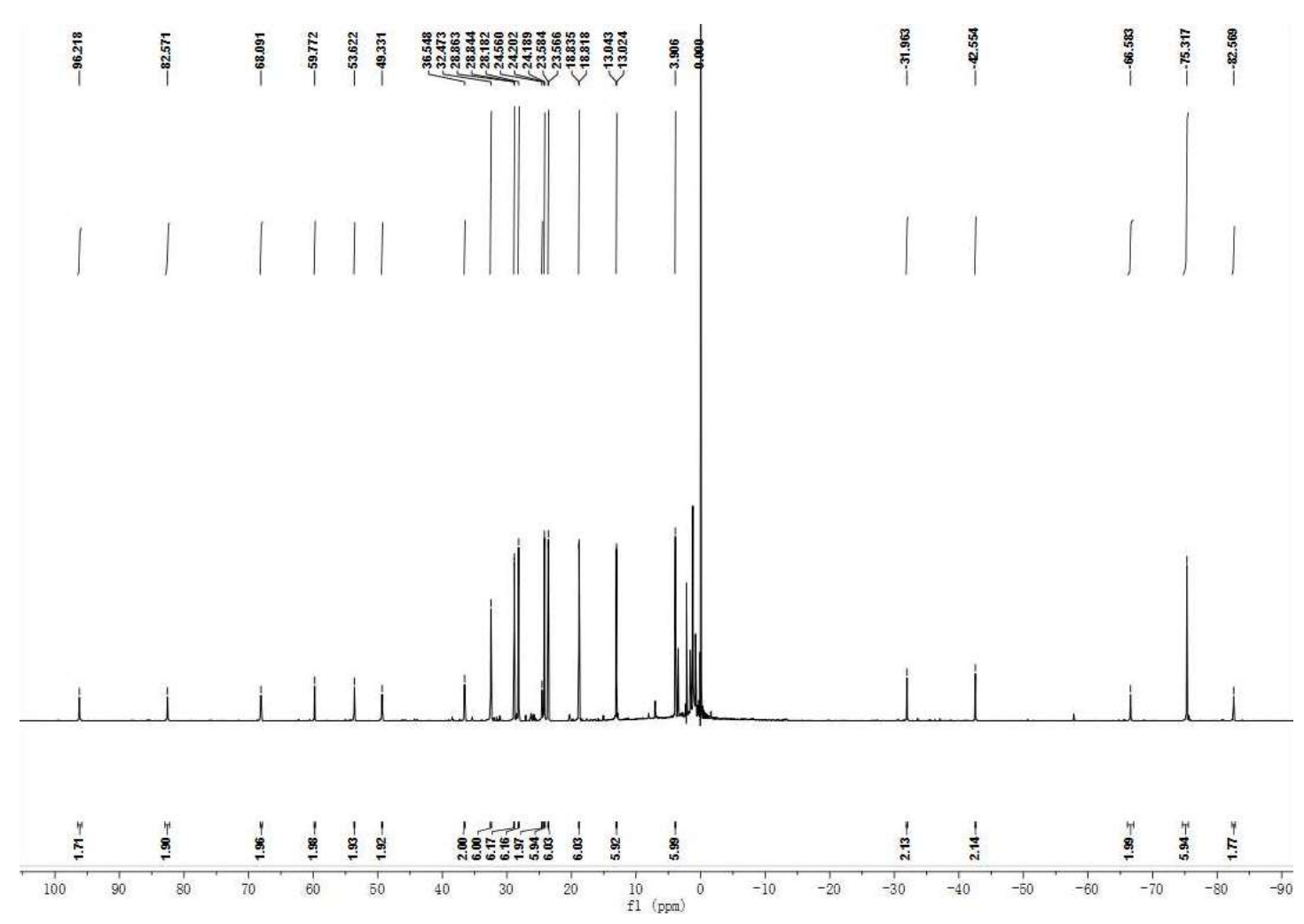

Figure S5. The ${ }^{1} \mathrm{H}$ NMR (400 MHz) spectrum of complex 3 in THF-d 8 .
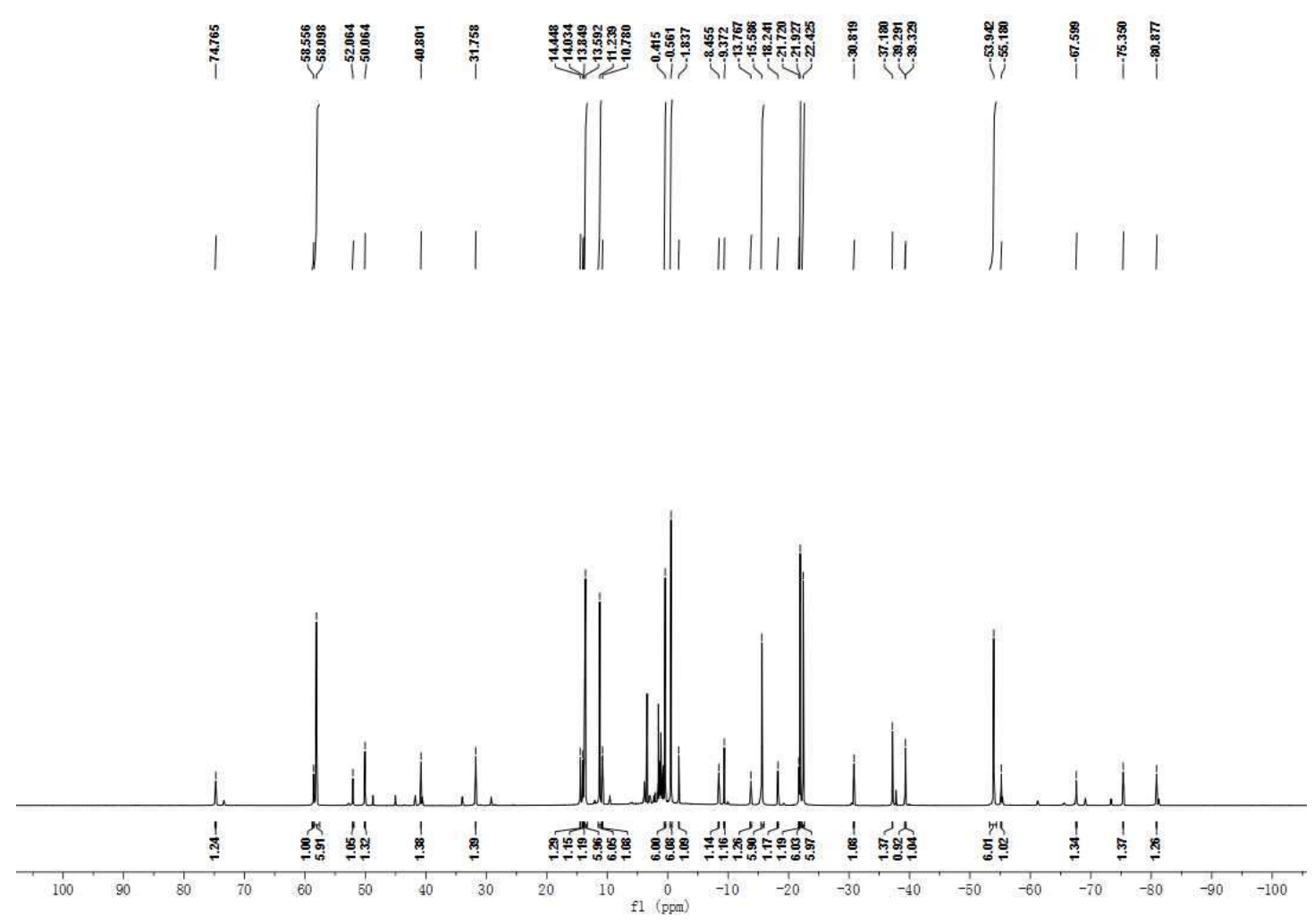

Figure S6. The ${ }^{1} \mathrm{H}$ NMR (400 MHz) spectrum of complex 4 in THF-d 8 . 


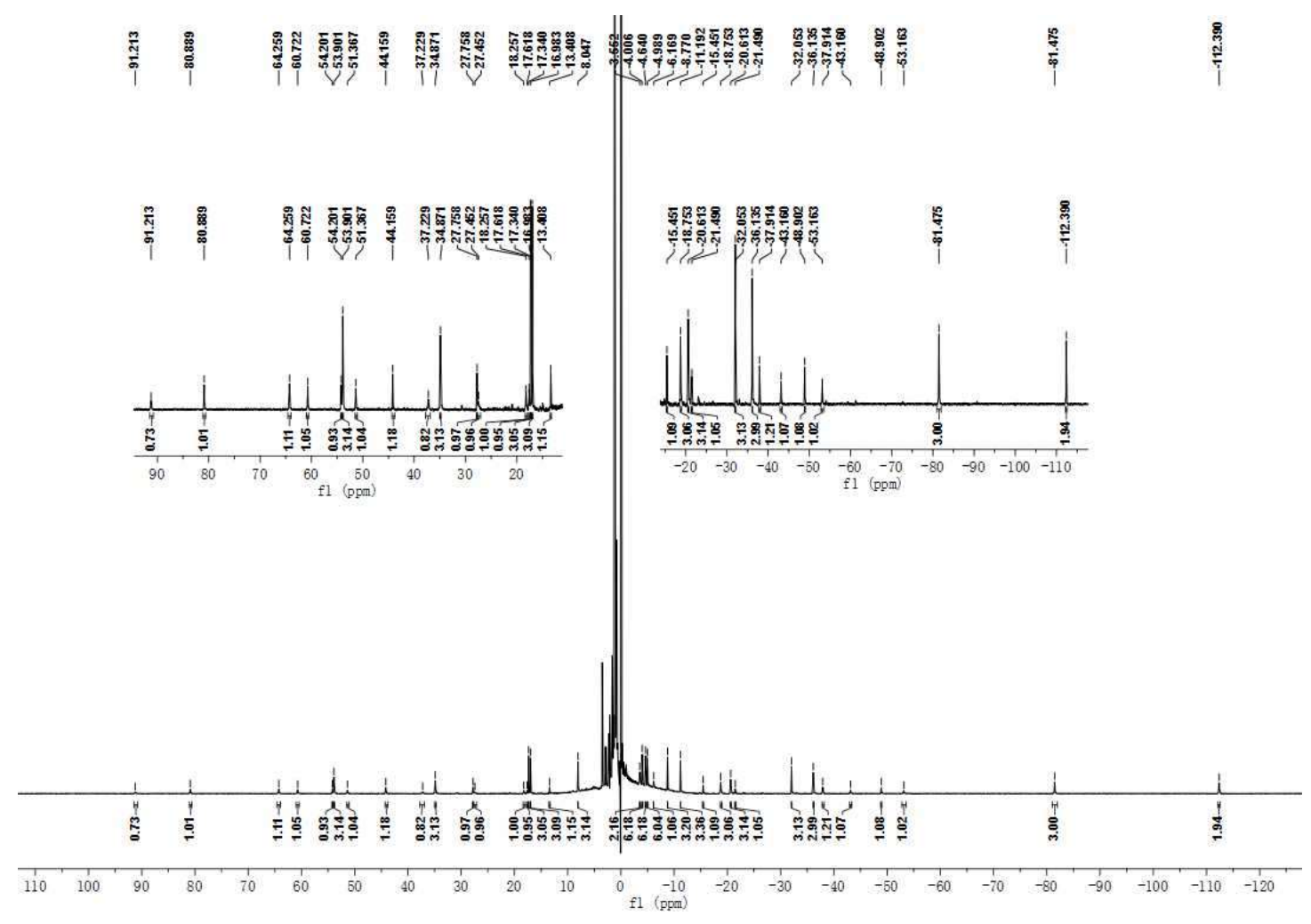

Figure S7. The ${ }^{1} \mathrm{H}$ NMR (400 MHz) spectrum of complex 5 in THF-d8.

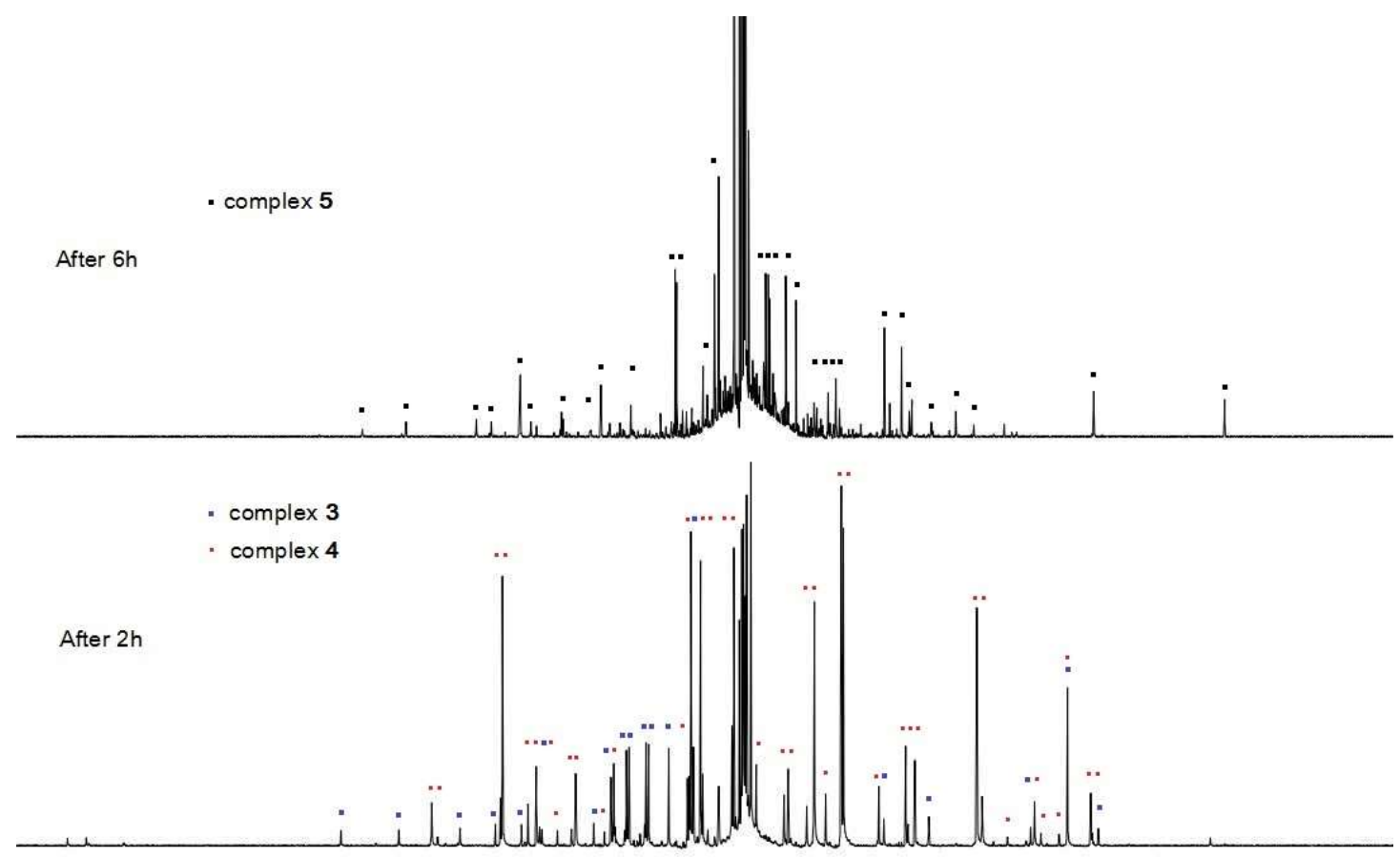

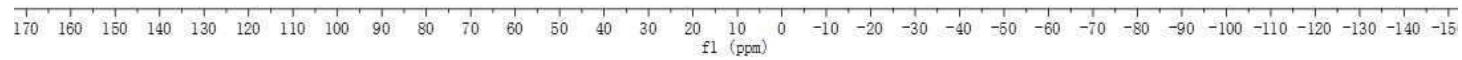

Figure S8. The in situ ${ }^{1} \mathrm{H}$ NMR ( $400 \mathrm{MHz}$ ) spectra for the reaction of complex 3 with $\mathrm{KC}_{8}$ (5 equiv.) at RT in THF- $\mathrm{d}_{8}$ in an NMR tube. 


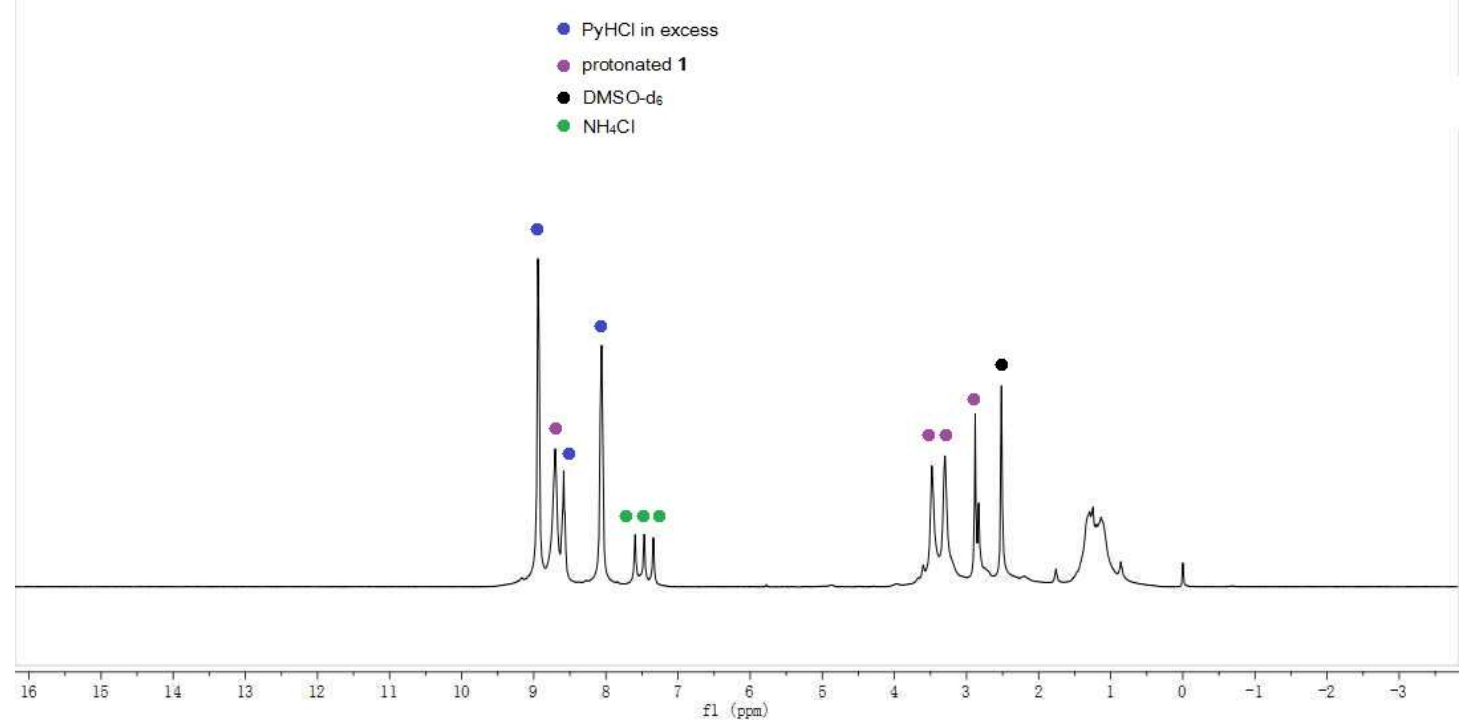

Figure S9. The ${ }^{1} \mathrm{H}$ NMR (400 MHz, DMSO- $\mathrm{d}_{6}$ ) spectrum of $\mathrm{NH}_{4} \mathrm{Cl}$ formed from the reaction of complex 5 with PyHCl.

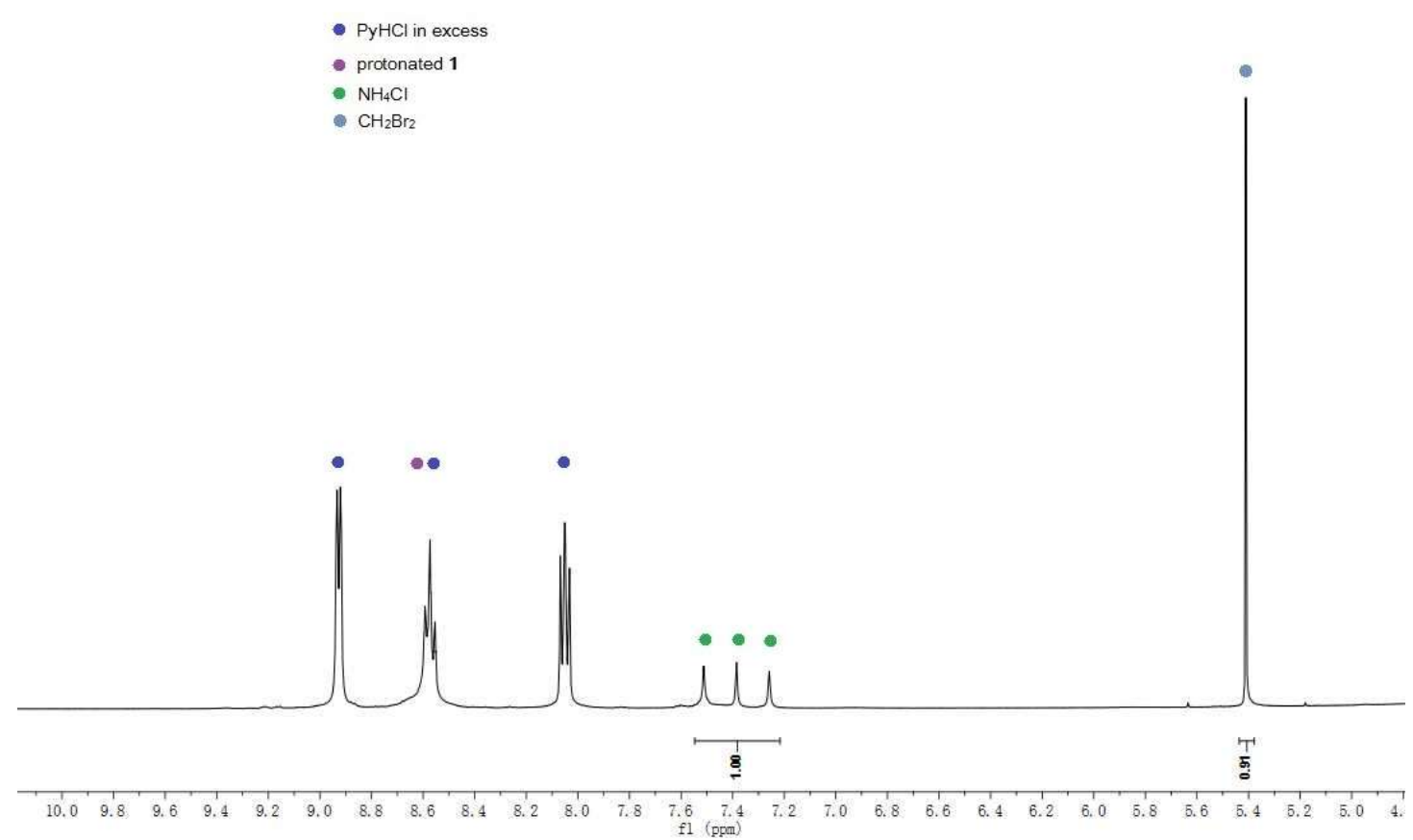

Figure S10. The ${ }^{1} \mathrm{H}$ NMR (400 MHz, DMSO- $\mathrm{d}_{6}$ ) spectrum of $\mathrm{NH}_{4} \mathrm{Cl}$ formed from the reaction of complex 5 with $\mathrm{PyHCl}$ (3 equiv. of dibromomethane was added for quantitative determination). 

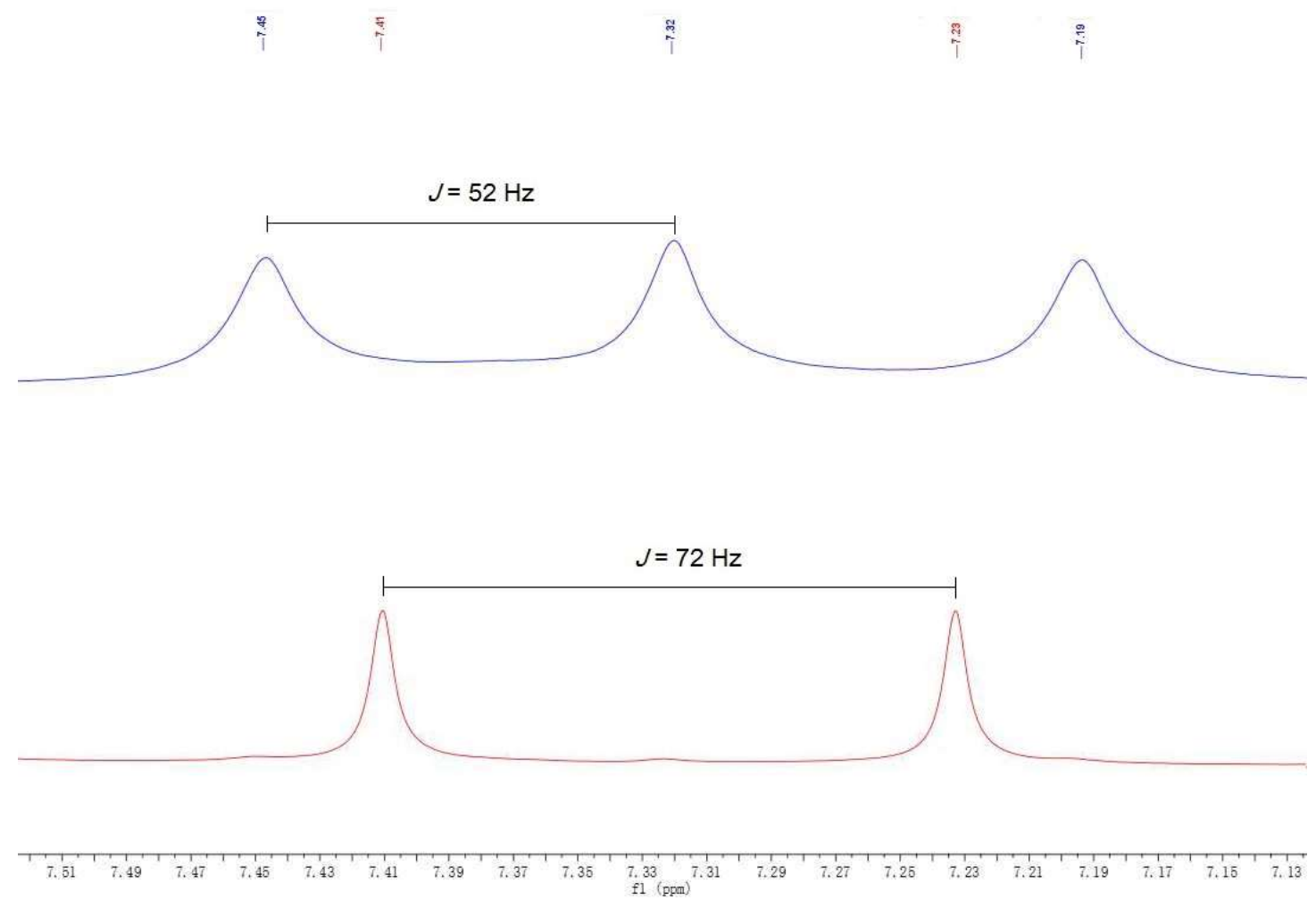

Figure S11. The ${ }^{1} \mathrm{H}$ NMR (400 MHz, DMSO-d $\mathrm{d}_{6}$ ) spectra of $\mathrm{NH}_{4} \mathrm{Cl}$ (blue) and ${ }^{15} \mathrm{NH}_{4} \mathrm{Cl}$ (red) formed by the reactions of complexes $\mathbf{5}$ and $\mathbf{5}-{ }^{15} \mathbf{N}$ with $\mathrm{PyHCl}$, respectively. 


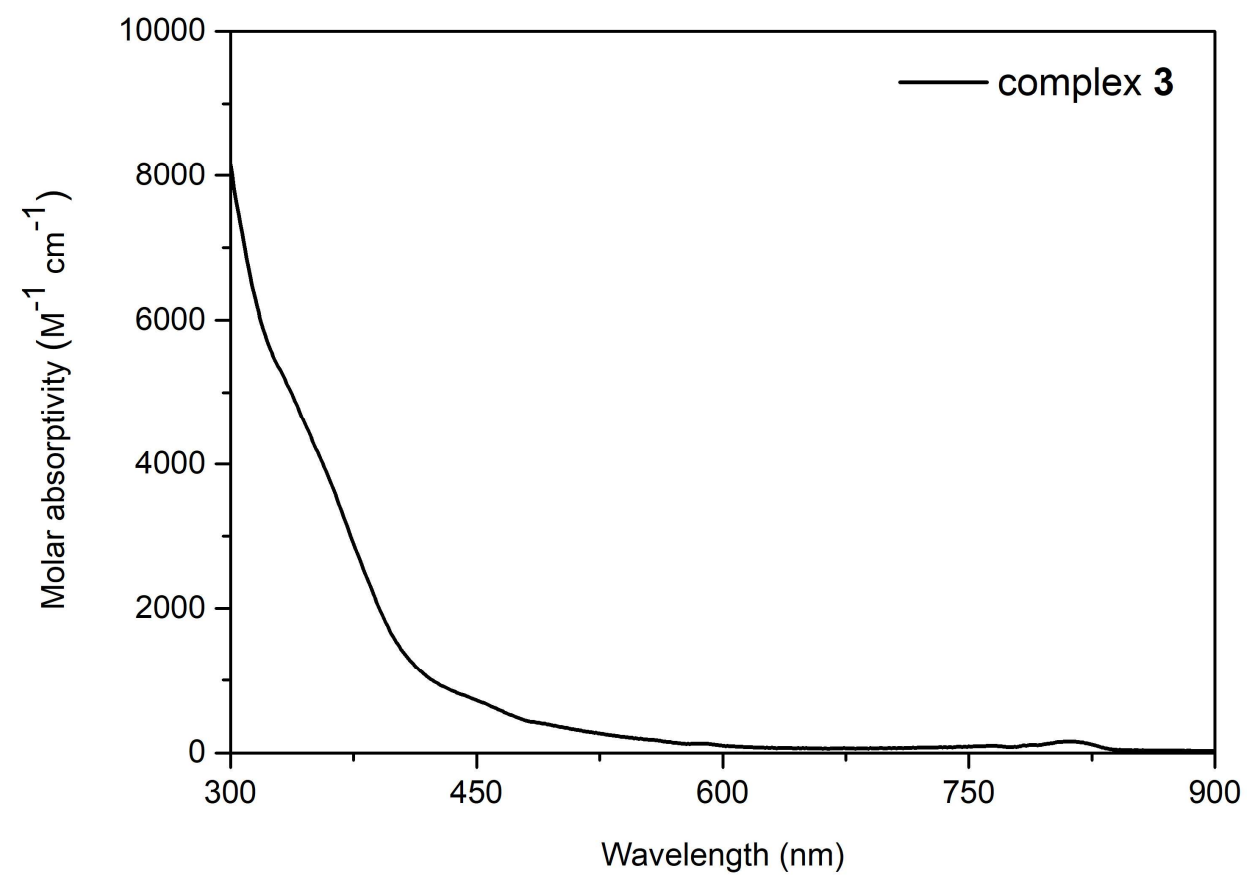

Figure S12. UV-visible absorption spectrum of complex 3 measured in THF at RT.

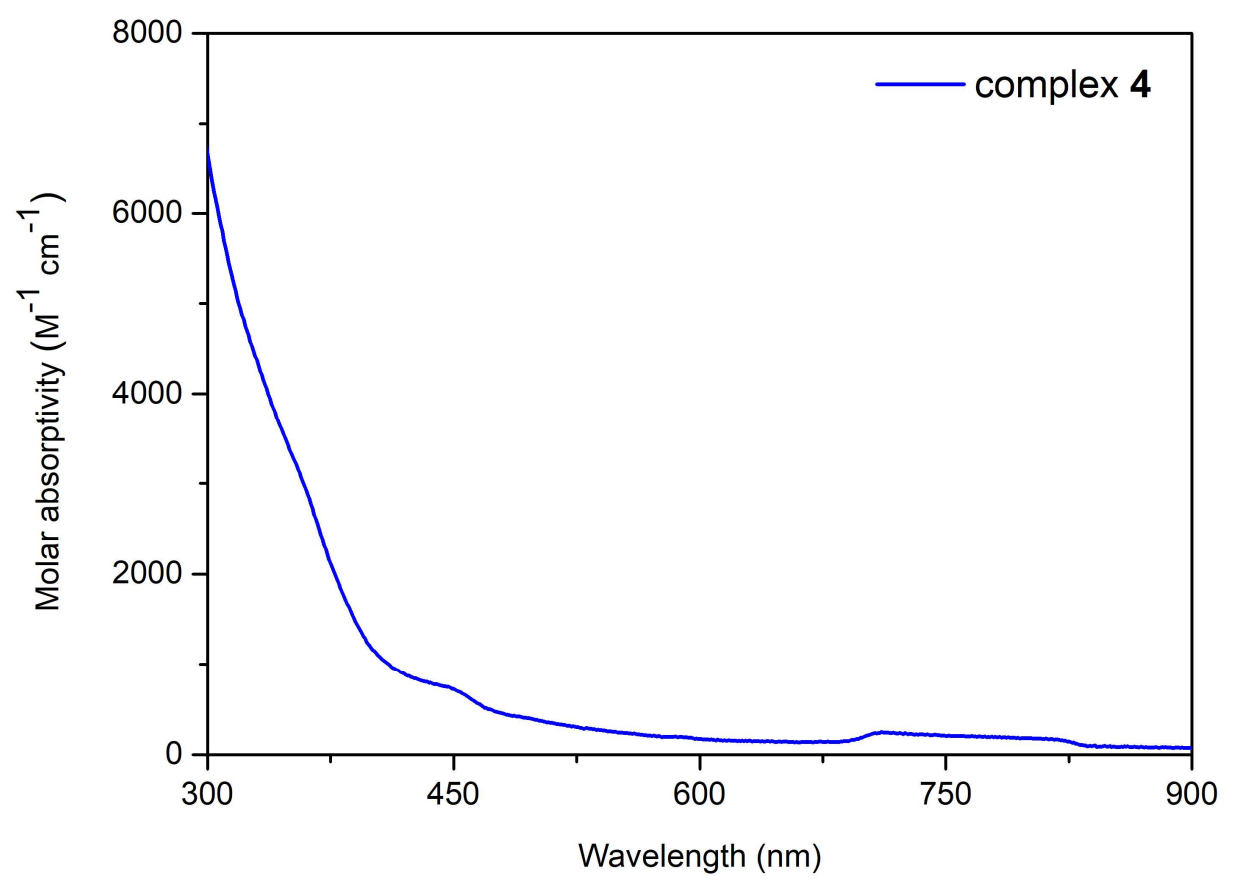

Figure S13. UV-visible absorption spectrum of complex 4 measured in THF at RT. 


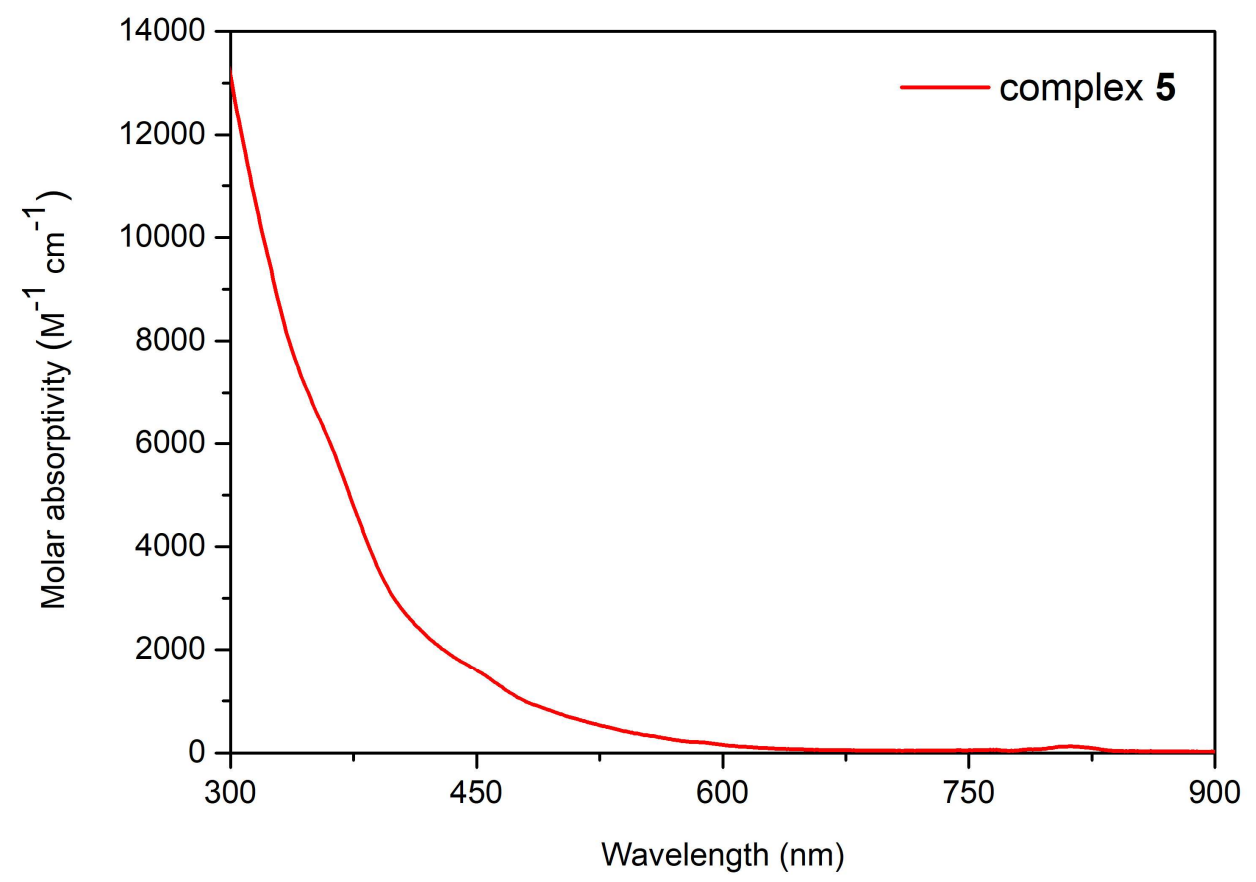

Figure S14. UV-visible absorption spectrum of complex $\mathbf{5}$ measured in THF at RT.

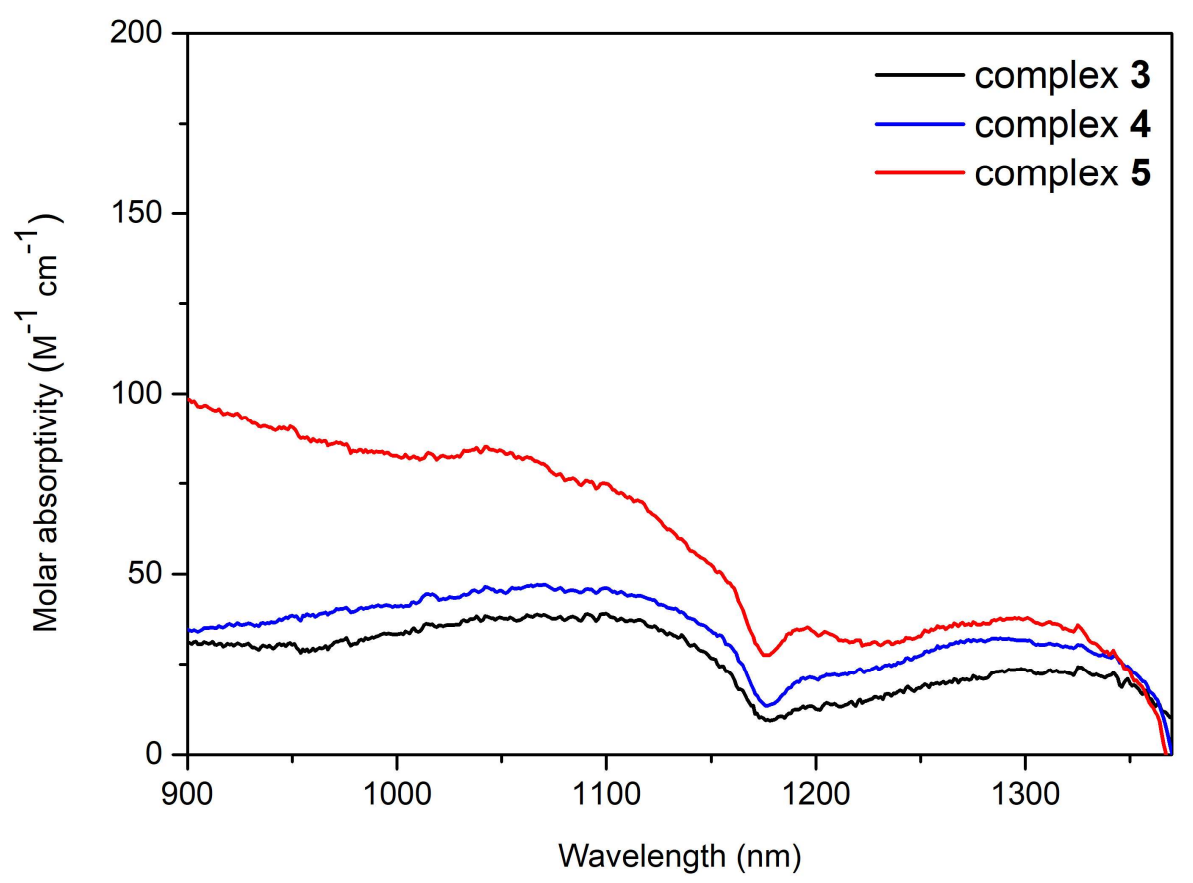

Figure S15. Near-infrared absorption spectra of complexes 3-5 in THF at RT. 
(a)

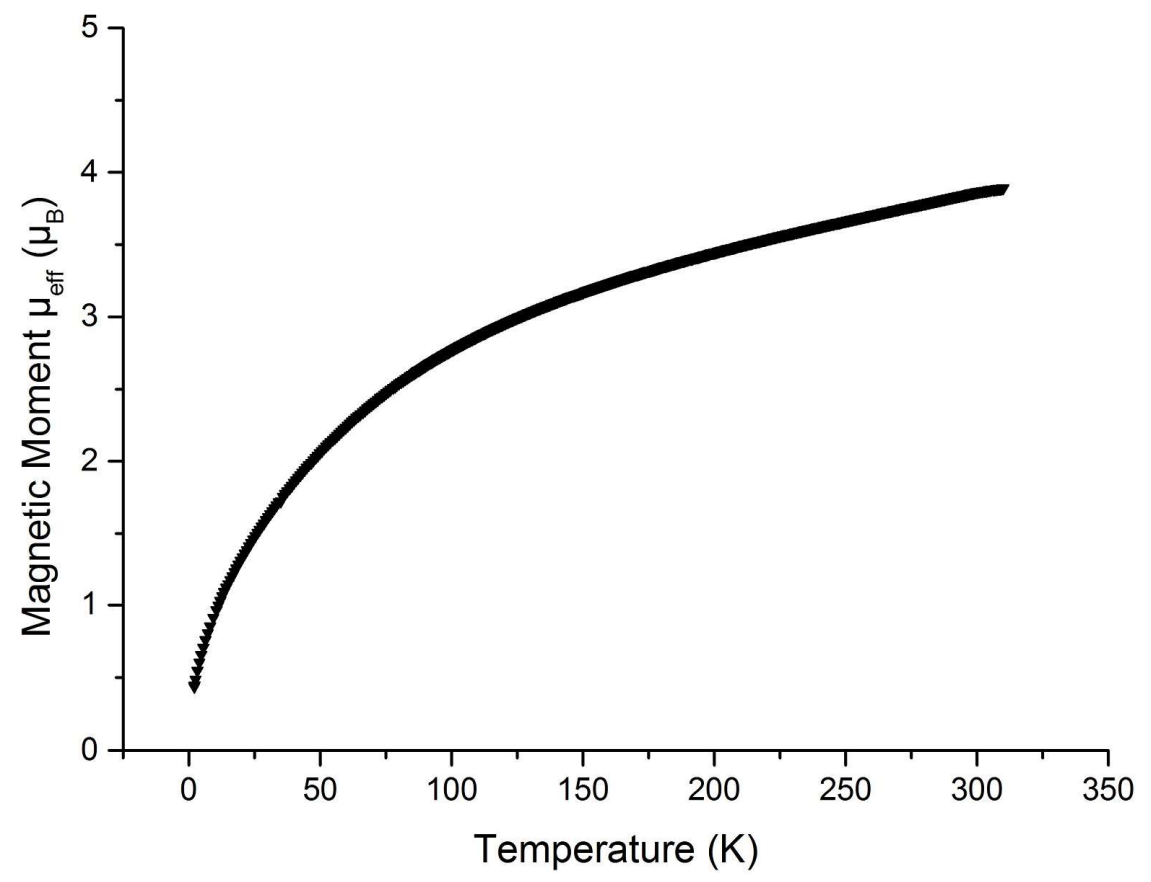

(b)

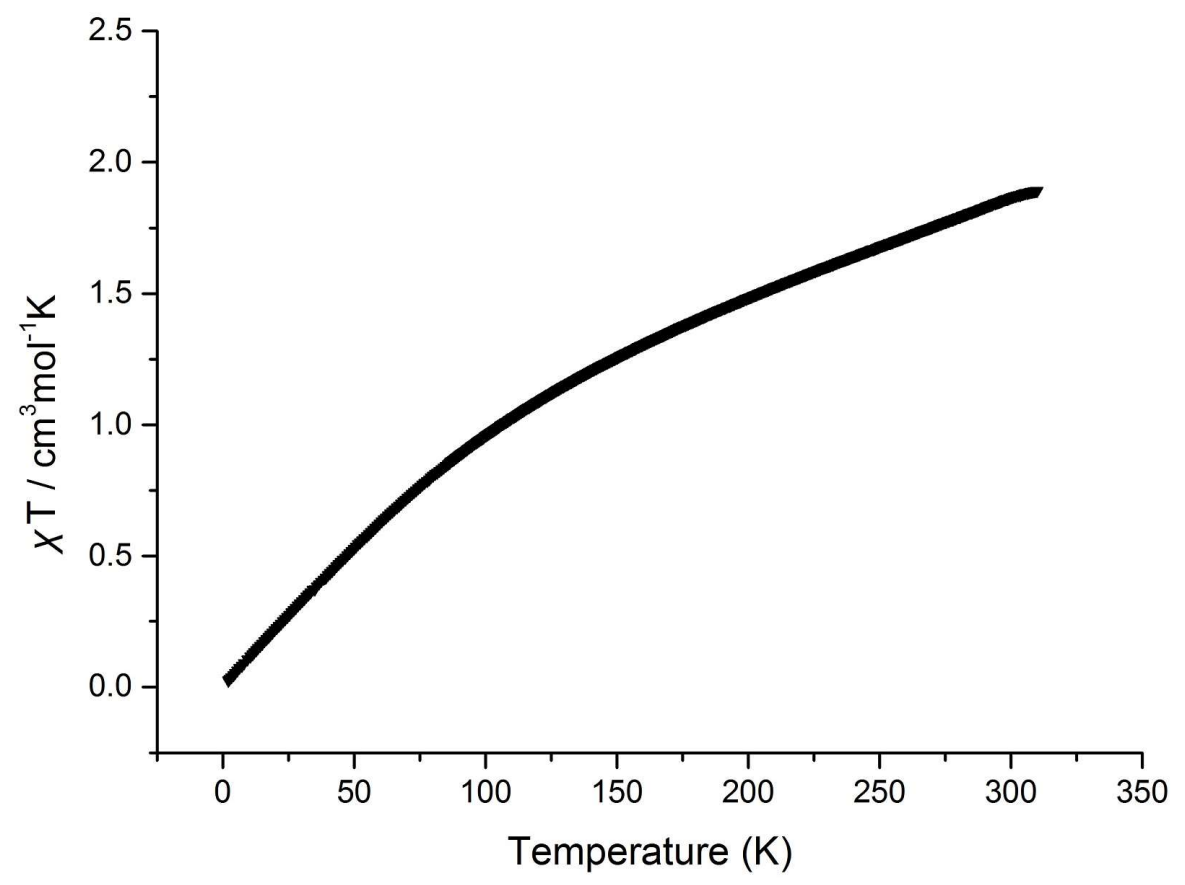

Figure S16. Variable-temperature magnetic data of complex 2 as $\mu_{\text {eff }}$ vs $\mathrm{T}(\mathrm{a}), \chi \mathrm{T}$ vs $\mathrm{T}(\mathrm{b})$. 
(a)

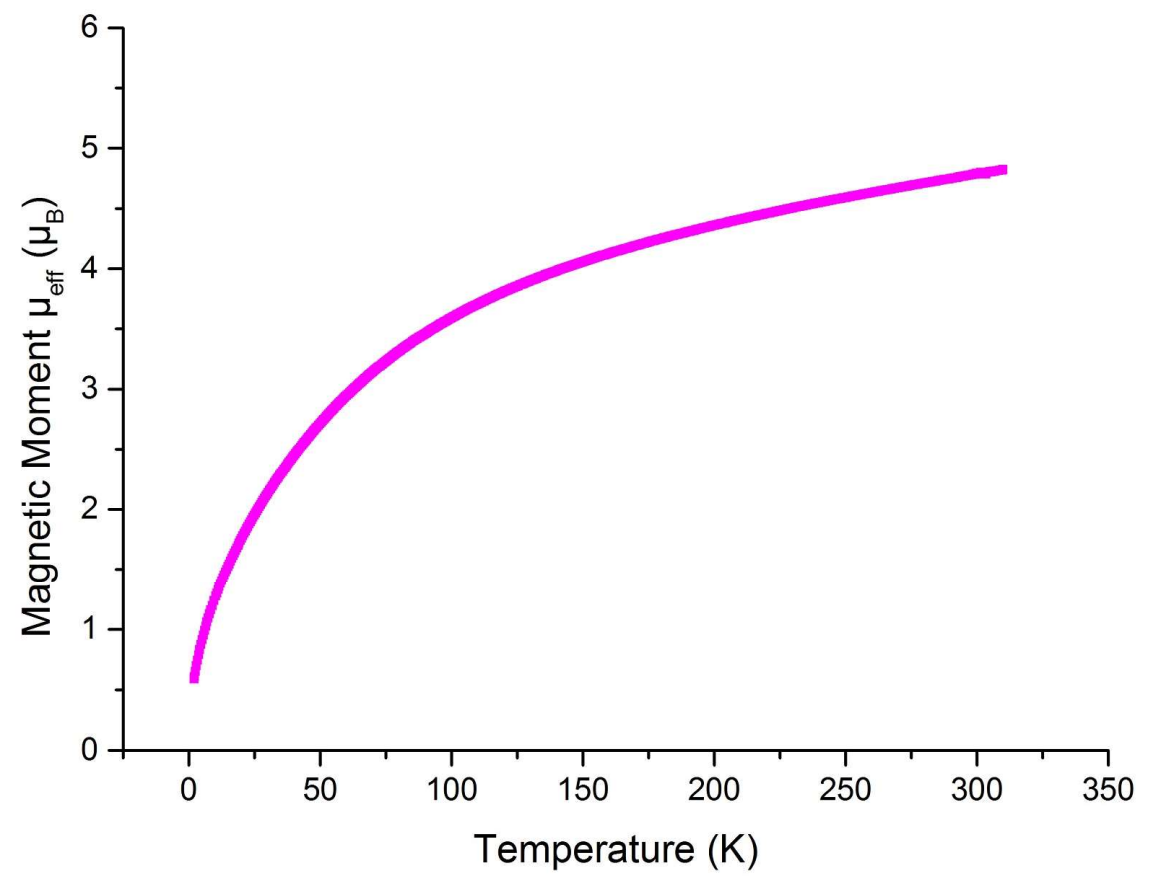

(b)

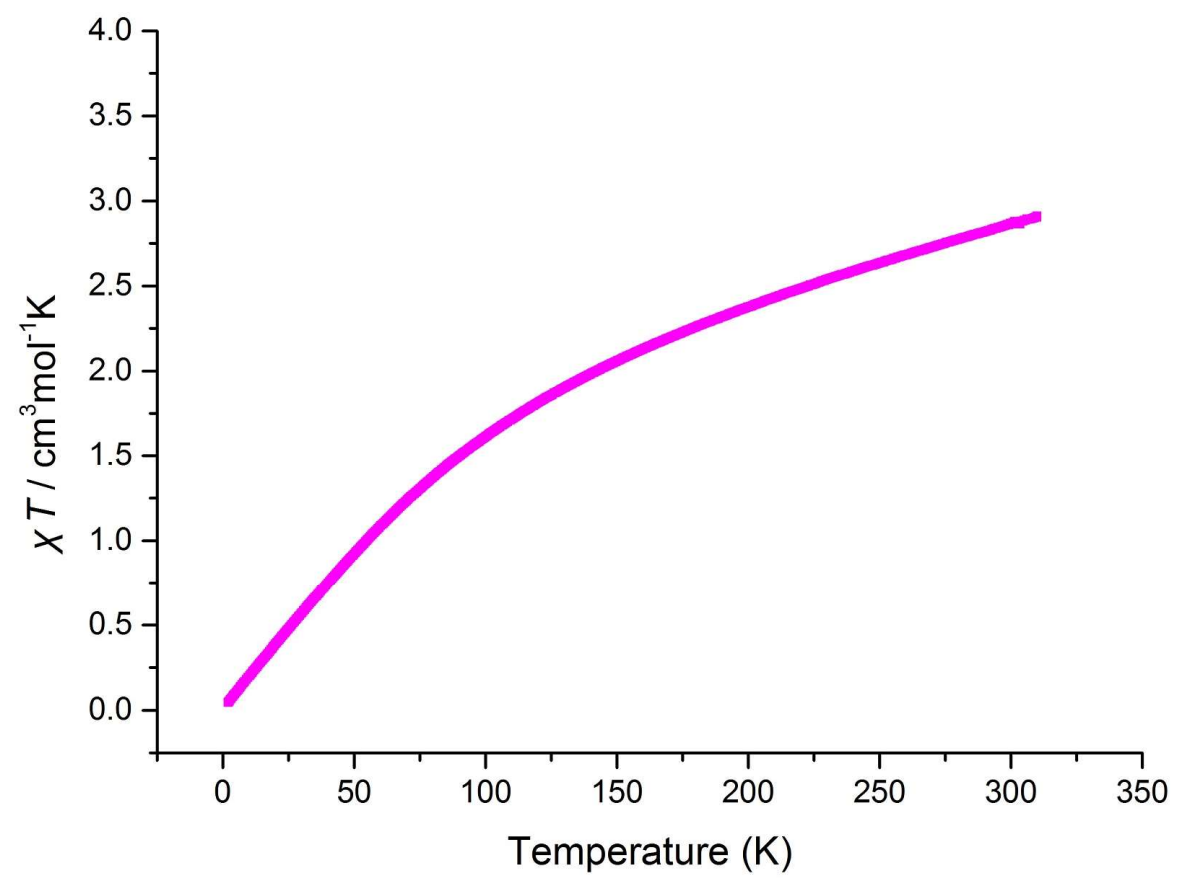

Figure S17. Variable-temperature magnetic data of complex $\mathbf{3}$ as $\mu_{\mathrm{eff}} \mathrm{vs} \mathrm{T}(\mathrm{a}), \chi \mathrm{T}$ vs $\mathrm{T}(\mathrm{b})$. 
(a)

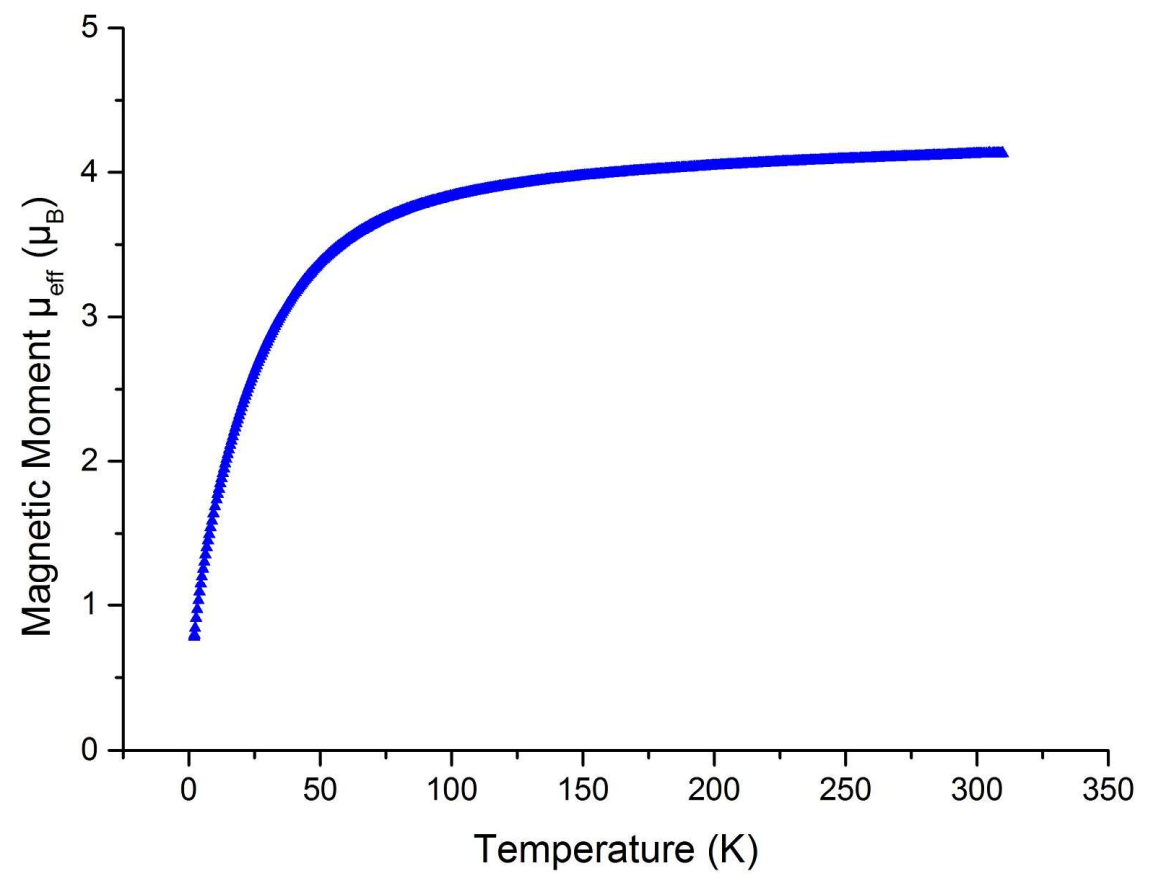

(b)

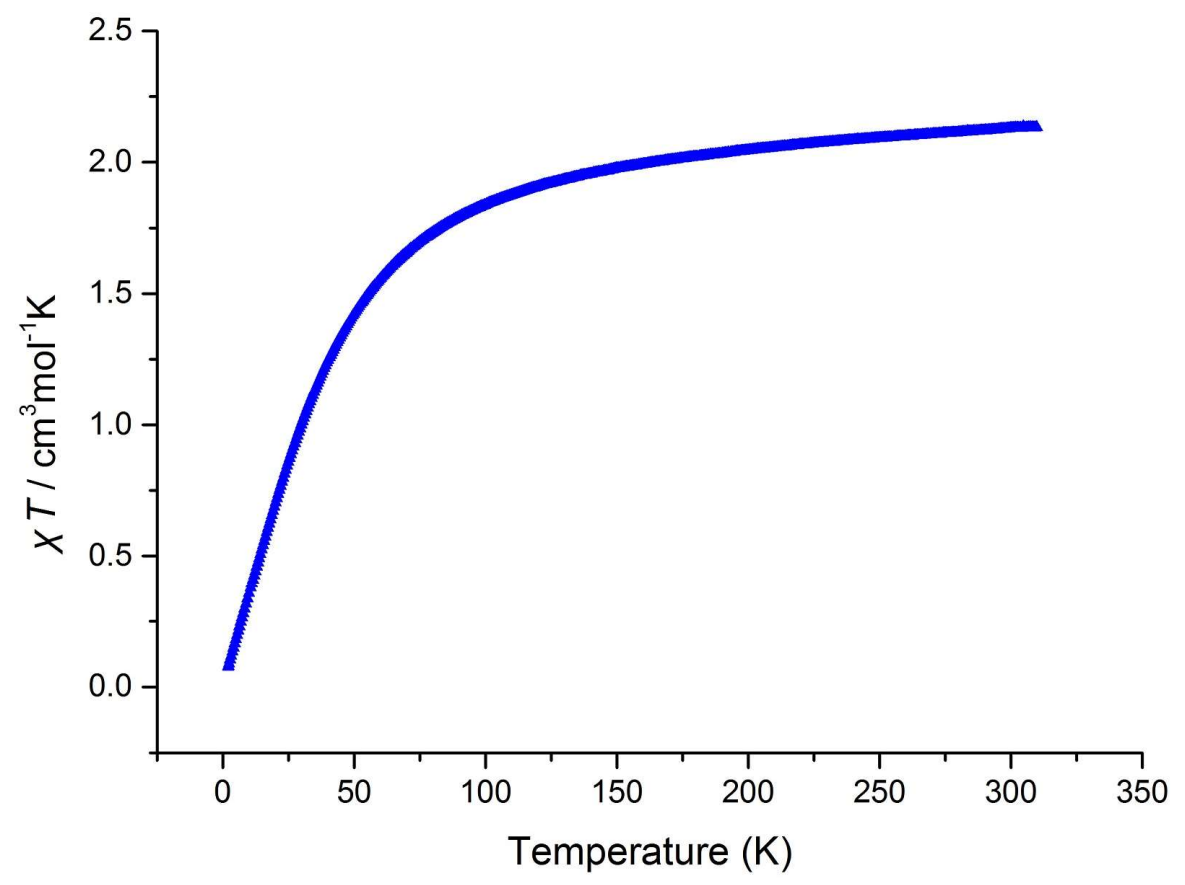

Figure S18. Variable-temperature magnetic data of complex 4 as $\mu_{\text {eff }}$ vs $\mathrm{T}(\mathrm{a}), \chi \mathrm{T}$ vs $\mathrm{T}$ (b). 
(a)

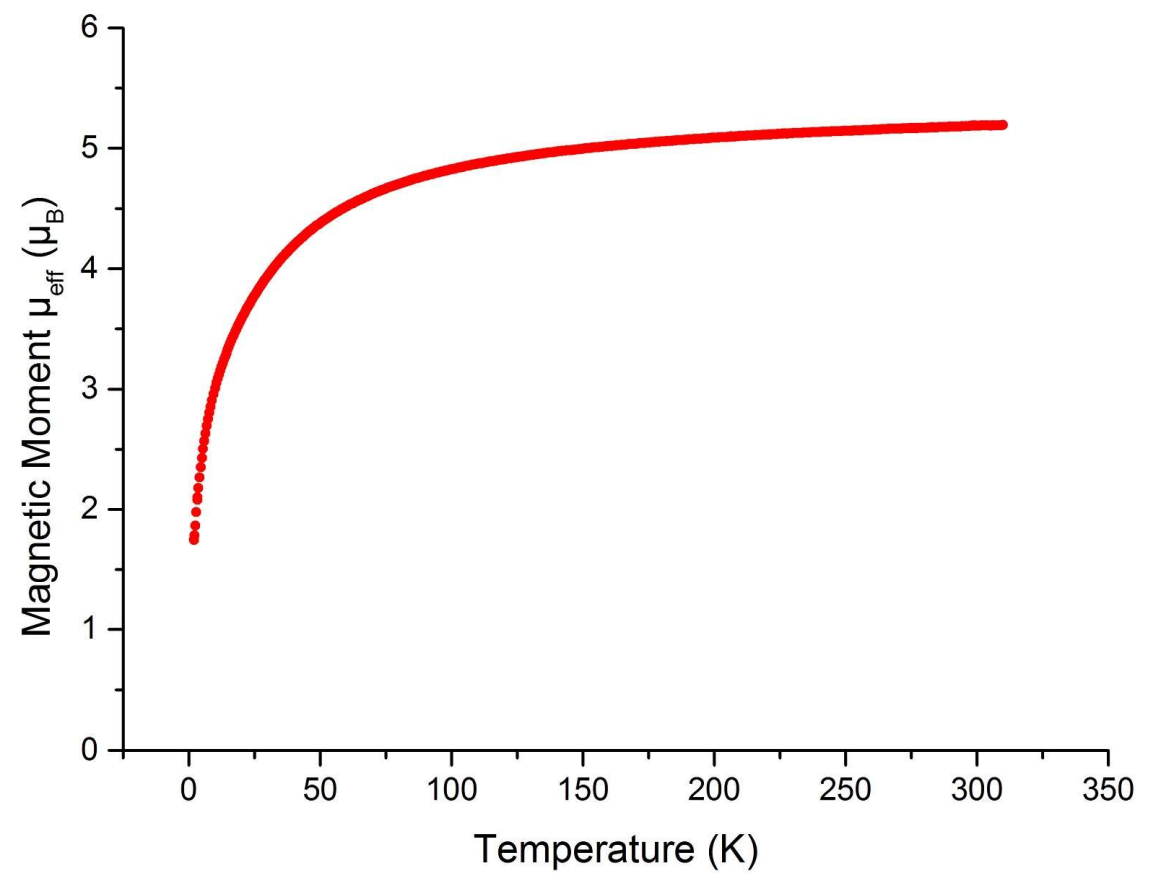

(b)

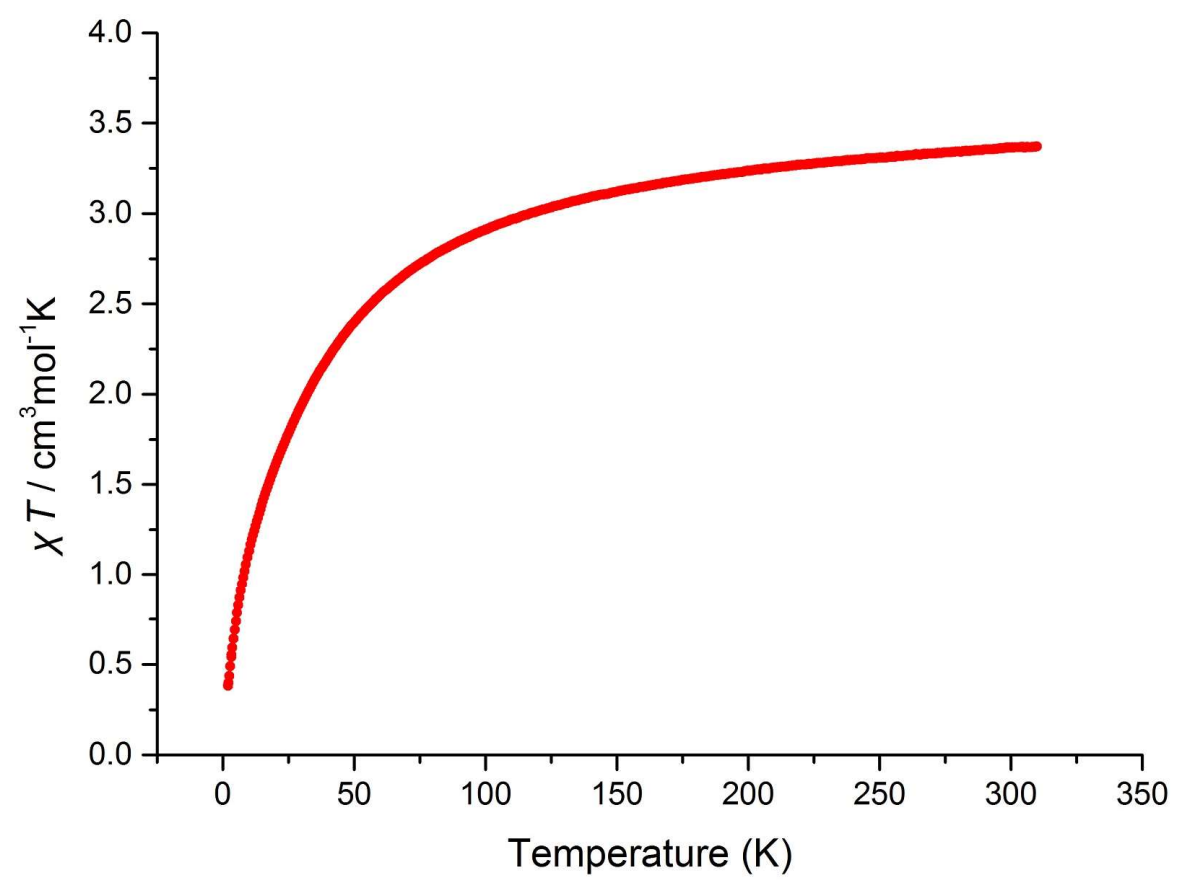

Figure S19. Variable-temperature magnetic data of complex $\mathbf{5}$ as $\mu_{\text {eff }}$ vs $\mathrm{T}(\mathrm{a}), \chi \mathrm{T}$ vs $\mathrm{T}$ (b). 


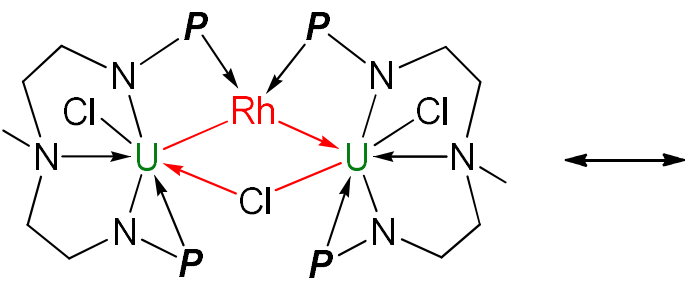

$4 \mathrm{~A}$

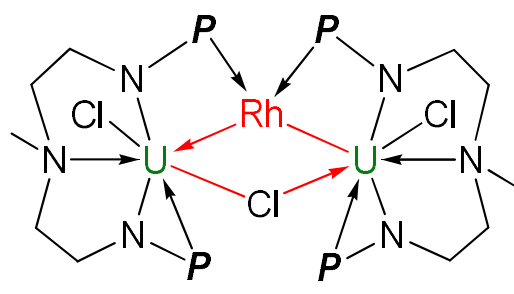

4B

Figure S20. Two major resonance structures of complex 4 . Both of $4 \mathrm{~A}$ and $\mathbf{4 B}$ contain a U-Rh $\sigma$ bond and a Rh-to- $\mathrm{U}$ dative bond. The formal oxidation state for $\mathrm{U}$ and $\mathrm{Rh}$ are $+\mathrm{IV}$ and $-\mathrm{I}$, respectively. 


\section{X-ray crystallographic analysis}

The crystallographic data were collected using a Bruker APEX-II CCD area detector with a radiation source of $\mathrm{Ga}(\mathrm{K} \alpha)(1.34139 \AA)$ or $\mathrm{Mo}(\mathrm{K} \alpha)(0.71073 \AA)$. Multiscan or empirical absorption corrections (SADABS) were applied. The structures were solved using Patterson methods, expanded using difference Fourier syntheses, and refined using full-matrix least squares fitting on $F^{2}$ using the Bruker SHELXTL-2014 program package. ${ }^{1,2}$ All non-hydrogen atoms were refined anisotropically. Hydrogen atoms were introduced at their geometric positions and refined as riding atoms. In complex $\mathbf{2}$, two alternative orientations for the heavy atoms were refined and resulted in site occupancies of $99 \%$ and $1 \%$ for U1 and U1', DELU was applied to the displacement ellipsoids of the atoms. Two alternative orientations for the ligand were refined and resulted in site occupancies of $90 \%$ and $10 \%$ for the $\mathrm{Cl}_{2}$ and $\mathrm{Cl}_{2}$ ' in complex 4 . In complex $\mathbf{5}$, fixed distance (DFIX) was used to rationalize the bond parameters of isopropyl group. There is an A-level alarm for structure $\mathbf{5}$ because a severely disordered solvent molecule cannot be built when evaluating the crystallographic information file (CIF) of complex 5 using the CheckCIF routine at http://checkcif.iucr.org/. CCDC1974258 (2), 1974259 (3), 1974260 (4), 1974261 (5) contain the crystallographic data for this paper. These data can be obtained free of charge from the Cambridge Crystallographic Data Centre (https://www.ccdc.cam.ac.uk/structures/). Details regarding the data collection and refinement for these complexes are given in Table S1.

Table S1. Crystal data and structural refinements for 2, 3, 4 and 5 .

\begin{tabular}{ccccc}
\hline & $\mathbf{2}$ & 3.2toluene & $\mathbf{4}$ & $\mathbf{5} \cdot 2 \mathrm{THF}$ \\
\hline empirical formula & $\mathrm{C}_{21} \mathrm{H}_{47} \mathrm{Cl}_{2} \mathrm{~N}_{3} \mathrm{OP}_{2}$ & $\mathrm{C}_{48} \mathrm{H}_{94} \mathrm{Cl}_{5} \mathrm{~N}_{6} \mathrm{P}_{4}$ & $\mathrm{C}_{34} \mathrm{H}_{78} \mathrm{Cl}_{3} \mathrm{~N}_{6} \mathrm{P}_{4}$ & $\mathrm{C}_{76} \mathrm{H}_{172} \mathrm{~N}_{14} \mathrm{P}_{8} \mathrm{O}_{2}$ \\
& $\mathrm{U}$ & $\mathrm{RhU}_{2}$ & $\mathrm{RhU}_{2}$ & $\mathrm{Rh}_{2} \mathrm{U}_{4}$ \\
formula weight & 728.48 & 1635.39 & 1380.22 & 2719.97 \\
temperature, $\mathrm{K}$ & $193(2)$ & $193(2)$ & $193(2)$ & $193(2)$ \\
wavelength, $\AA$ & 1.34139 & 0.71073 & 0.71073 & 1.34139 \\
crystal system & Triclinic & Triclinic & Monoclinic & Monoclinic \\
space group & $P-1$ & $P-1$ & $C 2 / c$ & $C 2 / c$ \\
$a, \AA$ & $8.2124(3)$ & $12.3662(5)$ & $25.734(2)$ & $32.1957(9)$ \\
$b, \AA$ & $11.5691(5)$ & $14.2438(7)$ & $10.2187(10)$ & $15.7190(3)$ \\
\hline
\end{tabular}




\begin{tabular}{|c|c|c|c|c|}
\hline$c, \AA$ & $16.9995(7)$ & $19.4960(7)$ & $22.353(3)$ & $20.6940(4)$ \\
\hline$\alpha,{ }^{\circ}$ & $97.5210(10)$ & $87.3920(10)$ & 90 & 90 \\
\hline$\beta,^{\circ}$ & 101.9180(10) & $78.4580(10)$ & $124.312(3)$ & $104.947(2)$ \\
\hline$\gamma,{ }^{\circ}$ & $104.5500(10)$ & $64.7550(10)$ & 90 & 90 \\
\hline$V, \AA^{3}$ & $1500.87(11)$ & $3040.2(2)$ & 4855.3(9) & $10118.6(4)$ \\
\hline$Z$ & 2 & 2 & 4 & 4 \\
\hline$d_{\text {calcd }}, \mathrm{g} \mathrm{cm}^{-3}$ & 1.612 & 1.786 & 1.888 & 1.785 \\
\hline$\mu, \mathrm{mm}^{-1}$ & 13.272 & 5.944 & 7.318 & 16.143 \\
\hline$F(000)$ & 716.0 & 1596.0 & 2656.0 & 5280.0 \\
\hline \multirow[t]{2}{*}{ crystal size, mm } & $0.10 \times 0.10 \times$ & $0.10 \times 0.10 \times$ & $0.10 \times 0.10 \times$ & $0.10 \times 0.10 \times$ \\
\hline & 0.10 & 0.10 & 0.10 & 0.10 \\
\hline$\theta_{\max },{ }^{\circ}$ & 52.990 & 24.996 & 25.010 & 53.875 \\
\hline \multirow[t]{2}{*}{ reflns collected } & 16823 & 22644 & 17338 & 56892 \\
\hline & $5128\left[\mathrm{R}_{\mathrm{int}}=\right.$ & $10593\left[\mathrm{R}_{\mathrm{int}}=\right.$ & $4289\left[\mathrm{R}_{\text {int }}=\right.$ & $9246\left[\mathrm{R}_{\mathrm{int}}=\right.$ \\
\hline \multirow[t]{2}{*}{ indep reflns } & $0.0609, \mathrm{R}_{\text {sigma }}=$ & $0.0418, \mathrm{R}_{\text {sigma }}=$ & $0.0729, \mathrm{R}_{\text {sigma }}=$ & $0.0838, \mathrm{R}_{\text {sigma }}=$ \\
\hline & $0.0536]$ & $0.0551]$ & $0.0649]$ & $0.0582]$ \\
\hline data/restraints/params & $5128 / 45 / 280$ & $10593 / 84 / 591$ & $4289 / 0 / 245$ & $9246 / 2 / 490$ \\
\hline goodness-of-fit on $F^{2}$ & 1.046 & 1.046 & 1.042 & 1.140 \\
\hline \multirow{2}{*}{ final $R(I>2 \sigma(I))$} & $\mathrm{R}_{1}=0.0429$ & $R_{1}=0.0379$ & $\mathrm{R}_{1}=0.0362$ & $\mathrm{R}_{1}=0.0397$ \\
\hline & $\mathrm{wR}_{2}=0.1156$ & $w R_{2}=0.0991$ & $\mathrm{wR}_{2}=0.0784$ & $\mathrm{wR}_{2}=0.0836$ \\
\hline \multirow{2}{*}{$R$ indices (all data) } & $\mathrm{R}_{1}=0.0437$ & $R_{1}=0.0447$ & $\mathrm{R}_{1}=0.0523$ & $\mathrm{R}_{1}=0.0672$ \\
\hline & $\mathrm{wR}_{2}=0.1163$ & $w R_{2}=0.1030$ & $\mathrm{wR}_{2}=0.0871$ & $\mathrm{wR}_{2}=0.0920$ \\
\hline \multicolumn{5}{|l|}{ Residual electron } \\
\hline $\begin{array}{c}\text { density }\left(\mathrm{e} . \AA^{-3}\right) \\
\max / \min \end{array}$ & $2.23 /-2.11$ & $2.54 /-1.71$ & $1.50 /-0.88$ & $1.77 /-1.13$ \\
\hline
\end{tabular}




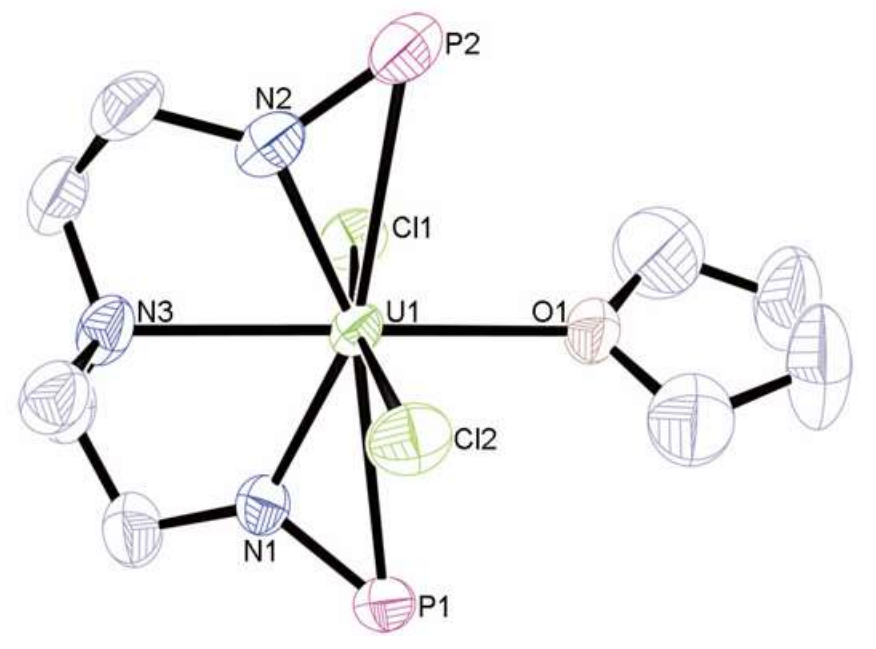

Figure S21. X-ray molecular structure of complex 2 drawn with 50\% probability. Hydrogen atoms and isopropyl moieties in $\mathrm{P}^{i} \mathrm{Pr}_{2}$ are omitted for clarity.

Table S2. Selected bond distances $(\AA)$ for $\mathbf{2}$.

$\begin{array}{lccc}\text { U1-C11 } & 2.6646(19) & \mathrm{U} 1-\mathrm{Cl} 2 & 2.669(2) \\ \mathrm{U} 1-\mathrm{P} 1 & \mathrm{U} 1-\mathrm{P} 2 & 3.049(2) \\ \mathrm{U} 1-\mathrm{O} 1 & 3.035(2) & \mathrm{U} 1-\mathrm{N} 1 & 2.221(6) \\ \mathrm{U} 1-\mathrm{N} 2 & 2.482(5) & \mathrm{U} 1-\mathrm{N} 3 & 2.587(7)\end{array}$

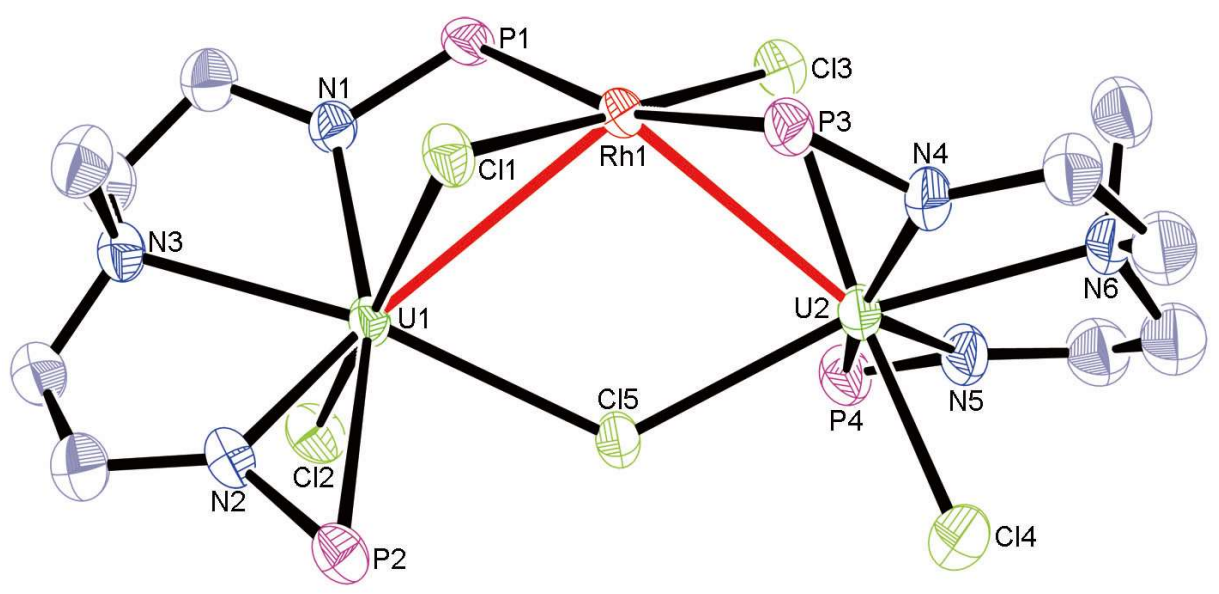

Figure S22. X-ray molecular structure of complex 3 drawn with 50\% probability. Hydrogen atoms and isopropyl moieties in $\mathrm{P}^{i} \mathrm{Pr}_{2}$ are omitted for clarity. 
Table S3. Selected bond distances $(\AA)$ for 3.

$\begin{array}{cccc}\text { U1-Rh1 } & 3.3177(5) & \mathrm{U} 1-\mathrm{Cl} 1 & 2.7543(14) \\ \mathrm{U} 1-\mathrm{Cl} 5 & 2.8359(15) & \mathrm{U} 1-\mathrm{Cl} 2 & 2.6343(15) \\ \mathrm{U} 1-\mathrm{P} 1 & \mathrm{U} 1-\mathrm{P} 2 & 3.0332(18) \\ \mathrm{U} 1-\mathrm{N} 3 & \mathrm{U} 1-\mathrm{N} 1 & 2.271(5) \\ \mathrm{U} 1-\mathrm{N} 2 & \mathrm{U} 2-\mathrm{Rh} 1 & 3.2609(5) \\ \mathrm{U} 2-\mathrm{Cl} 1 & \mathrm{U} 2-\mathrm{Cl} 3 & 2.7486(17) \\ \mathrm{U} 2-\mathrm{P} 3 & \mathrm{U} 2-\mathrm{Cl} 1 & 2.651(2) \\ \mathrm{U} 2-\mathrm{P} 4 & 2.7957(14) & \mathrm{U} 2-\mathrm{N} 6 & 2.617(5) \\ \mathrm{U} 2-\mathrm{N} 4 & 3.2014(16) & \mathrm{U} 2-\mathrm{N} 5 & 2.212(6) \\ \text { Rh1-Cl1 } & 2.262(5) & \mathrm{Rh} 1-\mathrm{Cl} 3 & 2.3616(17) \\ \text { Rh1-P1 } & 2.3644(16) & \mathrm{R} 1-\mathrm{P} 3 & 2.3324(16)\end{array}$

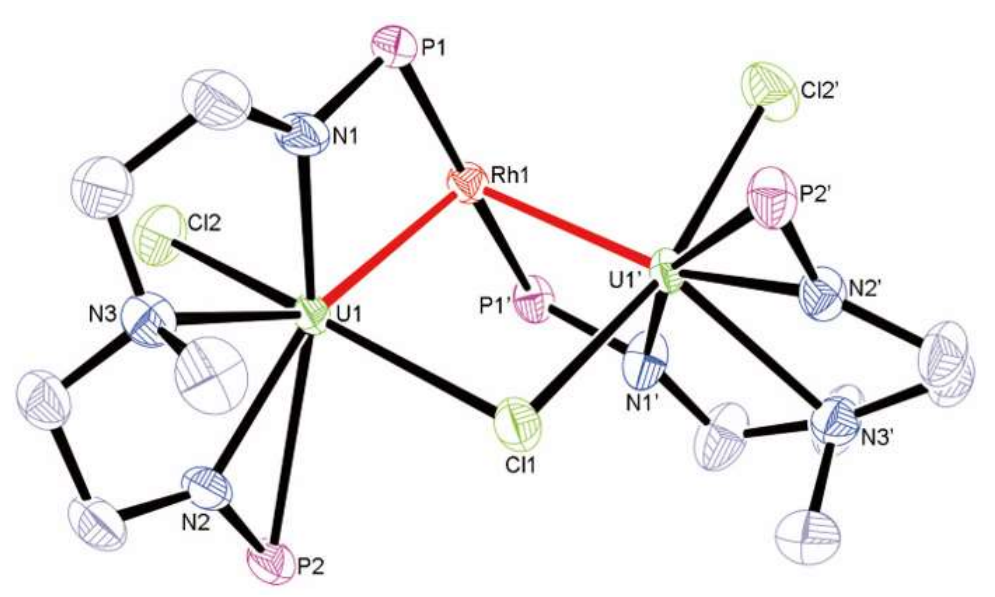

Figure S23. X-ray molecular structure of complex 4 drawn with 50\% probability. Hydrogen atoms and isopropyl moieties in $\operatorname{P}^{i} \operatorname{Pr}_{2}$ are omitted for clarity.

Table S4. Selected bond distances $(\AA)$ for 4.

$\begin{array}{llcr}\text { U1-N1 } & 2.310(6) & \mathrm{U} 1-\mathrm{N} 2 & 2.255(6) \\ \mathrm{U} 1-\mathrm{N} 3 & 2.642(6) & \mathrm{U} 1-\mathrm{Rh} 1 & 2.6555(6) \\ \mathrm{U} 1-\mathrm{Cl1} & 2.8055(18) & \mathrm{U} 1-\mathrm{P} 1 & 3.1256(19) \\ \mathrm{U1} 1-\mathrm{P} 2 & 3.000(2) & \mathrm{U} 1-\mathrm{U} 1 & 4.0397(6) \\ \mathrm{Rh1-P1} & 2.370(2) & & \end{array}$




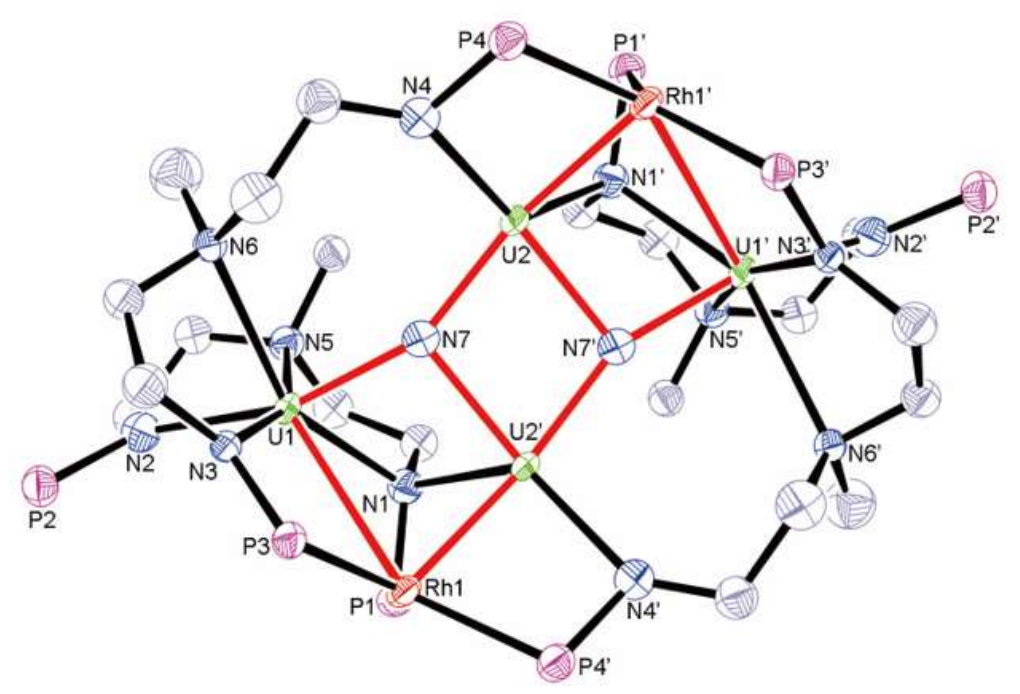

Figure S24. X-ray molecular structure of complex 5 drawn with 50\% probability. Hydrogen atoms and isopropyl moieties in $\mathrm{P}^{i} \mathrm{Pr}_{2}$ are omitted for clarity.

Table S5. Selected bond distances $(\AA)$ for $\mathbf{5}$.

\begin{tabular}{|c|c|c|c|}
\hline U2-N7 & $2.154(7)$ & U1-N7 & $2.158(7)$ \\
\hline U2-N7' & $2.302(6)$ & U1-N3 & $2.282(7)$ \\
\hline $\mathrm{U} 2-\mathrm{N} 4$ & $2.371(7)$ & U1-N2 & $2.461(7)$ \\
\hline U2-Rh1' & $2.5139(7)$ & U1-N1 & $2.552(6)$ \\
\hline U2-N1' & $2.605(7)$ & U1-N5 & $2.732(7)$ \\
\hline U2-P4 & $3.120(2)$ & U1-N6 & $2.847(7)$ \\
\hline U2-P1' & $3.135(2)$ & U1-P3 & $3.203(2)$ \\
\hline U2-U1' & $3.4677(4)$ & U1-Rh1 & $3.2160(7)$ \\
\hline U2-U2' & $3.4730(6)$ & U1-P1 & $3.384(2)$ \\
\hline Rh1-P3 & $2.324(2)$ & Rh1-P1 & $2.333(2)$ \\
\hline Rh1-P4' & $2.438(2)$ & U1-U2' & $3.4677(4)$ \\
\hline
\end{tabular}




\section{Theoretical Calculations}

The Gaussian09 program suite was used for all quantum-chemical calculations. ${ }^{3}$ We used Becke's 3-parameter hybrid version, ${ }^{4}$ combined with the non-local correlation functional provided by Perdew and Wang, ${ }^{5}$ denoted as B3PW91. The relativistic energy-consistent small-core pseudopotential of the Stuttgart-Köln ECP library was used in combination with its adapted segmented basis set to represent a uranium atom. ${ }^{6-8}$ In specific cases (associated with hexametallic complexes), the $f$-in-core RECPs adapted to a U(IV) core configuration $\left(5 f^{2}\right)$ was used in association with the adapted basis set. For phosphorus, chlorine and rhodium, the quasi-relativistic energyadjusted ab-initio pseudopotentials were used, along with their corresponding energy-optimized valence basis sets. ${ }^{9-10}$ For all the atoms, the $6-31 \mathrm{G}(\mathrm{d})$ basis set was used. ${ }^{11-12}$ In all computations no constraints were imposed on the geometry. A full geometry optimization was performed for each structure using Schlegel's analytical gradient method ${ }^{13}$ and the attainment of the energy minimum was verified by calculating the vibrational frequencies that result in the absence of imaginary eigenvalues. All stationary points have been identified for the minimum (number of imaginary frequencies, Nimag $=0$ ). The NBO analysis ${ }^{14}$ was carried out on the optimized structures using the module included in the Gaussian package. The Chemcraft graphical program was used for the 3D representations of the structures and the orbital plots. ${ }^{15}$

\begin{tabular}{|l|l|l|l|l|l|l|}
\hline Large Core & Small Core & Dispersion & $\mid \Delta(\mathrm{LC}-$ & $\mid \Delta(\mathrm{LC}-$ & $\mid \Delta(\mathrm{SC}-$ \\
$(\mathrm{SC})$ & $(\mathrm{D})$ & $\mathrm{SC}) \mid \%$ & $\mathrm{D}) \mid \%$ & $\mathrm{D}) \mid \%$ \\
\hline Rh-Cl1 & 2.45613 & 2.46059 & 2.43463 & 0.18 & 0.88 & 1.07 \\
\hline Rh-Cl2 & 2.45516 & 2.45767 & 2.43415 & 0.10 & 0.86 & 0.97 \\
\hline
\end{tabular}




\begin{tabular}{|c|c|c|c|c|c|c|}
\hline $\mathrm{Rh}-\mathrm{P} 1$ & 2.38402 & 2.36994 & 2.33646 & 0.59 & 2.04 & 1.43 \\
\hline $\mathrm{Rh}-\mathrm{P} 2$ & 2.38560 & 2.37088 & 2.34101 & 0.62 & 1.90 & 1.28 \\
\hline U1-Cl1 & 2.87296 & 2.80392 & 2.78396 & 2.46 & 3.20 & 0.72 \\
\hline U1-Cl2 & 2.78396 & 2.83436 & 2.80678 & 1.78 & 0.81 & 0.98 \\
\hline $\mathrm{U} 1-\mathrm{Cl} 3$ & 2.71962 & 2.66478 & 2.65861 & 2.06 & 2.29 & 0.23 \\
\hline U1-N1 & 2.27680 & 2.21599 & 2.19575 & 2.74 & 3.69 & 0.92 \\
\hline U1-N2 & 2.64864 & 2.63900 & 2.61701 & 0.37 & 1.21 & 0.84 \\
\hline U1-N3 & 2.31595 & 2.25979 & 2.24553 & 2.49 & 3.14 & 0.64 \\
\hline U2-Cl1 & 2.87735 & 2.81198 & 2.79095 & 2.32 & 3.10 & 0.75 \\
\hline $\mathrm{U} 2-\mathrm{Cl} 2$ & 2.87694 & 2.83904 & 2.81259 & 1.33 & 2.29 & 0.94 \\
\hline $\mathrm{U} 2-\mathrm{Cl} 3$ & 2.72277 & 2.66640 & 2.65775 & 2.11 & 2.45 & 0.33 \\
\hline U2-N1 & 2.31250 & 2.25734 & 2.24582 & 2.44 & 2.97 & 0.51 \\
\hline U2-N2 & 2.65130 & 2.64351 & 2.61682 & 0.29 & 1.32 & 1.02 \\
\hline U2-N3 & 2.27767 & 2.21427 & 2.19692 & 2.86 & 3.68 & 0.79 \\
\hline U1-Rh & 3.44188 & 3.43071 & 3.30683 & 0.33 & 4.08 & 3.75 \\
\hline U2-Rh & 3.39761 & 3.40069 & 3.27917 & 0.09 & 3.61 & 3.71 \\
\hline
\end{tabular}

\begin{tabular}{|l|l|l|l|}
\hline Charges & LC & SC & D \\
\hline Rh & -0.774042 & -0.52607 & -0.56959 \\
\hline
\end{tabular}




\begin{tabular}{|c|c|c|c|c|c|c|}
\hline Complex 4 & & & & & & \\
\hline Distances & $\begin{array}{ll}\text { Large } & \text { Core } \\
\text { (LC) } & \end{array}$ & $\begin{array}{l}\text { Small Core } \\
(\mathrm{SC})\end{array}$ & $\begin{array}{l}\text { Dispersion } \\
\text { (D) }\end{array}$ & $\begin{array}{l}\mid \Delta(\mathrm{LC}- \\
\mathrm{SC}) \mid \%\end{array}$ & $\begin{array}{l}\mid \Delta(\mathrm{LC}- \\
\mathrm{D}) \mid \%\end{array}$ & $\begin{array}{l}\mid \Delta(\mathrm{SC}- \\
\mathrm{D}) \mid \%\end{array}$ \\
\hline Rh-P1 & 2.34995 & 2.35670 & 2.33121 & 0.29 & 0.80 & 1.09 \\
\hline $\mathrm{Rh}-\mathrm{P} 2$ & 2.34769 & 2.35936 & 2.33113 & 0.49 & 0.71 & 1.21 \\
\hline U1-Cl1 & 2.73386 & 2.66701 & 2.66101 & 2.51 & 2.74 & 0.23 \\
\hline $\mathrm{U} 1-\mathrm{Cl} 2$ & 2.90908 & 2.85989 & 2.83303 & 1.72 & 2.68 & 0.95 \\
\hline U1-N1 & 2.31509 & 2.25791 & 2.23207 & 2.53 & 3.72 & 1.16 \\
\hline U1-N2 & 2.69763 & 2.71051 & 2.67856 & 0.48 & 0.71 & 1.19 \\
\hline U1-N3 & 2.32099 & 2.26994 & 2.25799 & 2.25 & 2.79 & 0.53 \\
\hline U2-Cl1 & 2.73390 & 2.66676 & 2.66047 & 2.52 & 2.76 & 0.24 \\
\hline $\mathrm{U} 2-\mathrm{Cl} 2$ & 2.90784 & 2.86314 & 2.83342 & 1.56 & 2.63 & 1.05 \\
\hline U2-N1 & 2.32401 & 2.27455 & 2.26109 & 2.17 & 2.78 & 0.60 \\
\hline U2-N2 & 2.69000 & 2.70657 & 2.67012 & 0.61 & 0.74 & 1.37 \\
\hline U2-N3 & 2.31603 & 2.25973 & 2.23539 & 2.49 & 3.61 & 1.09 \\
\hline U1-Rh & 2.87729 & 2.77033 & 2.72368 & 3.86 & 5.64 & 1.71 \\
\hline U2-Rh & 2.87009 & 2.75826 & 2.71764 & 4.05 & 5.61 & 1.49 \\
\hline
\end{tabular}




\begin{tabular}{|l|l|l|l|}
\hline Charges & LC & SC & D \\
\hline Rh & -1.002815 & -0.77027 & -0.78081 \\
\hline
\end{tabular}


Complex 5

\begin{tabular}{|l|l|l|l|}
\hline Distances & Exp. & LC & $\mid$ (LC-Exp) $\%$ \\
\hline U1---U2 & 3.46 & 3.53 & 1.98 \\
\hline U1-Rh1 & 3.21 & 3.23 & 0.62 \\
\hline U2-Rh1' & 2.51 & 2.80 & 10.36 \\
\hline U1-N3 & 2.28 & 2.34 & 2.56 \\
\hline U1-N5 & 2.73 & 2.84 & 3.87 \\
\hline U1-N2 & 2.46 & 2.54 & 3.15 \\
\hline U1-N1 & 2.55 & 2.65 & 3.77 \\
\hline U1-N6 & 2.85 & 2.88 & 1.04 \\
\hline U1-N7 & 2.17 & 2.24 & 3.57 \\
\hline U1-P3 & 3.20 & 3.30 & 3.03 \\
\hline U1-P1 & 3.38 & 3.46 & 2.31 \\
\hline U2---U2' & 3.47 & 3.53 & 1.70 \\
\hline U2-N4 & 2.37 & 2.42 & 2.07 \\
\hline U2-N1' & 2.60 & 2.60 & - \\
\hline & 2.30 & 2.30 & - \\
\hline & 2.44 & 2.45 & 0.41 \\
\hline & & & \\
\hline & & & \\
\hline & & & \\
\hline & & & \\
\hline & & & \\
\hline
\end{tabular}




\begin{tabular}{|l|l|l|l|}
\hline Rh1-P3 & 2.32 & 2.34 & 0.85 \\
\hline U2'-N7' & 2.15 & 2.25 & 4.44 \\
\hline U2-P1' & 3.11 & 3.25 & 4.31 \\
\hline U2-P4 & 3.12 & 2.81 & 11.03 \\
\hline
\end{tabular}

\begin{tabular}{|l|l|}
\hline Charges & LC \\
\hline Rh1 & -1.23283 \\
\hline
\end{tabular}

$\underline{\text { Complex } 3}$

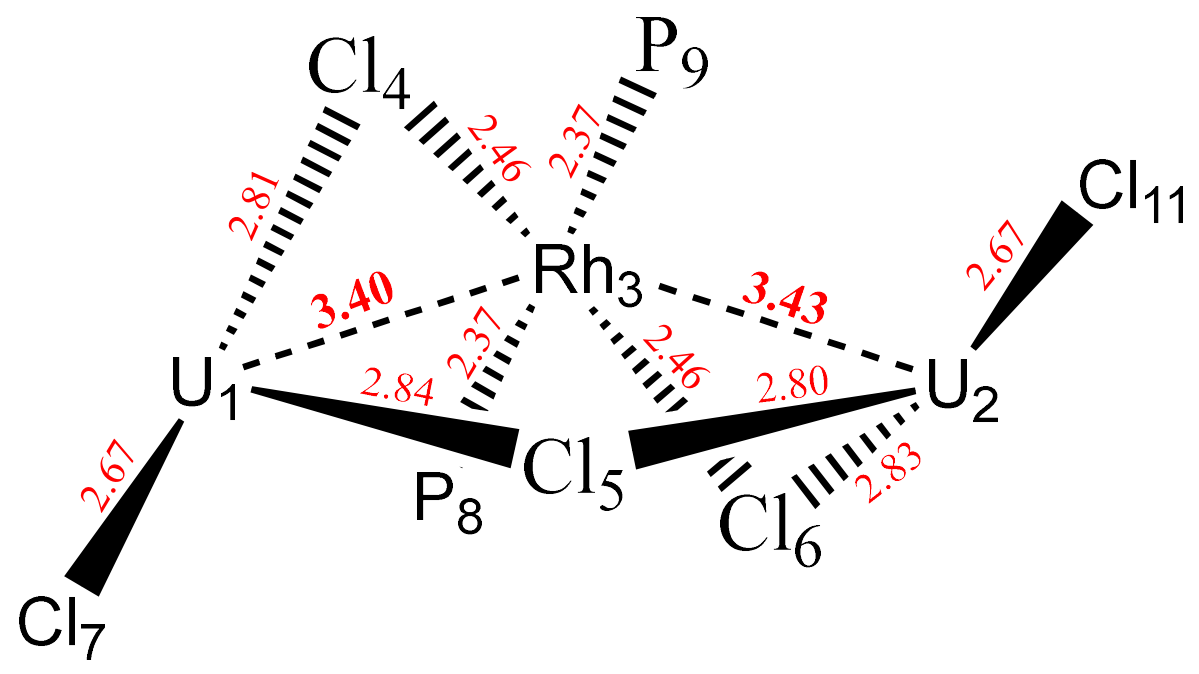

Natural charge

\begin{tabular}{|c|c|c|c|c|}
\hline $\mathbf{U}_{\mathbf{1}}=$ & $\mathbf{U}_{\mathbf{2}}=$ & $\mathbf{R h}_{\mathbf{3}}=-0.52607$ & $\mathbf{C l}_{\mathbf{4}}=-$ & $\begin{array}{c}\mathbf{C l}_{\mathbf{5}}=- \\
0.58340\end{array}$ \\
0.58227 & & 0.15652 & 0.10696 \\
\hline $\mathbf{C l}_{\mathbf{6}}=-0.15435$ & $\mathbf{C l}_{\mathbf{7}}=-0.29128$ & $\mathbf{P}_{\mathbf{8}}=1.49914$ & $\mathbf{P}_{\mathbf{9}}=1.49510$ & $\mathbf{C l}_{\mathbf{1 1}}=-0.28979$ \\
\hline
\end{tabular}




\section{Natural Electron Configuration}

$\mathbf{U}_{1} \quad[$ core]7S( 0.21)5f( 2.93)6d( 1.59)7p( 0.42)

$\mathbf{U}_{2} \quad[$ core $7 \mathrm{~S}(0.21) 5 \mathrm{f}(2.93) 6 \mathrm{~d}(1.59) 7 \mathrm{p}(0.42)$

$\mathbf{R h}_{3} \quad$ [core] $5 \mathrm{~S}(0.32) 4 \mathrm{~d}(8.74) 5 \mathrm{p}(0.46) 5 \mathrm{~d}(0.01) 6 \mathrm{~d}(0.01)$

\section{$\underline{\text { Wiberg bond index }}$}

$$
\begin{array}{cccc}
\mathbf{U}_{\mathbf{1}}-\mathbf{R h}=0.2819 & \mathbf{U}_{\mathbf{1}}-\mathbf{C l}_{\mathbf{4}}=0.7843 \quad \mathbf{U}_{\mathbf{1}}-\mathbf{C l}_{\mathbf{7}}=1.1565 \\
\mathbf{U}_{\mathbf{2}}-\mathbf{R h}=0.2677 & \mathbf{U}_{\mathbf{2}}-\mathbf{C l}_{\mathbf{6}}=0.7912 & \mathbf{U}_{\mathbf{2}}-\mathbf{C l}_{\mathbf{1 1}}=1.1609 \\
\mathbf{R h}-\mathbf{C l}_{\mathbf{4}}=0.4497 & \mathbf{R h}-\mathbf{C l}_{\mathbf{6}}=0.4467 & \mathbf{R h}-\mathbf{P h}_{\mathbf{8}}=0.5440 \quad \mathbf{R h}-\mathbf{P h} \mathbf{9}=0.5447
\end{array}
$$

\begin{tabular}{|c|c|}
\hline Alpha & Beta \\
\hline $\begin{array}{c}\text { (0.97363) BD ( 1) U } \quad \mathbf{1} \text {-Cl } 4 \\
(13.68 \%) \mathrm{U} 1 \mathrm{~s}(10.93 \%) \mathrm{p}(20.40 \%) \mathrm{d}(39.50 \%) \mathrm{f}(29.17 \%) \\
(86.32 \%) \mathrm{Cl} 4 \mathrm{~s}(45.87 \%) \mathrm{p}(54.13 \%)\end{array}$ & 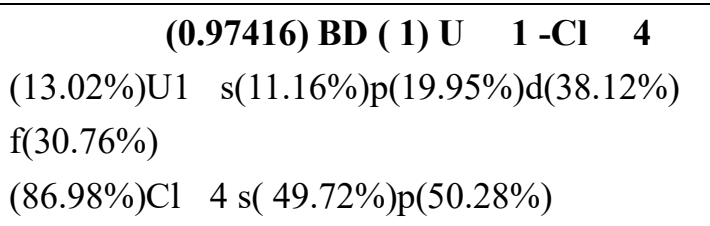 \\
\hline $\begin{array}{l}\text { (0.97081) BD ( 1) U } 1 \text {-Cl } 5 \\
(13.78 \%) \mathrm{U} 1 \\
\mathrm{~s}(11.39 \%) \mathrm{p}(16.72 \%) \mathrm{d}(41.35 \%) \mathrm{f}(30.53 \%) \\
(86.22 \%) \mathrm{Cl} 5 \mathrm{~s}(37.04 \%) \mathrm{p}(62.96 \%)\end{array}$ & $\begin{array}{l}\text { (0.97041) BD ( 1) U 1 -Cl } 5 \\
(13.29 \%) \mathrm{U} 1 \\
\mathrm{~s}(11.44 \%) \mathrm{p}(17.14 \%) \mathrm{d}(40.61 \%) \mathrm{f}(30.80 \%) \\
(86.71 \%) \mathrm{Cl} 5 \mathrm{~s}(37.86 \%) \mathrm{p}(62.14 \%)\end{array}$ \\
\hline 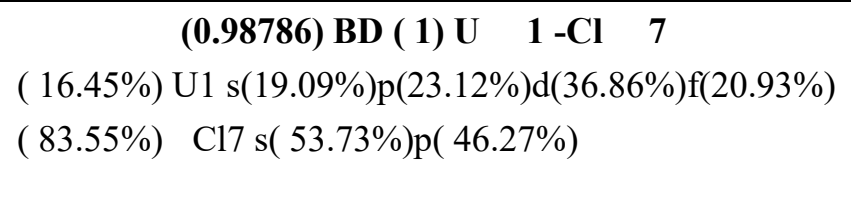 & $\begin{array}{l}\text { (0.98977) BD ( 1) U } \quad \text { 1 -Cl } 7 \\
(15.83 \%) \mathrm{U} 1 \quad \text { s(18.88\%)p }(22.92 \%) \mathrm{d}(35.74 \%) \mathrm{f} \\
(22.45 \%) \\
(84.17 \%) \mathrm{Cl} 7 \mathrm{~s}(57.84 \%) \mathrm{p}(42.16 \%)\end{array}$ \\
\hline
\end{tabular}

\begin{tabular}{|c|c|}
\hline Alpha & Beta \\
\hline $\begin{array}{l}\text { (0.97128) BD ( 1) U } \quad 2 \text {-Cl } \quad \mathbf{5} \\
(13.90 \%) \mathrm{U} 2 \mathrm{~s}(11.54 \%) \mathrm{p}(16.37 \%) \mathrm{d}(41.15 \%) \mathrm{f}(30.94 \%) \\
(86.10 \%) \mathrm{Cl} \quad 5 \mathrm{~s}(37.10 \%) \mathrm{p}(62.90 \%)\end{array}$ & $\begin{array}{l}\text { (0.97096) BD ( 1) U } 2 \text {-Cl } 5 \\
(13.39 \%) \mathrm{U} 2 \\
\mathrm{~s}(11.59 \%) \mathrm{p}(16.78 \%) \mathrm{d}(40.57 \%) \mathrm{f}(31.05 \%) \\
(86.61 \%) \mathrm{Cl} 5 \mathrm{~s}(38.04 \%) \mathrm{p}(61.96 \%)\end{array}$ \\
\hline $\begin{array}{l}\text { (0.98083) BD ( 1) U } 2 \text {-Cl } 6 \\
(13.09 \%) \mathrm{U} 2 \\
\mathrm{~s}(11.14 \%) \mathrm{p}(20.33 \%) \mathrm{d}(39.15 \%) \mathrm{f}(29.38 \%) \\
(86.91 \%) \mathrm{Cl} 6 \mathrm{~s}(35.90 \%) \mathrm{p}(64.10 \%)\end{array}$ & 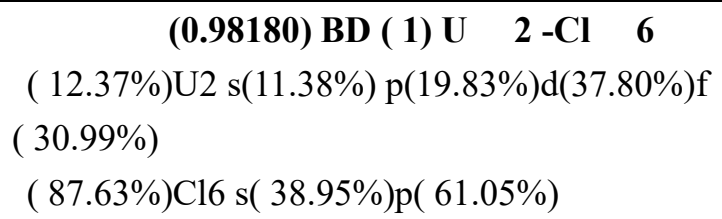 \\
\hline$(0.98806)$ BD ( 1) U & $(0.98997)$ BD ( 1) U \\
\hline
\end{tabular}

\section{Bond}


$(16.48 \%) \mathrm{U} 2$

s( 19.02\%)p( 23.21\%)d( 36.70\%)f( 21.06\%)

$(83.52 \%) C 111$ s( $53.76 \%) p(46.24 \%)$
( 15.85\%)U 2 s( 18.79\%)p( 22.99\%)d( 35.62\%)f $(22.60 \%)$

( 84.15\%)C111 s( 57.89\%)p( 42.11\%)

\begin{tabular}{|c|c|}
\hline Alpha & Beta \\
\hline $\begin{array}{l}\text { (0.93215) BD ( 1)Rh } \quad \text { 3 -Cl } \quad \mathbf{4} \\
(16.62 \%) R h 3 \mathrm{~s}(29.82 \%) \mathrm{p}(11.33 \%) \mathrm{d}(58.85 \%) \\
(83.38 \%) \mathrm{Cl} 4 \mathrm{~s}(12.47 \%) \quad \mathrm{p}(87.53 \%)\end{array}$ & $\begin{array}{l}\text { (0.93297) BD ( 1)Rh } \quad 3 \text {-Cl } \\
\begin{array}{l}\text { ( } 16.43 \%) \\
\mathrm{sh} 3\end{array} \\
\mathrm{~s}(30.06 \%) \mathrm{p}(10.86 \%) \mathrm{d}(59.08 \%) \\
(83.57 \%) \quad \mathrm{Cl} 4 \mathrm{~s}(11.56 \%) \mathrm{p}(88.44 \%)\end{array}$ \\
\hline $\begin{array}{r}\text { (0.88057) BD ( 1)Rh } \mathbf{3}-\mathbf{P} \quad \mathbf{8} \\
(26.84 \%) \mathrm{Rh} 3 \mathrm{~s}(43.30 \%) \mathrm{p}(16.58 \%) \mathrm{d}(40.11 \%) \\
(73.16 \%) \mathrm{P} 8 \mathrm{~s}(36.05 \%) \mathrm{p}(63.82 \%) \mathrm{d}(0.13 \%)\end{array}$ & $\begin{array}{c}\text { (0.88202) BD ( 1)Rh } \quad 3 \text { - P } \quad \mathbf{8} \\
(26.72 \%) \operatorname{Rh} 3 \mathrm{~s}(43.79 \%) \mathrm{p}(16.35 \%) \mathrm{d}(39.86 \%) \\
(73.28 \%) \mathrm{P} 8 \quad \mathrm{~s}(36.29 \%) \mathrm{p}(63.58 \%) \mathrm{d}(\quad 0.13 \%)\end{array}$ \\
\hline
\end{tabular}

Alpha donation:

\begin{tabular}{|c|c|c|}
\hline Donor & Acceptor & Energy $\left(\right.$ kcal.mol $\left.^{-1}\right)$ \\
\hline$U 1$ & \multirow{2}{*}{$R h 3$} & 17.03 \\
\hline$U 2$ & & 34.29 \\
\hline$R h 3$ & $U 1$ & $45.71(47 \% C R-53 \% L P)$ \\
\hline$R h 3$ & $U 2$ & $33.21(57 \% C R-43 \% L P)$ \\
\hline
\end{tabular}


Orbital

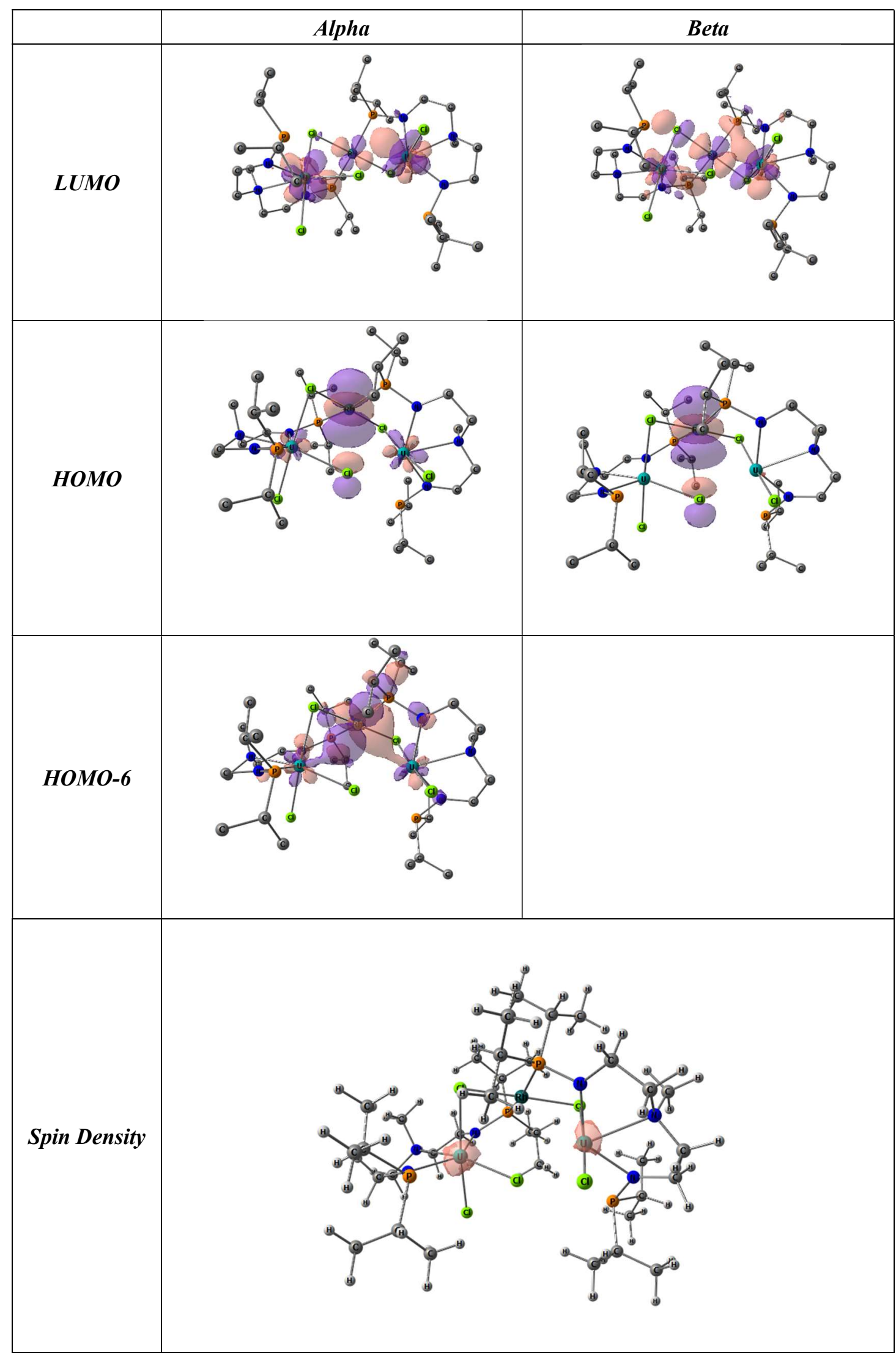




\begin{tabular}{|l|l|l|l|l|l|}
\hline & Exp. & Cal. & & Exp. & Cal. \\
\hline U1-Rh1 & $3.3177(5)$ & 3.40 & U2-Rh1 & $3.2609(5)$ & 3.40 \\
\hline U1-C11 & $2.7543(4)$ & 2.81 & U2-Cl3 & $2.7486(17)$ & 2.80 \\
\hline U1-C12 & $2.6343(15)$ & 2.67 & U2-C14 & $2.651(2)$ & 2.67 \\
\hline U1-C15 & $2.8359(15)$ & 2.84 & U2-C15 & $2.7957(14)$ & 2.83 \\
\hline U1-P1 & $3.2275(17)$ & & U2-P3 & $3.2014(16)$ & \\
\hline U1-P2 & $3.0332(18)$ & & U2-P4 & $3.0338(19)$ & \\
\hline U1-N1 & $2.271(5)$ & & U2-N4 & $2.262(5)$ & \\
\hline U1-N2 & $2.221(5)$ & & U2-N5 & $2.212(6)$ & \\
\hline U1-N3 & $2.595(5)$ & & U2-N6 & $2.617(5)$ & \\
\hline Rh1-C11 & $2.3644(16)$ & 2.46 & Rh1-P1 & $2.3385(17)$ & 2.37 \\
\hline Rh1-C13 & $2.3616(17)$ & 2.46 & Rh1-P3 & $2.3324(16)$ & 2.37 \\
\hline
\end{tabular}

\section{Complex 3 with dispersion}

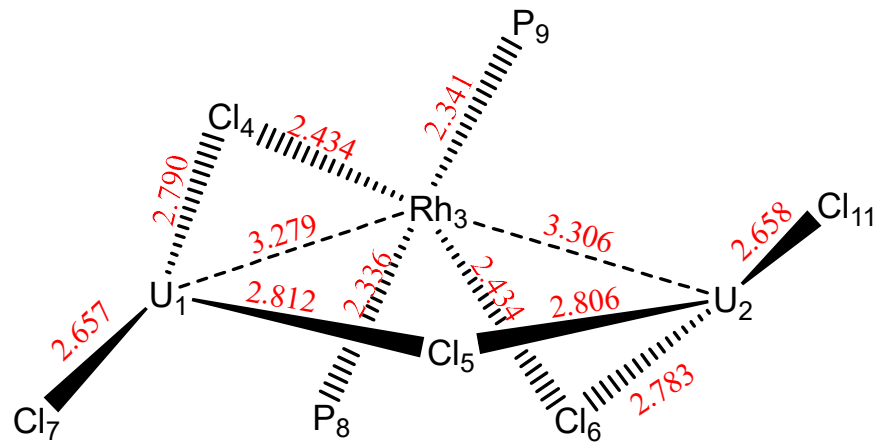

Natural Charge

\begin{tabular}{|c|c|c|c|c|}
\hline $\begin{array}{c}\mathbf{U}_{1}= \\
0.40804\end{array}$ & $\begin{array}{c}\mathbf{U}_{2}= \\
0.40841\end{array}$ & $\begin{array}{c}\mathbf{R h}_{3}=- \\
0.56959\end{array}$ & $\begin{array}{l}\mathbf{C l}_{4}=- \\
0.12157\end{array}$ & $\begin{array}{l}\mathbf{C l}_{5}=- \\
0.06851\end{array}$ \\
\hline $\begin{array}{l}\mathbf{C l}_{6}=- \\
0.11936\end{array}$ & $\mathbf{C l}_{7}=-0.27266$ & $\mathbf{P}_{8}=1.55409$ & $\begin{array}{l}\mathbf{P}_{9}= \\
1.54887\end{array}$ & $\begin{array}{c}\text { Cl11 }_{11}=- \\
0.27310\end{array}$ \\
\hline
\end{tabular}

\section{$\underline{\text { Natural Electron Configuration }}$}

U1 [core]7S( 0.21)5f( 2.97)6d( 1.66)7p( 0.44)

U2 [core]7S( 0.21)5f( 2.98)6d( 1.66)7p( 0.44)

Rh3 [core]5S( 0.32)4d( 8.77)5p( 0.49)5d( 0.01)6d( 0.01)

Wiberg bond index

$$
\begin{array}{ccc}
\mathbf{U}_{\mathbf{1}}-\mathbf{R h}= & 0.3298 \quad \mathbf{U}_{\mathbf{1}}-\mathbf{C l}_{\mathbf{4}}=0.8049 \quad \mathbf{U}_{\mathbf{1}}-\mathbf{C l}_{\mathbf{7}}=1.1742 \\
\mathbf{U}_{\mathbf{2}}-\mathbf{R h}= & 0.3148 \quad \mathbf{U}_{\mathbf{2}}-\mathbf{C l}_{\mathbf{6}}=0.8103 \quad \mathbf{U}_{\mathbf{2}}-\mathbf{C l}_{\mathbf{1 1}}=1.1741 \\
\mathbf{R h}-\mathbf{C l}_{\mathbf{4}}=0.4511 \quad \mathbf{R h}-\mathbf{C l}_{\mathbf{6}}=0.4495 \mathbf{R h}-\mathbf{P h}_{\mathbf{8}}=0.5274 \mathbf{R h}-\mathbf{P h} \mathbf{9}=0.5240
\end{array}
$$


Total Wiberg Index

\begin{tabular}{|l|l|l|}
\hline $\mathbf{U}_{\mathbf{1}}=6.3192$ & $\mathbf{U}_{\mathbf{2}}=6.3160$ & $\mathbf{R h}_{\mathbf{3}}=3.0445$ \\
\hline
\end{tabular}

\title{
Bond
}

\author{
1.BD ( 1) U 1 -Cl 4 \\ (13.98\%) $\quad 0.3738 * \mathrm{U} \quad 1 \mathrm{~s}(10.04 \%)$ p $1.98(19.90 \%)$ d $4.14(41.56 \%)$ f $2.84(28.50 \%)$ g $0.00(0.01 \%)$ \\ ( $86.02 \%) \quad 0.9275 * \mathrm{Cl} \quad 4 \mathrm{~s}(48.02 \%)$ p $1.08(51.98 \%)$ \\ 2.BD ( 1) U 1 -Cl 5 \\ ( $14.22 \%) \quad 0.3771^{*} \mathrm{U} \quad 1 \mathrm{~s}(10.66 \%)$ p $1.64(17.47 \%)$ d $3.98(42.37 \%)$ f $2.77(29.50 \%)$ g $0.00(\quad 0.01 \%)$ \\ $(85.78 \%) \quad 0.9262 * \mathrm{Cl} \quad 5 \mathrm{~s}(36.59 \%) \mathrm{p} 1.73(63.41 \%)$ \\ 3.BD ( 1) U 1 -Cl 7 \\ ( $16.82 \%) \quad 0.4101 * \mathrm{U} \quad 1 \mathrm{~s}(19.12 \%)$ p $1.19(22.74 \%) \mathrm{d} 2.00(38.27 \%)$ f $1.04(19.87 \%)$ g $0.00(\quad 0.01 \%)$ \\ $(83.18 \%) \quad 0.9121 * \mathrm{Cl} \quad 7$ s( $56.25 \%)$ p $0.78(43.75 \%)$ \\ 4.BD ( 1) U 1 - P 10 \\ $\begin{array}{llll}(17.80 \%) & 0.4219^{*} \mathrm{U} & 1 \mathrm{~s}(\mathbf{9 . 8 6} \%) \mathrm{p} 2.48(24.46 \%) \mathrm{d} .84(37.84 \%) \text { f } 2.82(27.84 \%) \mathrm{g} 0.00(0.00 \%)\end{array}$ \\ ( 82.20\%) $\quad 0.9067 * \mathrm{P} \quad 10 \mathrm{~s}(41.46 \%)$ p $1.41(58.47 \%) d 0.00(\quad 0.07 \%)$ \\ 5.BD ( 1) U 1 - N 13 \\ ( $9.23 \%) \quad 0.3038 * \mathrm{U} \quad 1 \mathrm{~s}(13.40 \%)$ p $1.09(14.65 \%) \mathrm{d} 2.50(33.54 \%)$ f $2.87(38.41 \%) \mathrm{g} 0.00(\quad 0.00 \%)$ \\ ( 90.77\%) $\quad 0.9527 * \mathrm{~N} \quad 13 \mathrm{~s}(25.32 \%)$ p 2.95( $74.67 \%)$ d $0.00(\quad 0.01 \%)$ \\ 6.BD ( 1) U 1 - N 14

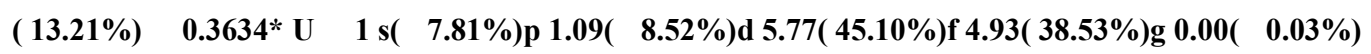 \\ ( 86.79\%) $\quad 0.9316^{*} \mathrm{~N} \quad 14 \mathrm{~s}(47.40 \%)$ p 1.11( $\left.52.59 \%\right)$ d $0.00(\quad 0.01 \%)$ \\ 7. BD ( 2) U 1 - N 14 \\ $\begin{array}{llll}(11.89 \%) & 0.3449 * \mathrm{U} & 1 \mathrm{~s}( & \mathbf{0 . 4 5} \%) \mathrm{p} 17.89(\quad 8.10 \%) \mathrm{d} 95.21(43.09 \%) f 99.99(48.35 \%) \mathrm{g} 0.03(\quad 0.01 \%)\end{array}$

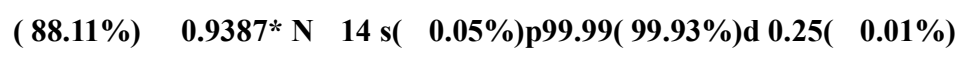 \\ 8.BD ( 1) U 1 - N 15 \\ ( $14.21 \%) \quad 0.3770^{*} \mathrm{U} \quad 1 \mathrm{~s}(12.15 \%)$ p $0.44(5.38 \%)$ d $3.82(46.39 \%)$ f $2.97(36.04 \%)$ g $0.00(\quad 0.04 \%)$ \\ ( 85.79\%) $\quad 0.9262 * \mathrm{~N} \quad 15 \mathrm{~s}(46.75 \%) \mathrm{p} 1.14(53.24 \%) \mathrm{d} 0.00(\quad 0.01 \%)$ \\ 9.BD ( 2) U 1 - N 15

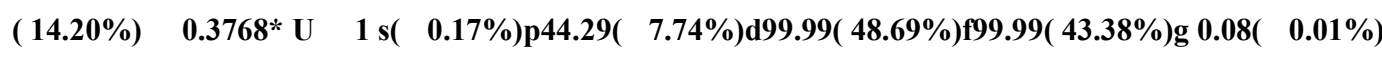

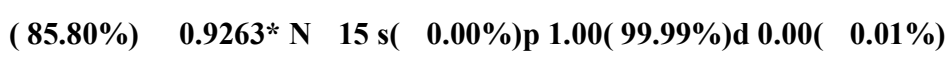

\section{BD ( 1) U 2 -Cl 5} \\ (14.34\%) $\quad 0.3787^{*} \mathrm{U} \quad 2$ s( $\left.10.62 \%\right)$ p $1.62(17.19 \%)$ d 3.99( $\left.42.32 \%\right)$ f 2.81( 29.87\%)g $0.00(\quad 0.00 \%)$ \\ ( $85.66 \%) \quad 0.9255 * \mathrm{Cl} \quad 5 \mathrm{~s}(36.74 \%) \mathrm{p} 1.72(63.26 \%)$
}


11.BD ( 1) U 2 -Cl 6

$(13.23 \%) \quad 0.3638 * \mathrm{U} \quad 2 \mathrm{~s}(10.21 \%)$ p $1.95(19.86 \%) d 4.08(41.70 \%) f 2.76(28.22 \%) g \quad 0.00(\quad 0.01 \%)$

$(86.77 \%) \quad 0.9315 * \mathrm{Cl} \quad 6 \mathrm{~s}(36.86 \%) \mathrm{p} 1.71(63.14 \%)$

\section{BD ( 1) U 2 -Cl 11}

( $16.83 \%) \quad 0.4103 * \mathrm{U} \quad 2 \mathrm{~s}(19.16 \%) \mathrm{p} 1.18(22.59 \%) \mathrm{d} 2.00(38.30 \%)$ f $1.04(19.94 \%) \mathrm{g} 0.00(\quad 0.01 \%)$

( 83.17\%) $\quad 0.9120 * \mathrm{Cl} \quad 11 \mathrm{~s}(56.16 \%) \mathrm{p} \mathrm{0.78(43.84 \% )}$

13.BD ( 1) U 2 - P 12

$(17.76 \%) \quad 0.4214 *$ U $\quad 2$ s( $\quad 9.92 \%)$ p $2.46(24.37 \%)$ d $3.79(37.61 \%)$ f $2.83(28.10 \%)$ g $0.00(\quad 0.00 \%)$

$(82.24 \%) \quad 0.9069 *$ P 12 s( $41.37 \%)$ p $1.42(58.56 \%) d 0.00(\quad 0.07 \%)$

\section{BD ( 1) U 2 - N 16}

( 9.22\%) $\quad 0.3037 * \mathrm{U} \quad 2$ s( $13.46 \%)$ p $1.10(14.81 \%) d 2.47(33.19 \%)$ f 2.86(38.53\%)g $0.00(\quad 0.00 \%)$

$(90.78 \%) \quad 0.9528 * N \quad 16$ s( $25.48 \%)$ p $2.92(74.50 \%) d 0.00(\quad 0.01 \%)$

15.BD ( 1) U 2 - N 17

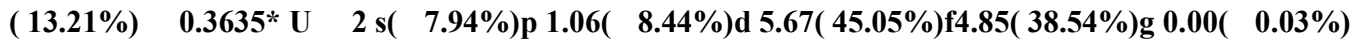

( 86.79\%) $\quad 0.9316 * \mathrm{~N} \quad 17$ s( $47.37 \%)$ p 1.11( $52.62 \%) d$ 0.00( $0.01 \%)$

\section{BD (2) U 2 - N 17}

$\begin{array}{llll}(11.89 \%) & 0.3448 * \mathrm{U} & 2 \mathrm{~s}(\quad \mathbf{0 . 4 8} \%) \text { p16.50( } 7.91 \%) d 91.08(43.67 \%) f 99.93(47.92 \%) g & 0.03(\quad 0.01 \%)\end{array}$

$(88.11 \%) \quad 0.9387 * \mathrm{~N} \quad 17 \mathrm{~s}(\quad \mathbf{0 . 0 5} \%)$ p99.99( $99.93 \%)$ d $0.25(\quad 0.01 \%)$

\section{BD ( 1) U 2 - N 18}

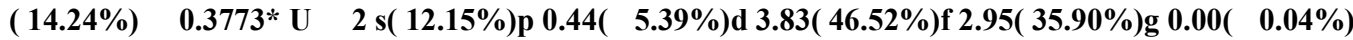

$(85.76 \%) \quad 0.9261 * \mathrm{~N} \quad 18$ s( $46.81 \%) p 1.14(53.18 \%) d 0.00(\quad 0.01 \%)$

\section{BD ( 2) U 2 - N 18}

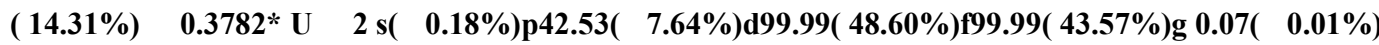
$(85.69 \%) \quad 0.9257 * N \quad 18$ s( $\quad 0.00 \%) p 1.00(99.99 \%) d 0.00(\quad 0.01 \%)$

\begin{tabular}{|c|c|c|}
\hline \multicolumn{3}{|c|}{ Donation } \\
\hline From & To & Energy (kcal/mol) \\
\hline Cr1 U1 & Rh & 8.36 \\
\hline CR2 U1 & Rh & 33.5 \\
\hline CR4 U1 & Rh & 4.84 \\
\hline CR6 U1 & Rh & 1.54 \\
\hline CR8 U1 & Rh & 0.7 \\
\hline Cr1 U2 & Rh & 12.44 \\
\hline CR2 U2 & Rh & 58.44 \\
\hline CR4 U2 & Rh & 8.54 \\
\hline
\end{tabular}




\begin{tabular}{|c|c|c|}
\hline CR6 U2 & Rh & 1.84 \\
\hline CR8 U2 & Rh & 6.88 \\
\hline Cr1 Rh & U1 & 46.34 \\
\hline Cr1 Rh & U2 & 40.94 \\
\hline Cr2 Rh & U1 & 5.16 \\
\hline Cr2 Rh & U2 & 3.04 \\
\hline Cr3 Rh & U1 & 0.78 \\
\hline Cr3 Rh & U2 & 0.7 \\
\hline Cr4 Rh & U1 & 5.34 \\
\hline Cr4 Rh & U2 & 4.36 \\
\hline LP1 Rh & U1 & 7.6 \\
\hline LP1 Rh & U2 & 6.52 \\
\hline LP2 Rh & U1 & 1.3 \\
\hline LP3 Rh & U1 & 44.12 \\
\hline LP3 Rh & U2 & 30.52 \\
\hline LP4 $\mathbf{R h}$ & U1 & 10.26 \\
\hline
\end{tabular}

Spin density

$$
\mathrm{U} 1=\mathbf{2 . 1 6 0 3 0 8} \mathrm{U} 2=\mathbf{2 . 1 5 9 8 9 6} \quad \mathrm{Rh}=\mathbf{- 0 . 0 1 6 4 9 2}
$$

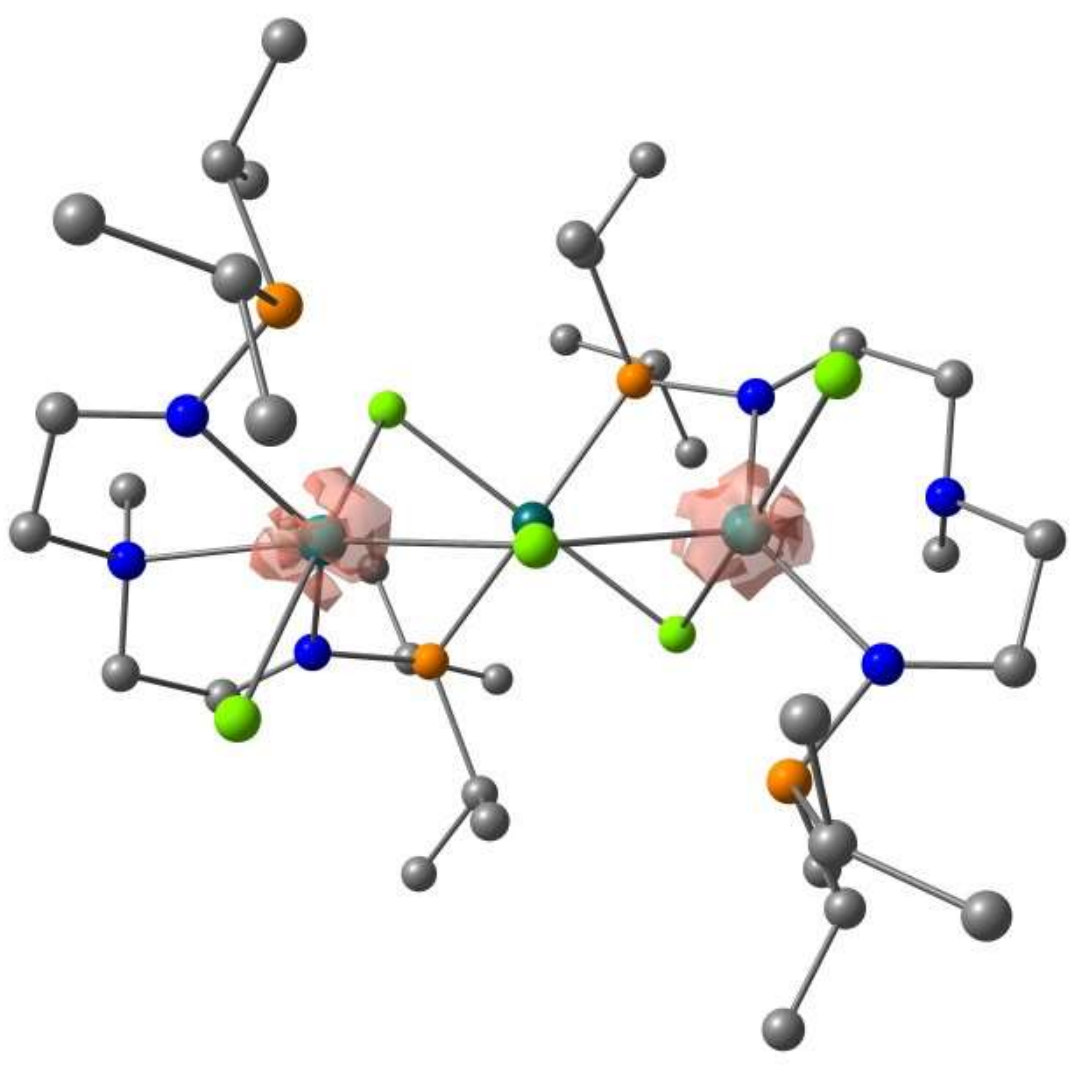




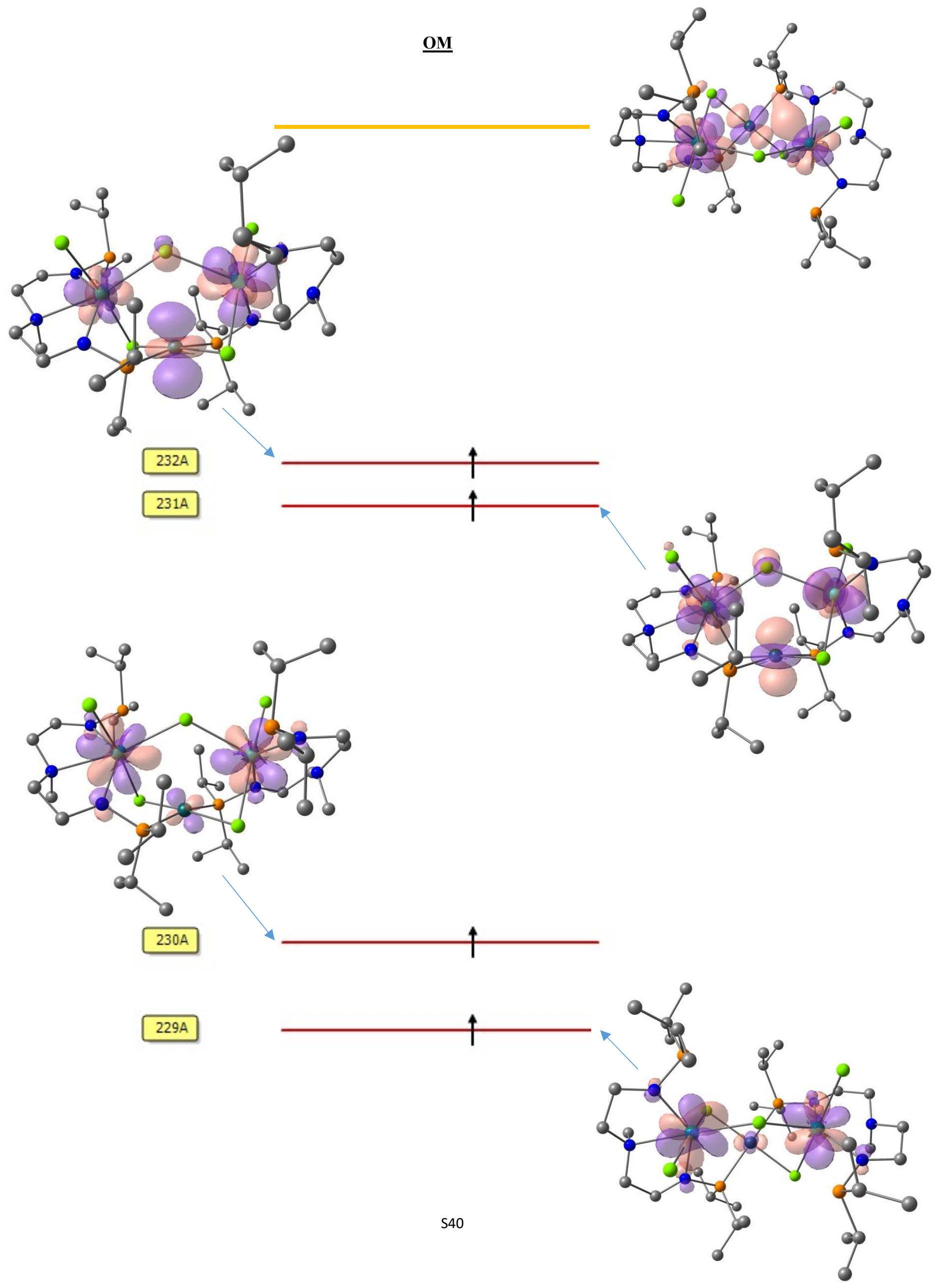




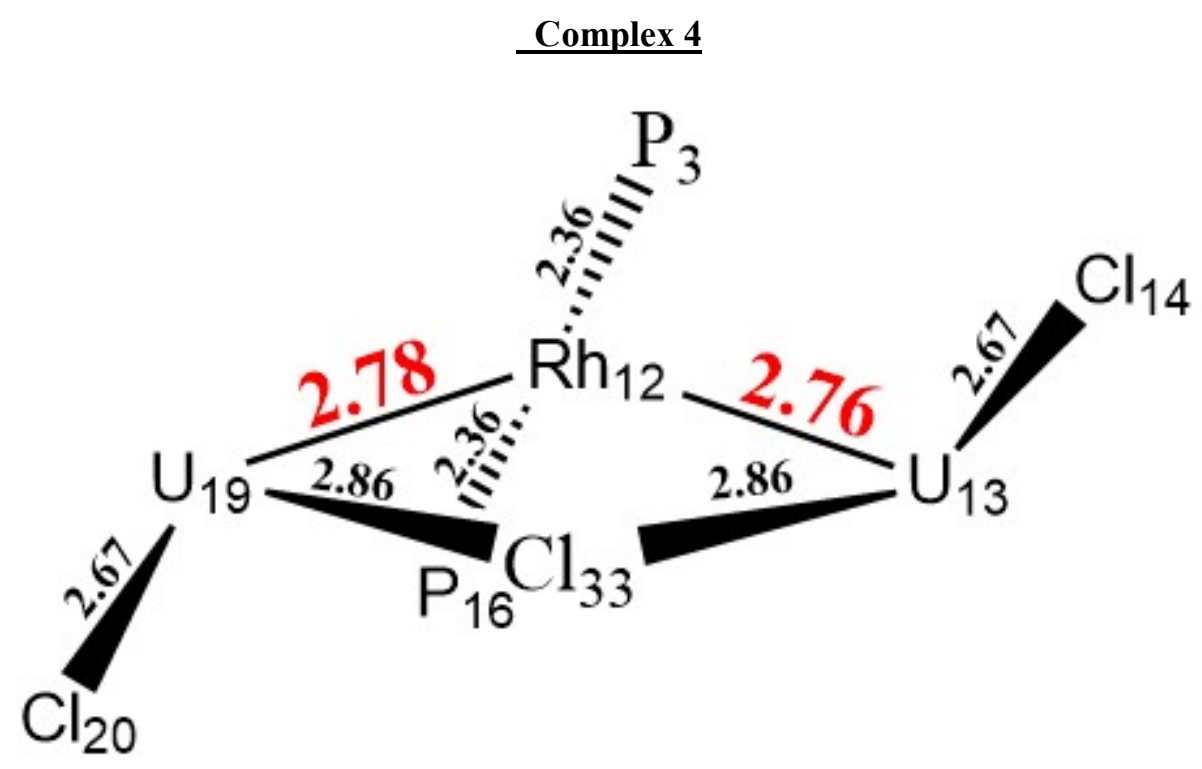

Natural charge

\begin{tabular}{|l|l|l|l|ll|}
\hline $\mathbf{U}_{\mathbf{1 3}}=0,80529$ & $\mathbf{U}_{\mathbf{1 9}}=0,81708$ & $\mathbf{R h}_{\mathbf{1 2}}=-0,77027$ & $\mathbf{C l}_{\mathbf{1 4}}=0,31741$ \\
\hline $\mathbf{C l}_{\mathbf{2 0}}=0,31796$ & $\mathbf{C l}_{\mathbf{3 3}}=-0,17941$ & $\mathbf{P}_{\mathbf{3}}=1,43214$ & $\mathbf{P}_{\mathbf{1 6}}=1,42891$ \\
\hline
\end{tabular}

\section{$\underline{\text { Natural Electron Configuration }}$}

$\mathbf{U}_{13} \quad[$ core $] 7 \mathrm{~S}(0,19) 5 \mathrm{f}(2,89) 6 \mathrm{~d}(1,53) 7 \mathrm{p}(0,39)$

$\mathbf{U}_{19} \quad$ [core]7S( 0,19$) 5 \mathrm{f}(2,88) 6 \mathrm{~d}(1,52) 7 \mathrm{p}(0,39)$

$\mathbf{R h}_{12} \quad$ core]5S( 0,51)4d( 9,04)5p(0,27)5d(0,01)6p( 0,01)6d( 0,01)

\section{Wiberg bond index}

$$
\begin{aligned}
& \mathbf{U}_{\mathbf{1 3}}-\mathbf{R h}=0,8401 \quad \mathbf{U}_{\mathbf{1 3}}-\mathbf{C l}_{\mathbf{1 4}}=1,0937 \quad \mathbf{U}_{\mathbf{1 3}}-\mathbf{C l}_{\mathbf{3 3}}=0,6345 \\
& \mathbf{U}_{\mathbf{1 9}}-\mathbf{R h}=0,8261 \quad \mathbf{U}_{\mathbf{1 9}}-\mathbf{C l}_{\mathbf{2 0}}=1,0933 \quad \mathbf{U}_{\mathbf{1 9}}-\mathbf{C l}_{\mathbf{3 3}}=0,6365 \\
& \mathbf{U}_{\mathbf{1 3}}-\mathbf{U}_{\mathbf{1 9}}=0,01517 \\
& \mathbf{R h}-\mathbf{P h}_{\mathbf{3}}=0,3914 \quad \mathbf{R h}-\mathbf{P h}_{16}=0,3886
\end{aligned}
$$

Bond

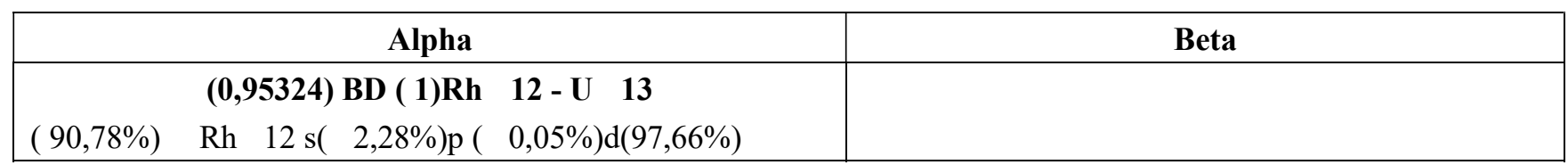




\begin{tabular}{|c|c|}
\hline U $13 \mathrm{~s}(3,32 \%) p(11,24 \%) d(35,14 \%) f(50,29 \%)$ & \\
\hline $\begin{array}{lcl} & \mathbf{( 0 , 9 5 0 8 6 )} \text { BD ( 1)Rh } & \mathbf{1 2}-\mathbf{U} \quad \mathbf{1 9} \\
(90,81 \%) & \mathrm{Rh} 12 \mathrm{~s}(\quad 2,56 \%) \mathrm{p}(\quad 0,06 \%) \mathrm{d}(97,38 \%) \\
(9,19 \%) & \mathrm{U} \quad 19 \mathrm{~s}(3,86 \%) \mathrm{p}(11,94 \%) \mathrm{d}(35,29 \%) \mathrm{f}(48,91 \%)\end{array}$ & $\begin{array}{l}\text { (0,93294) BD ( 1)Rh } 12-\text { U } 19 \\
(94,38 \%) \text { Rh } 12 \mathrm{~s}(\quad 4,87 \%) p(\quad 0,04 \%) d(95,08 \%) \\
(5,62 \%) \text { U } 19 \\
\text { s( } 13,13 \%) p(25,64 \%) d(32,96 \%) f(28,25 \%)\end{array}$ \\
\hline $\begin{array}{ll} & \text { (0,97816) BD ( 1) U } \quad \mathbf{1 3} \text {-Cl } \mathbf{1 4} \\
(14,61 \%) & \mathrm{U} 13 \mathrm{~s}(21,18 \%) \mathrm{p}(21,59 \%) \mathrm{d}(38,60 \%) \mathrm{f}(18,62 \%) \\
(85,39 \%) & \mathrm{C} 114 \mathrm{~s}(51,41 \%) \mathrm{p}(48,59 \%)\end{array}$ & $\begin{array}{l}\text { (0,98230) BD ( 1) U } \quad \mathbf{1 3} \text {-Cl } 14 \\
(14,45 \%) \quad \mathrm{U} \quad 13 \\
\mathrm{~s}(22,65 \%) \mathrm{p}(16,75 \%) \mathrm{d}(46,41 \%) \mathrm{f}(14,17 \%) \\
(85,55 \%) \quad \mathrm{Cl} \quad 14 \mathrm{~s}(55,36 \%) \mathrm{p}(44,64 \%)\end{array}$ \\
\hline 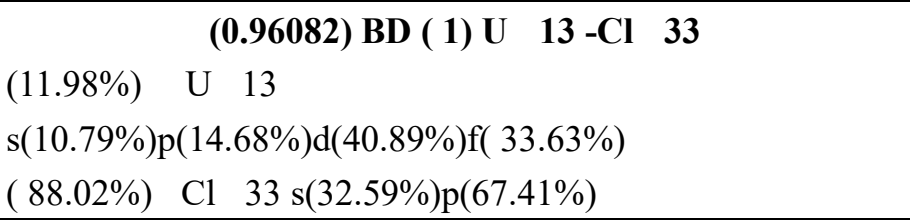 & $\begin{array}{l}\text { (0,96070) BD ( 1) U } \quad 13 \text {-Cl } 33 \\
(11,55 \%) \quad \mathrm{U} \quad 13 \\
\mathrm{~s}(10,12 \%) \mathrm{p}(16,24 \%) \mathrm{d}(38,94 \%) \mathrm{f}(34,69 \%) \\
(88,45 \%) \quad \mathrm{Cl} 33 \mathrm{~s}(34,06 \%) \mathrm{p}(65,94 \%) \\
\end{array}$ \\
\hline $\begin{array}{ll} & \mathbf{( 0 , 9 7 8 0 9 )} \text { BD ( 1) U } \quad \mathbf{1 9} \text {-Cl } \mathbf{2 0} \\
(14,60 \%) & \mathrm{U} 19 \mathrm{~s}(21,01 \%) \mathrm{p}(21,56 \%) \mathrm{d}(38,44 \%) \mathrm{f}(18,98 \%) \\
(85,40 \%) & \mathrm{Cl} 20 \mathrm{~s}(51,07 \%) \mathrm{p}(48,93 \%)\end{array}$ & $\begin{array}{l}\text { (0,97834) BD ( 1) U } 19 \text {-Cl } 20 \\
(13,92 \%) \quad \text { U } 19 \\
s(17,00 \%) p(26,99 \%) d(36,64 \%) f(19,36 \%) \\
(86,08 \%) \quad \text { Cl } 20 \mathrm{~s}(55,15 \%) p(44,85 \%)\end{array}$ \\
\hline $\begin{array}{l}\text { (0,96059) BD ( 1) U } \quad \text { 19-Cl } 33 \\
(11,96 \%) \\
\text { U19 } \\
s(10,93 \%) p(14,45 \%) d(41,03 \%) f(33,58 \%) \\
(88,04 \%) \quad \text { Cl33 s( } 32,41 \%) p(67,59 \%)\end{array}$ & $\begin{array}{l}\text { (0,96060) BD ( 1) U } \quad \mathbf{1 9} \text {-Cl } 33 \\
(11,50 \%) \quad \mathrm{U} \quad 19 \\
\mathrm{~s}(11,26 \%) \mathrm{p}(15,50 \%) \mathrm{d}(38,28 \%) \mathrm{f}(34,95 \%) \\
(88,50 \%) \quad \mathrm{Cl} 33 \mathrm{~s}(34,02 \%) \mathrm{p}(65,98 \%)\end{array}$ \\
\hline
\end{tabular}

Alpha donation:

\begin{tabular}{|c|c|c|}
\hline Donor & Acceptor & 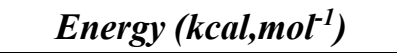 \\
\hline$U 13$ & \multirow{2}{*}{$\boldsymbol{R h}$} & $44,73(83 \% C R-17 \% L P)$ \\
\hline$U 19$ & & $44,29(82 \% C R-18 \% L P)$ \\
\hline $\boldsymbol{U}$ & $U$ & $23,52(75 \% C R-25 \% L P)$ \\
\hline $\boldsymbol{R h}$ & $U 13$ & $57,02(96 \% C R-4 \% L P)$ \\
\hline $\boldsymbol{R h}$ & $U 19$ & $54,17(95 \% C R-5 \% L P)$ \\
\hline
\end{tabular}




\section{Orbitals}

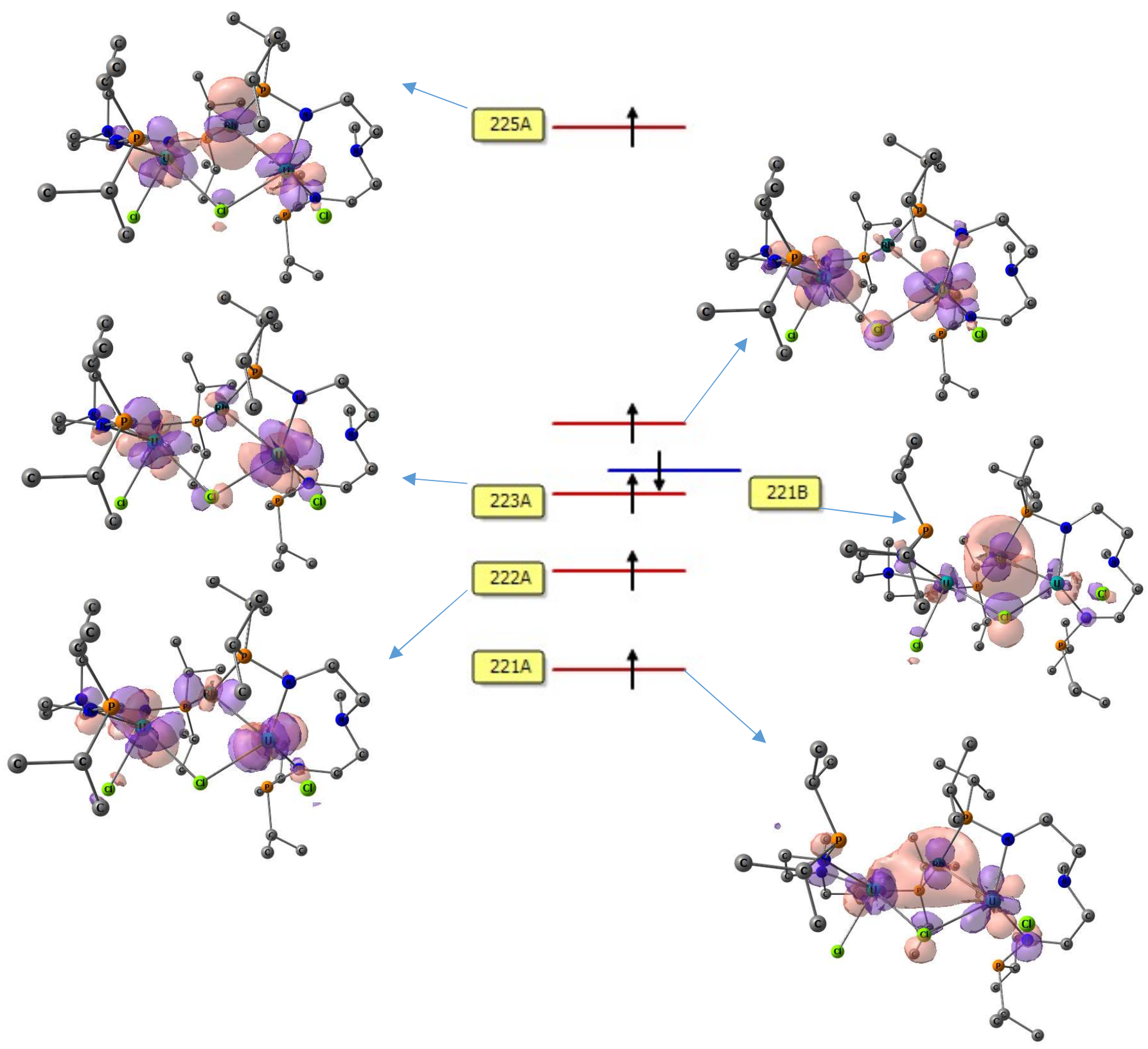

\begin{tabular}{|l|l|l|l|l|l|}
\hline & Exp. & Cal. & & Exp. & Cal. \\
\hline U1-Rh1 & $2.6555(6)$ & 2.77 & U1-N1 & $2.310(6)$ & \\
\hline U1-C11 & $2.8055(18)$ & 2.86 & U1-N2 & $2.255(6)$ & \\
\hline U1-C12 & $2.6438(19)$ & 2.67 & U1-N3 & $2.642(6)$ & \\
\hline U1-P1 & $3.1256(19)$ & & Rh1-P1 & $2.370(2)$ & 2.36 \\
\hline U1-P2 & $3.000(2)$ & & U1---U1 & $4.0397(6)$ & \\
\hline
\end{tabular}




\section{Complex 4 with dispersion}

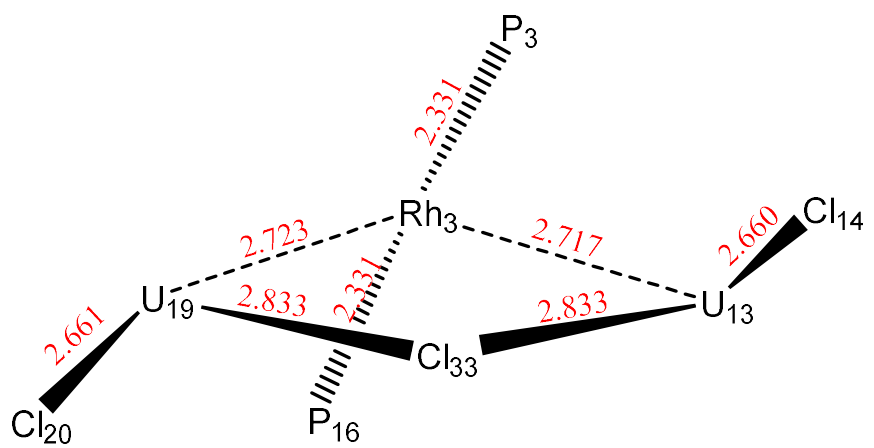

Natural Charge

\begin{tabular}{|c|c|l|l|}
\hline $\mathbf{U}_{13}=$ & $\mathbf{U}_{19}=$ & $\mathbf{R h}_{3}=-0.78081$ & $\mathbf{C l}_{14}=-$ \\
0.64740 & 0.65076 & & 0.29834 \\
\hline $\mathbf{C l}_{\mathbf{2 0}}=-0.29930$ & $\begin{array}{c}\mathbf{C l}_{33}=- \\
0.13269\end{array}$ & $\mathbf{P}_{3}=1.47806$ & $\begin{array}{c}\mathbf{P}_{16}= \\
1.47718\end{array}$ \\
\hline
\end{tabular}

\section{Natural Electron Configuration}

U $\quad 13 \quad[$ core $7 \mathrm{~S}(0.19) 5 \mathrm{f}(2.93) 6 \mathrm{~d}(1.60) 7 \mathrm{p}(0.41)$

U $19 \quad$ [core]7S( 0.19)5f( 2.93)6d( 1.60)7p( 0.41)

$\mathrm{Rh} \quad \quad[$ core $5 \mathrm{~S}(0.51) 4 \mathrm{~d}(9.04) 5 \mathrm{p}(0.29) 5 \mathrm{~d}(0.01) 6 \mathrm{p}(0.01) 6 \mathrm{~d}(0.01)$

\section{Wiberg bond index}

$$
\begin{aligned}
& \mathbf{U}_{13}-\mathbf{R h}=0.8541 \mathbf{U}_{\mathbf{1 3}}-\mathbf{C l}_{\mathbf{1} \mathbf{4}}=1.1157 \quad \mathbf{U}_{\mathbf{1 3}}-\mathbf{C l}_{\mathbf{3} 3}=0.6638 \\
& \mathbf{U}_{19}-\mathbf{R h}=0.8454 \quad \mathbf{U}_{19}-\mathbf{C l}_{20}=1.1139 \quad \mathbf{U}_{19}-\mathbf{C l}_{33}=0.6626 \\
& \mathrm{U}_{13}-\mathrm{U}_{19}=0.1703
\end{aligned}
$$

$$
\mathbf{R h}-\mathbf{P h}_{3}=0.3887 \quad \mathbf{R h}-\mathbf{P h}_{16}=0.3878
$$

\section{Total Wiberg Index}

\begin{tabular}{c|c|c|c}
$\mathbf{U}_{13}=5.9733$ & $\mathbf{U}_{19}=5.9668$ & $\mathbf{R h}=3.1129$
\end{tabular}


54. (0.97633) BD ( 1) U 19 -Cl 20

$(14.93 \%) \quad 0.3863 * \mathrm{U} \quad 19 \mathrm{~s}(19.69 \%) \mathrm{p} 1.23(24.26 \%) \mathrm{d} .02(39.81 \%)$ f $0.82(16.21 \%) \mathrm{0.00}(\quad 0.02 \%)$

( $85.07 \%) \quad 0.9224 * \mathrm{Cl} \quad 20$ s( $53.39 \%)$ p $0.87(46.61 \%)$

55. (0.96791) BD ( 1) U 19 - N 23

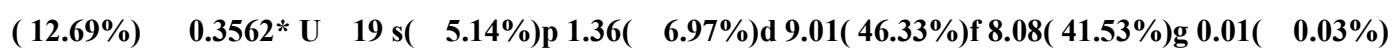

( 87.31\%) $\quad 0.9344 * N \quad 23$ s( $46.00 \%) p 1.17(53.99 \%) d ~ 0.00(\quad 0.01 \%)$

56. (0.93065) BD ( 2) U 19 - N 23

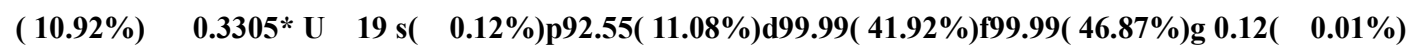

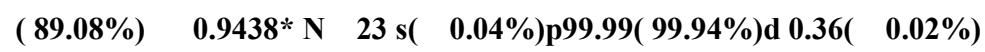

57. (0.94833) BD ( 1) U 19 - N 26

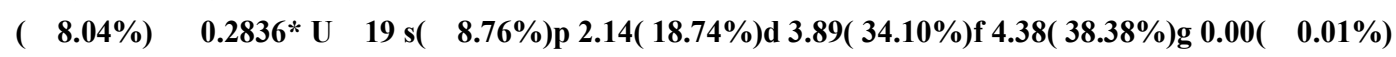
$(91.96 \%) \quad 0.9589^{*} \mathrm{~N} \quad 26 \mathrm{~s}(23.00 \%) \mathrm{s} 3.35(76.99 \%) \mathrm{d} 0.00(\quad 0.01 \%)$

58. (0.96926) BD ( 1) U 19 - N 29

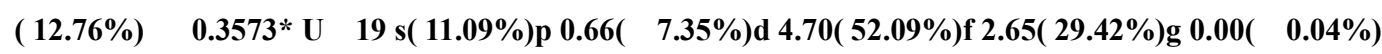

$(87.24 \%) \quad 0.9340 * \mathrm{~N} \quad 29 \mathrm{~s}(45.45 \%) p 1.20(54.54 \%) d 0.00(\quad 0.01 \%)$

59. (0.93330) BD ( 2) U 19 - N 29

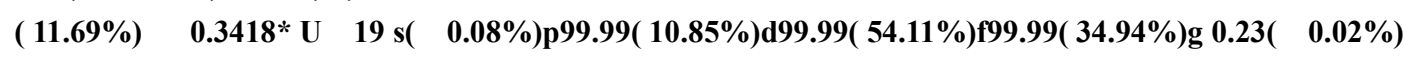

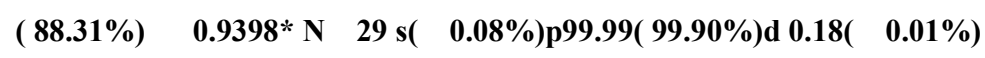

60. (0.92119) BD ( 1) U 19 - P 30

( $14.03 \%) \quad 0.3746 *$ U 19 s( $11.25 \%)$ p 2.64( 29.73\%)d 3.27( 36.82\%)f $1.97(22.20 \%)$ g $0.00(\quad 0.00 \%)$

$(85.97 \%) \quad 0.9272 * \mathrm{P} \quad 30 \mathrm{~s}(41.69 \%) \mathrm{p} 1.40(58.27 \%) \mathrm{d}$ 0.00( $\quad 0.04 \%)$

61. (0.95592) BD ( 1) U 19 -Cl 33

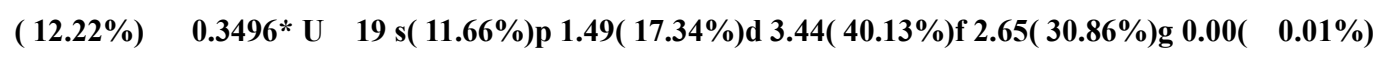

$(87.78 \%) \quad 0.9369 *$ Cl 33 s( 32.89\%)p 2.04( 67.11\%)

\begin{tabular}{|c|c|c|}
\hline \multicolumn{3}{|c|}{ Donation } \\
\hline From & To & Energy (kcal/mol) \\
\hline CR1 N4 & U13 & 1,18 \\
\hline Cr1 Rh & U13 & 98,4 \\
\hline Cr2 Rh & U13 & 0,98 \\
\hline Cr3 Rh & U13 & 3,08 \\
\hline Cr4 Rh & U13 & 5,28 \\
\hline Cr1 Rh & U19 & 79,32 \\
\hline Cr2 Rh & U19 & 1,14 \\
\hline Cr3 Rh & U19 & 2,7 \\
\hline
\end{tabular}




\begin{tabular}{|c|c|c|}
\hline Cr4 Rh & U19 & 1,66 \\
\hline Cr1 U13 & Rh & 16,12 \\
\hline Cr2 U13 & Rh & 64,92 \\
\hline Cr6 U13 & Rh & 2,54 \\
\hline Cr8 U13 & Rh & 6,26 \\
\hline Cr1 U19 & Rh & 16,06 \\
\hline Cr2 U19 & Rh & 66,32 \\
\hline Cr4 U19 & Rh & 6,98 \\
\hline Cr6 U19 & Rh & 0,68 \\
\hline Cr8 U19 & Rh & 2,88 \\
\hline LP1 P3 & U13 & 39,68 \\
\hline LP1 P3 & U19 & 10,3 \\
\hline LP1 Rh & U13 & 4,06 \\
\hline LP1 Rh & U19 & 1,9 \\
\hline LP2 Rh & U13 & 3,86 \\
\hline LP2 Rh & U19 & 2,1 \\
\hline LP2 U13 & Rh & 7,04 \\
\hline LP1 P16 & U13 & 11,72 \\
\hline LP1 P16 & U19 & 23,92 \\
\hline
\end{tabular}

Spin density

$$
\mathrm{U} 13=2.202294 \mathrm{U} 19=\mathbf{2 . 2 0 2 1 9 1} \mathrm{Rh}=\mathbf{- 0 . 1 4 3 5 8 6}
$$

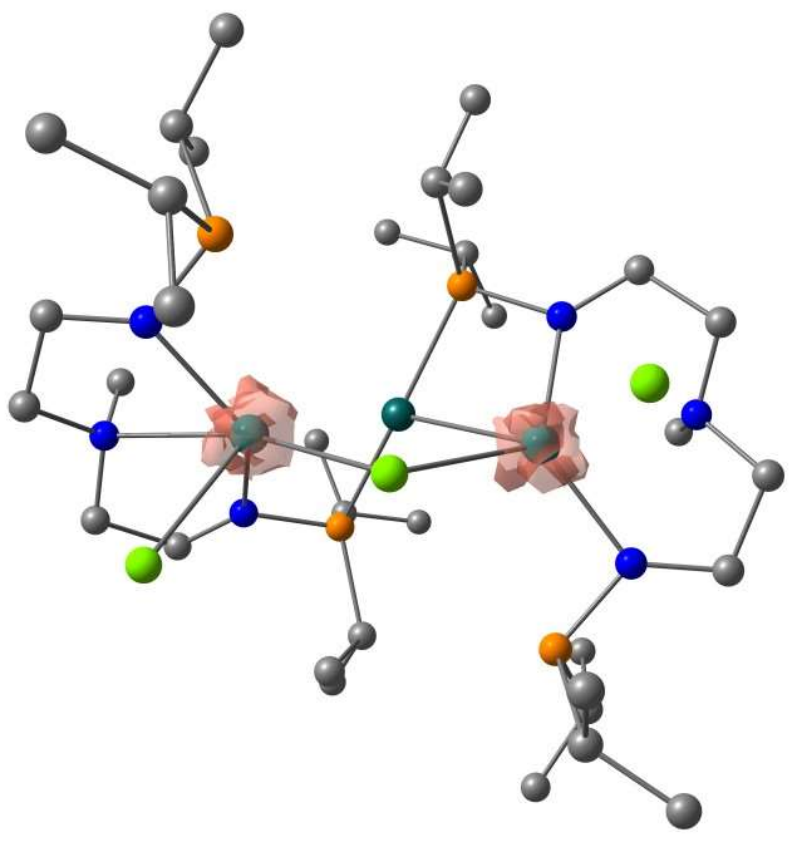


$\underline{O M}$
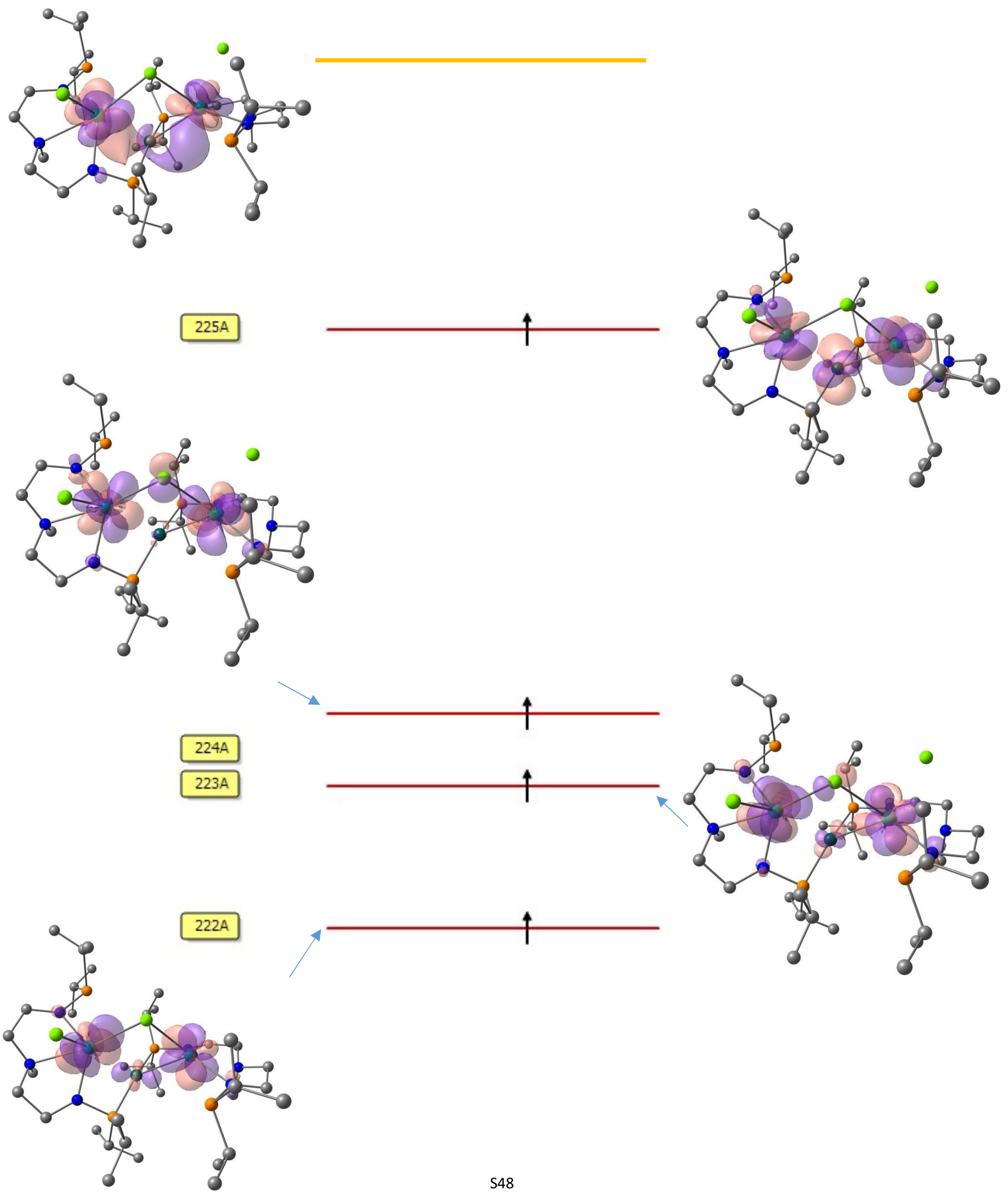

$224 \mathrm{~A}$

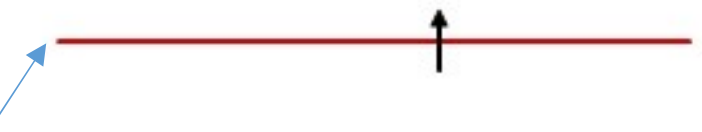




\section{Complex 5}
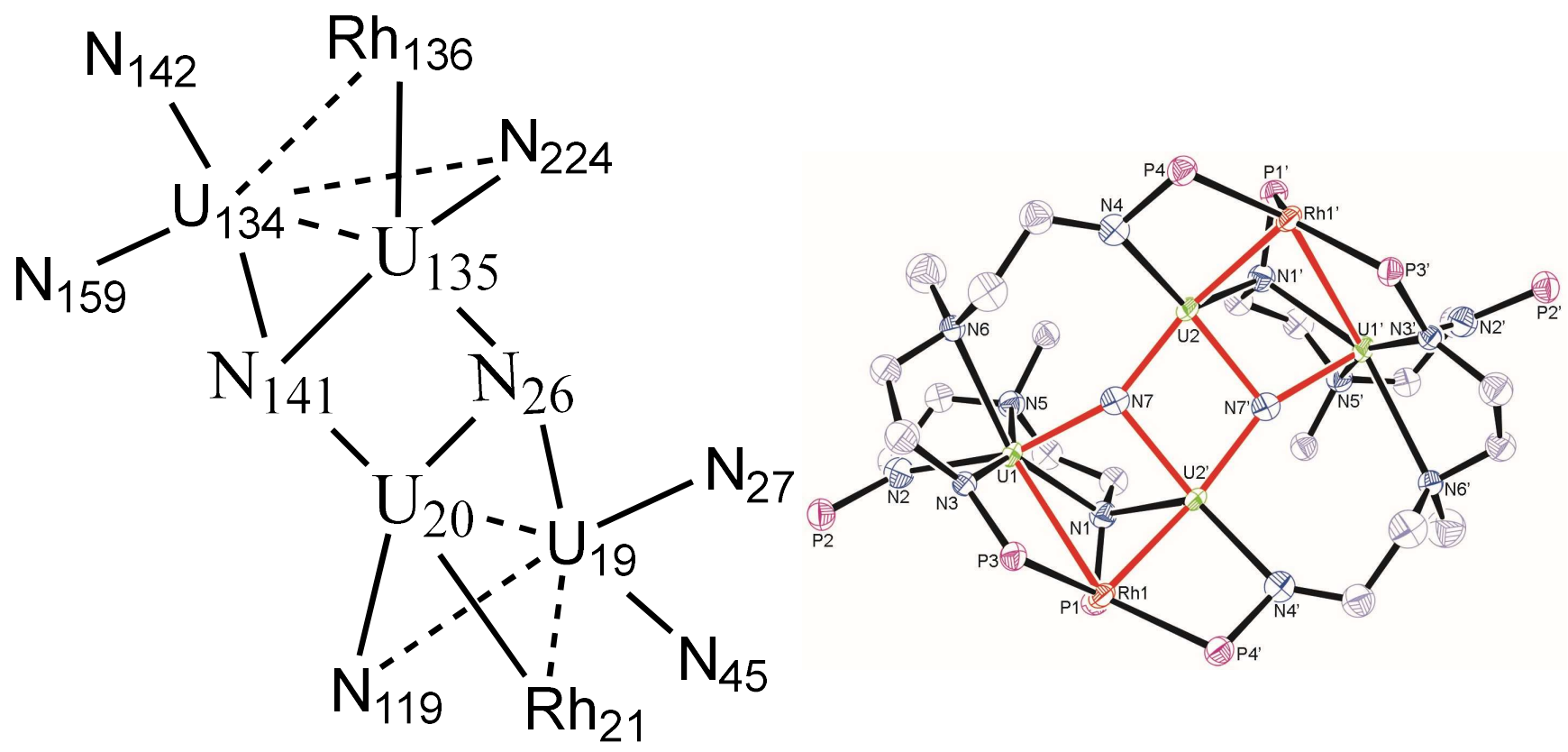

Distances

\begin{tabular}{|l|l|l|l|l|l|}
\hline & Exp. & Cal. & & Exp. & Cal. \\
\hline U1---U2' & $3.4677(4)$ & 3.53 & U2---U2' & $3.4730(6)$ & 3.53 \\
\hline U1-Rh1 & $3.2160(7)$ & 3.23 & U2-N4 & $2.371(7)$ & 2.42 \\
\hline U2'-Rh1 & $2.5139(7)$ & 2.80 & U2-N1' & $2.605(7)$ & 2.60 \\
\hline U1-N3 & $2.282(7)$ & 2.34 & U2-N7' & $2.302(6)$ & 2.30 \\
\hline U1-N6 & $2.847(7)$ & 2.84 & U2-N7 & $2.154(7)$ & 2.25 \\
\hline U1-N2 & $2.461(7)$ & 2.54 & Rh1'-P1' & $2.333(2)$ & 2.34 \\
\hline U1-N1 & $2.552(6)$ & 2.65 & Rh1'-P4 & $2.438(2)$ & 2.45 \\
\hline U1-N5 & $2.732(7)$ & 2.88 & Rh1-P1 & $2.333(2)$ & 2.34 \\
\hline U1-N7 & $2.158(7)$ & 2.24 & U2'-N7 & $2.302(6)$ & 2.25 \\
\hline U1-P3 & $3.203(2)$ & 3.30 & U2-P4 & $3.120(2)$ & 3.25 \\
\hline U1-P1 & $3.384(2)$ & 3.46 & U2-P1' & $3.135(2)$ & 2.81 \\
\hline
\end{tabular}

Natural Charge

\begin{tabular}{|l|c|c|c|}
\hline $\mathrm{U}_{19}=2.19033$ & $\mathrm{U}_{20}=2.14570$ & $\mathrm{U}_{134}=2.19036$ & $\mathrm{U}_{135}=2.14578$ \\
\hline $\mathrm{Rh}_{21}=-1.23278$ & $\mathrm{Rh}_{136}=-1.23283$ & $\mathrm{~N}_{26}=-1.82873$ & $\mathrm{~N}_{27}=-1.31692$ \\
\hline $\mathrm{N}_{45}=-1.23630$ & $\mathrm{~N}_{119}=-1.27271$ & $\mathrm{~N}_{141}=-1.82875$ & $\mathrm{~N}_{142}=-1.31693$ \\
\hline $\mathrm{N}_{224}=-1.27272$ & & & \\
\hline
\end{tabular}




\begin{tabular}{|c|c|}
\hline \multicolumn{2}{|c|}{ Small Core Unpaired Spin density } \\
\hline U1 & 2.04 \\
\hline U2 & 2.03 \\
\hline U3 & 2.02 \\
\hline U4 & 2.05 \\
\hline
\end{tabular}

\section{Natural Electron Configuration}

$\mathbf{U}_{19} \quad[$ core]7S( 1.96)5f( 0.01)6d( 1.32)7p( 0.42)

$\mathbf{U}_{\mathbf{2 0}} \quad$ [core]7S( 1.93)5f( 0.01)6d( 1.48)7p(0.39)

$\mathbf{U}_{134} \quad$ [core]7S( 1.96)5f( 0.01)6d( 1.32)7p( 0.42)

$\mathbf{U}_{135} \quad$ [core]7S( 1.93)5f( 0.01)6d( 1.48)7p( 0.39)

$\mathbf{R h}_{21} \quad$ [core]5S( 0.43)4d( 9.19)5p( 0.62)5d( 0.01)6p( 0.01)6d( 0.02)

$\mathbf{R h}_{136} \quad$ [core]5S( 0.43)4d( 9.19)5p( 0.62)5d( 0.01)6p( 0.01)6d( 0.02)

$\mathbf{N}_{26} \quad$ [core]2S( 1.68)2p( 5.15)

$\mathbf{N}_{27} \quad$ [core $] 2 S(1.46) 2 \mathrm{p}(4.85) 3 \mathrm{p}(0.01)$

$\mathbf{N}_{45} \quad$ [core $2 S(1.48) 2 \mathrm{p}(4.74) 3 \mathrm{p}(0.01)$

$\mathbf{N}_{119} \quad$ [core]2S( 1.45)2p( 4.81)3p( 0.01)

$\mathbf{N}_{141} \quad$ [core]2S( 1.68)2p( 5.15)

$\mathbf{N}_{142} \quad$ [core]2S( 1.46)2p( 4.85)3p( 0.01)

$\mathbf{N}_{224} \quad$ [core]2S( 1.45)2p( 4.81)3p( 0.01)

\section{Wiberg Bond Indexes}

\begin{tabular}{|c|c|c|c|c|}
\hline U19-Rh21 $=\mathbf{0 . 3 5 3 9}$ & U19-N26 $=\mathbf{0 . 5 3 8 6}$ & U19-N27 $=\mathbf{0 . 3 3 7 8}$ & U19-N45 $=\mathbf{0 . 3 6 1 8}$ & U19-N119 $=\mathbf{0 . 2 2 1 6}$ \\
\hline U20-U19 $=\mathbf{0 . 2 4 9 8}$ & U20-Rh21 $=\mathbf{0 . 5 2 0 2}$ & U20-N26 $=\mathbf{0 . 5 5 6 0}$ & U20-N141 $=\mathbf{0 . 6 0 8 0}$ & U20-N119 $=\mathbf{0 . 2 3 3 6}$ \\
\hline U134-U135 $=\mathbf{0 . 2 4 9 8}$ & U134-Rh136 $=\mathbf{0 . 3 5 4 0}$ & U134-N141 $=\mathbf{0 . 5 3 8 6}$ & U134-N224 $=\mathbf{0 . 2 2 1 6}$ & U134-N142 $=\mathbf{0 . 3 3 7 8}$ \\
\hline U134-N159 $=\mathbf{0 . 3 6 1 8}$ & U135-N26 $=\mathbf{0 . 6 0 8 0}$ & U135-N141 $=\mathbf{0 . 5 5 6 0}$ & U135-Rh136 $=\mathbf{0 . 5 2 0 1}$ & U135-N224 = 0.2336 \\
\hline
\end{tabular}

Bond

20. (1.65361) BD ( 1) U 20 - P 24 
$0.99(0.08 \%)$

$(10.14 \%) \quad 0.3185 * \mathrm{U} \quad 20 \mathrm{~s}(\quad 0.08 \%) \mathrm{p} 99.99(21.72 \%) \mathrm{d} 99.99(78.11 \%) \mathrm{f}$

$(89.86 \%) \quad 0.9479 * \mathrm{P} \quad 24 \mathrm{~s}(45.31 \%) \mathrm{p} 1.21(54.67 \%) \mathrm{d} 0.00(\quad 0.02 \%)$

35. (1.71642) BD ( 1) N 26 - U 135

$9.65(\quad 0.55 \%)$

$\begin{array}{lll}(91.13 \%) & 0.9546 * \mathrm{~N} \quad 26 \mathrm{~s}(\quad 0.04 \%) \mathrm{p} 99.99(99.95 \%) \mathrm{d} 0.22(\quad 0.01 \%)\end{array}$

( $8.87 \%) \quad 0.2979 *$ U $135 \mathrm{~s}(\quad 0.06 \%) \mathrm{p} 99.99(12.27 \%) \mathrm{d} 99.99(87.12 \%) \mathrm{f}$

139. (1.64282) BD ( 1) U 135 - P 139

( $9.28 \%) \quad 0.3046 *$ U $135 \mathrm{~s}(\quad 0.14 \%) p 99.99(34.95 \%) \mathrm{d} 99.99(64.74 \%)$

$1.24(\quad 0.17 \%)$

( 90.72\%) $\quad 0.9525 *$ P $139 \mathrm{~s}(45.31 \%)$ p $1.21(54.67 \%) \mathrm{d} 0.00(\quad 0.02 \%)$

140. (1.73275) BD ( 1) U 135 - N 141

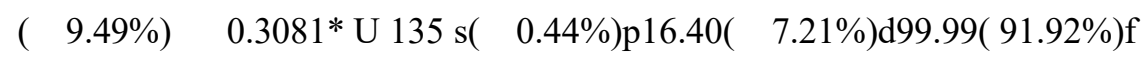

$0.98(0.43 \%)$

$(90.51 \%) \quad 0.9514 * \mathrm{~N} 141 \mathrm{~s}(\quad 0.05 \%) \mathrm{p} 99.99(99.95 \%) \mathrm{d} 0.20(\quad 0.01 \%)$

21. (1.67796) BD ( 1)Rh 21 - P 22

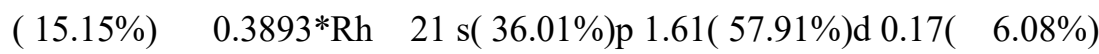

$(84.85 \%) \quad 0.9211 * \mathrm{P} \quad 22 \mathrm{~s}(49.58 \%) \mathrm{p} 1.02(50.39 \%) \mathrm{d} 0.00(\quad 0.04 \%)$

22. (1.67664) BD (1)Rh 21 - P 23

(17.63\%) $\quad 0.4199 *$ Rh $\quad 21 \mathrm{~s}(39.93 \%) p$ 1.31( 52.12\%)d 0.20( $7.95 \%)$

( 82.37\%) $\quad 0.9076 * \mathrm{P} \quad 23 \mathrm{~s}(42.47 \%) \mathrm{p} 1.35(57.44 \%) \mathrm{d} 0.00(\quad 0.08 \%)$

141. (1.67797) BD ( 1)Rh 136 - P 137

( $15.15 \%) \quad 0.3893 *$ Rh 136 s( 36.01\%)p 1.61( 57.91\%)d 0.17( $\quad 6.08 \%)$

( 84.85\%) $\quad 0.9211 *$ P 137 s( 49.58\%)p 1.02( 50.39\%)d 0.00( $0.04 \%)$

142. (1.67663) BD ( 1)Rh 136 - P 138

( $17.63 \%) \quad 0.4199 *$ Rh 136 s( $39.93 \%)$ p 1.31( 52.13\%)d 0.20( $7.95 \%)$

( 82.37\%) $\quad 0.9076 *$ P 138 s( 42.48\%)p 1.35( 57.44\%)d 0.00( $0.08 \%)$

\section{Donations}

\begin{tabular}{|c|c|c|}
\hline \multirow{5}{*}{$\mathbf{R} \mathbf{h}_{21}$} & $\mathbf{U}_{19}$ & $\begin{array}{ll}540,08 & (\text { CR 16,14\%+LP } \\
& 83,86 \%)\end{array}$ \\
\hline & $\mathbf{U}_{20}$ & $538,29 \quad(C R 14,17 \%+L P 85,83 \%)$ \\
\hline & $\mathbf{U}_{135}$ & $155,28 \quad(C R 3,35 \%+L P 96,65 \%)$ \\
\hline & $\mathbf{U}_{134}$ & 45,25(CR 3,80\%+LP 96,20\%) \\
\hline & $\mathbf{R h}_{136}$ & 18,81(CR 4,63\%+LP 95,37\%) \\
\hline
\end{tabular}




\begin{tabular}{|c|c|c|c|}
\hline \multirow{5}{*}{$\mathbf{R h}_{136}$} & $\mathrm{U}_{19}$ & \multicolumn{2}{|c|}{ 45,24(CR 3,80\%+LP 96,20\%) } \\
\hline & $\mathbf{U}_{\mathbf{2 0}}$ & \multicolumn{2}{|c|}{$129,64 \quad(C R 3,64 \%+L P$ 96,36\% } \\
\hline & $\mathbf{U}_{134}$ & 539,97 & $\begin{array}{l}\text { (CR 16,14\%+LP } \\
3,86 \%)\end{array}$ \\
\hline & $\mathbf{U}_{135}$ & 453,41 & $\begin{array}{l}\text { (CR 15,10\%+LP } \\
4,90 \%)\end{array}$ \\
\hline & $\mathbf{R h}_{21}$ & \multicolumn{2}{|c|}{ 18,03(CR 0,44\%+LP 99,56\%) } \\
\hline
\end{tabular}

\begin{tabular}{|c|c|c|}
\hline \multirow{4}{*}{$\mathrm{U}_{19}$} & $\mathbf{U}_{\mathbf{2 0}}$ & 51,2 (CR 41,70\%+LP 58,30\%) \\
\hline & $\mathbf{R h}_{21}$ & $12,41($ CR 16,60\%+LP 83,40\%) \\
\hline & $\mathbf{U}_{134}$ & 0,31 (CR 77,42\%+LP 22,58\%) \\
\hline & $\mathbf{U}_{136}$ & 0,28 (CR 64,29\%+LP 35,71\%) \\
\hline \multirow{5}{*}{$\mathbf{U}_{\mathbf{2 0}}$} & $\mathrm{U}_{19}$ & 80,58 (CR 39,50\%+LP 60,50\%) \\
\hline & $\mathbf{R h}_{21}$ & 37,47 (CR 30,42\%+LP 69,58\%) \\
\hline & $\mathbf{U}_{134}$ & 19,66 (CR 74,87\%+LP 25,13\%) \\
\hline & $\mathbf{U}_{135}$ & 62,48 (CR 47,57\%+LP 52,43\%) \\
\hline & $\mathbf{R h}_{136}$ & 2,36 (CR 61,44\%+LP 38,56\%) \\
\hline
\end{tabular}

\begin{tabular}{|c|c|c|}
\hline \multirow{4}{*}{$\mathrm{U}_{135}$} & $\mathrm{U}_{19}$ & 28,98 (CR 83,20\%+LP 16,80\%) \\
\cline { 2 - 3 } & $\mathrm{U}_{20}$ & 67,87 (CR 48,27\%+LP 51,73\%) \\
\cline { 2 - 3 } & $\mathrm{U}_{134}$ & $\mathbf{8 2 , 2 8}$ (CR 39,95\%+LP 60,05\%) \\
\cline { 2 - 3 } & $\mathrm{Rh}_{21}$ & $2,38($ CR 62,61\%+LP 37,39\%) \\
\cline { 2 - 3 } & $\mathrm{Rh}_{136}$ & 39,25 (CR 31,21\%+LP 68,79\%) \\
\hline
\end{tabular}

\begin{tabular}{|c|c|c|}
\hline \multirow{4}{*}{$U_{134}$} & $\mathrm{U}_{20}$ & 9,68 (CR 57,54\%+LP 42,46\%) \\
\cline { 2 - 3 } & $\mathrm{U}_{135}$ & 42,3 (CR 41,37\%+LP 58,63\%) \\
\cline { 2 - 3 } & $\mathrm{Rh}_{21}$ & $\mathbf{0 , 2 8}$ (CR 64,29\%+LP 35,71\%) \\
\cline { 2 - 3 } & $\mathrm{Rh}_{136}$ & 12,41 (CR 16,60\%+LP 83,40\%) \\
\hline
\end{tabular}

\begin{tabular}{|l|c|c|}
\hline \multirow{3}{*}{$N_{26}$} & $U_{19}$ & $138.16($ CR 10\% +LP 90\%) \\
\cline { 2 - 3 } & $U_{20}$ & $122,33($ CR 11\% +LP 89\%) \\
\cline { 2 - 3 } & $\mathbf{U}_{134}$ & $2.48($ LP 100\%) \\
\cline { 2 - 3 } & $\mathbf{U}_{135}$ & $116,38($ CR 12\% +LP 88\%) \\
\hline
\end{tabular}

\begin{tabular}{|l|c|c|}
\hline \multirow{3}{*}{$N_{141}$} & $U_{19}$ & $2.26($ LP 100\%) \\
\cline { 2 - 3 } & $U_{20}$ & $121.20($ CR 13\% +LP 87\%) \\
\cline { 2 - 3 } & $U_{134}$ & $138.17($ CR 10\% +LP 90\%) \\
\cline { 2 - 3 } & $U_{135}$ & $104.64($ CR 14\% +LP 86\%) \\
\hline
\end{tabular}


OM
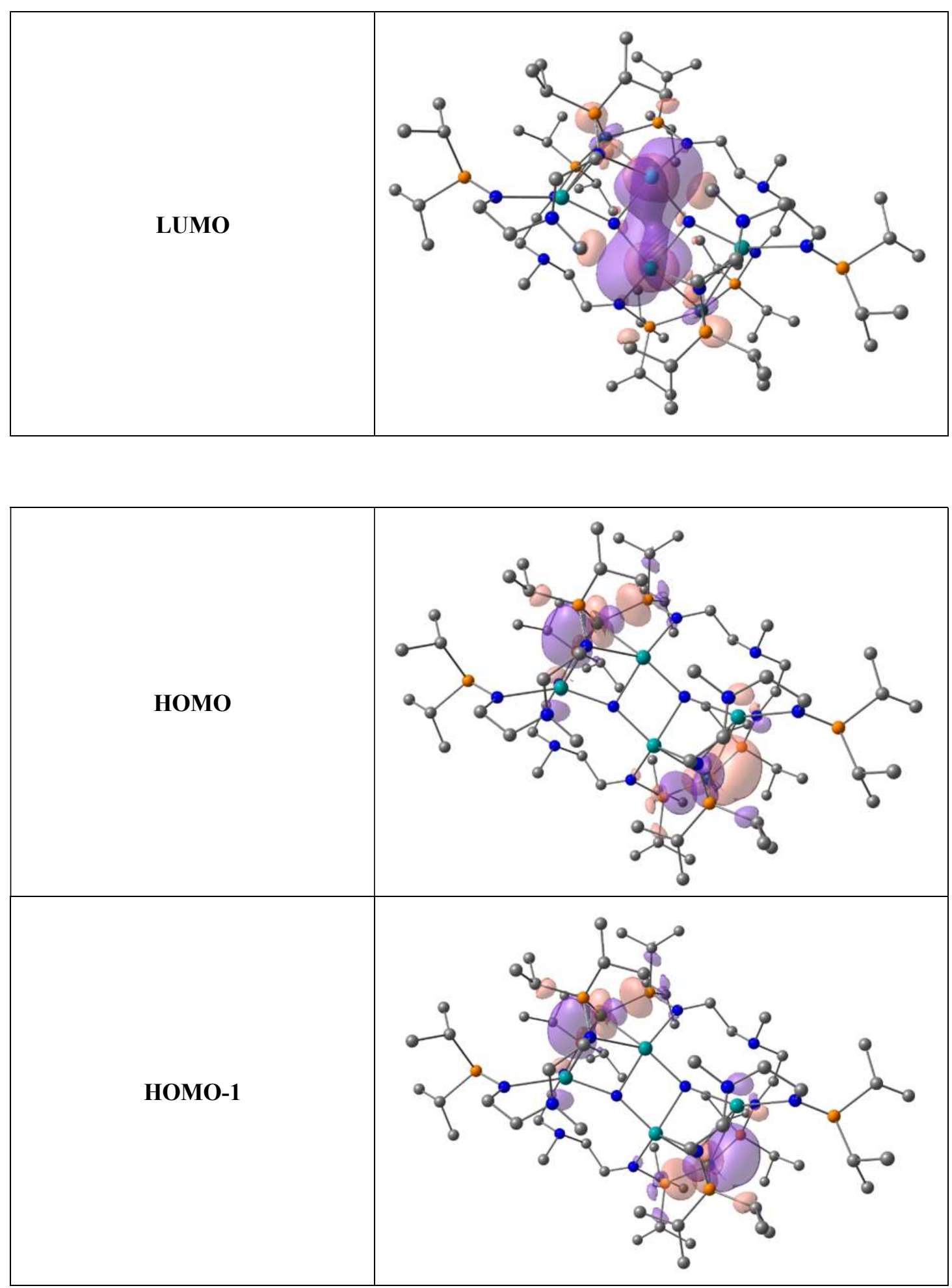


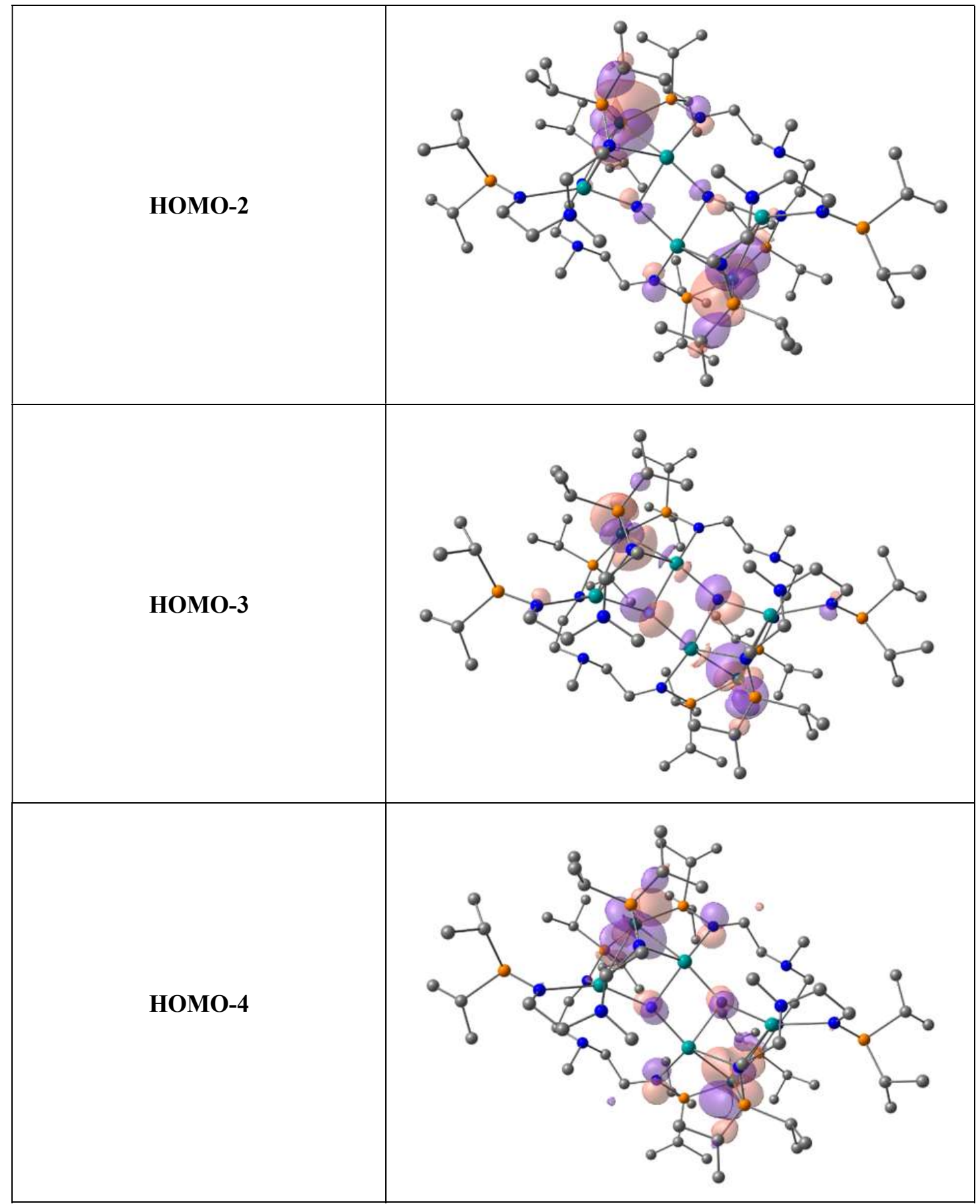




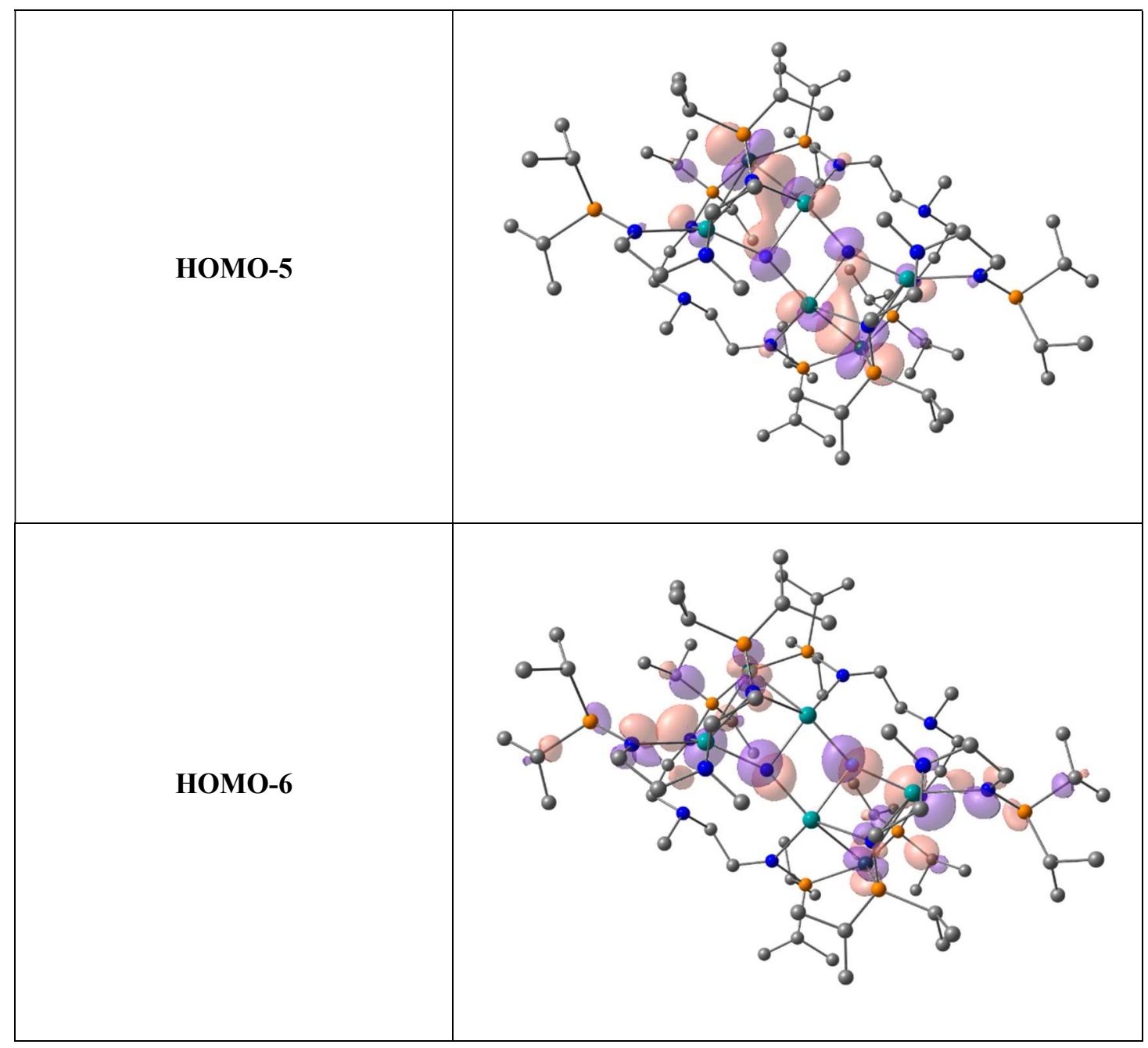




\section{Intermediate A}

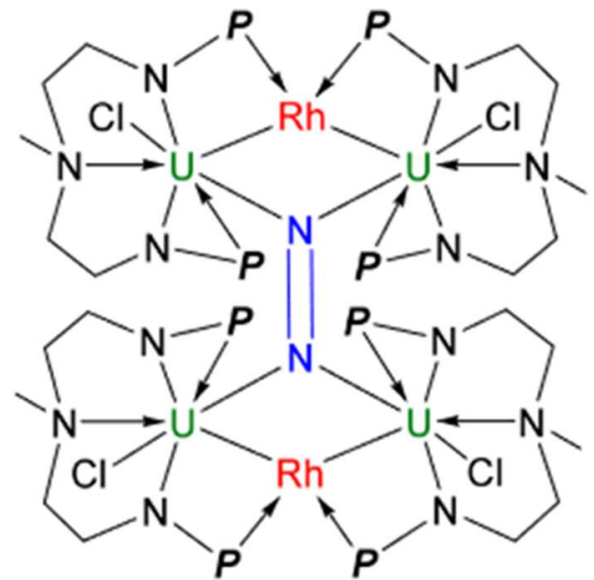

A

\begin{tabular}{|c|c|}
\hline \multicolumn{2}{|c|}{ Small Core Unpaired Spin density } \\
\hline U1 & 2.10 \\
\hline U2 & 2.08 \\
\hline U3 & 2.08 \\
\hline U4 & 2.06 \\
\hline
\end{tabular}

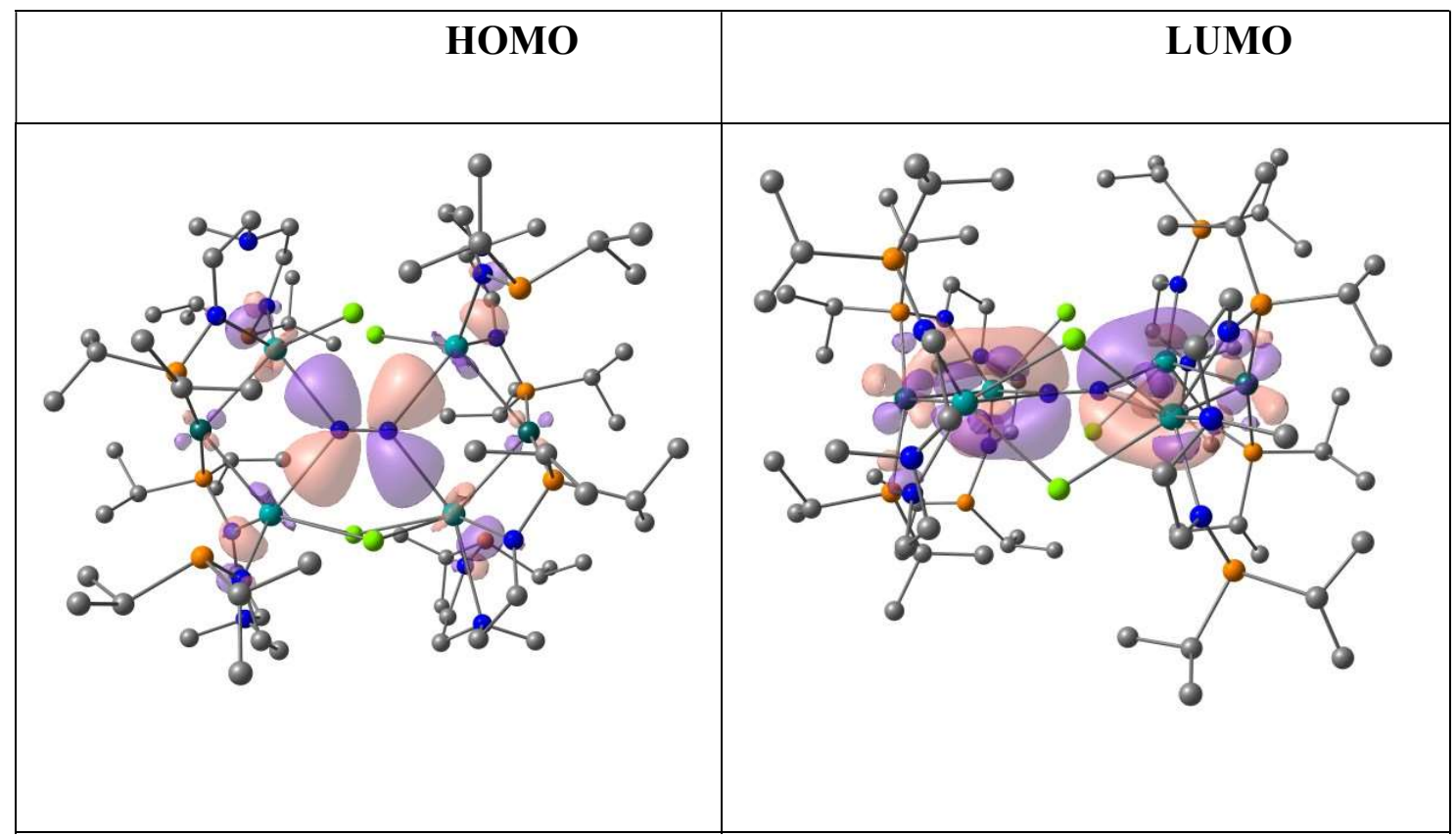




\section{$\underline{\text { Intermediate B }}$}
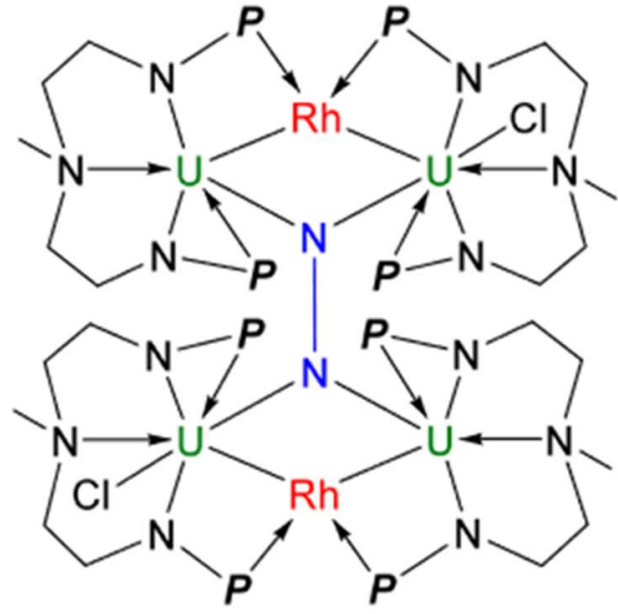

B

\begin{tabular}{|c|c|}
\hline \multicolumn{2}{|c|}{ Small Core Unpaired Spin density } \\
\hline U1 & 2.07 \\
\hline U2 & 2.06 \\
\hline U3 & 2.07 \\
\hline U4 & 2.05 \\
\hline
\end{tabular}

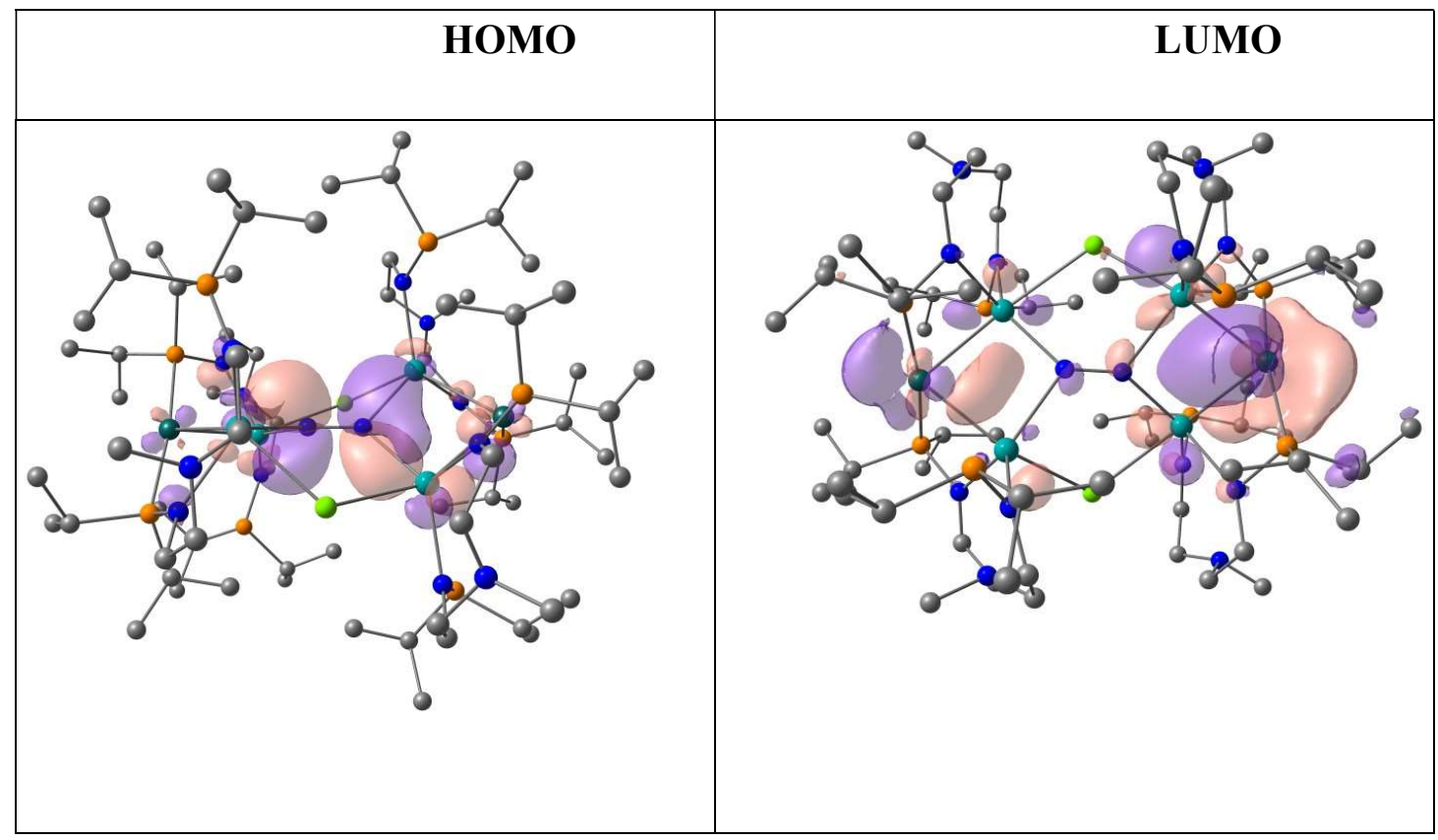


130

\section{Complex 3}

U $\quad 5.9232937 .41405615 .679993$

U $\quad 10.2777686 .43447118 .274777$

Rh 9.2594538 .20412815 .555216

Cl 4.7705755 .01445015 .560999

Cl 9.4931466 .66685920 .812508

Cl 7.3873949.800802 15.532191

Cl 7.493570 6.270760 17.744141

Cl 11.1087156 .58580415 .592638

P $\quad 4.4285298 .73127118 .023480$

P $\quad 10.2070203 .38760217 .848572$

P $\quad 8.4410607 .19879313 .570121$

P $\quad 10.3519259 .53127117 .186719$

N $\quad 4.0213318 .29476816 .399358$

$\mathrm{N} \quad 6.7193637 .16142113 .580211$

$\mathrm{N} \quad 4.1785097 .98896113 .785387$

N $\quad 11.2055564 .48031618 .747536$

$\mathrm{N} \quad 11.1404808 .51957118 .335391$

$\mathrm{N} \quad 12.6838726 .58152219 .359751$

C 3.6587286 .25297919 .081062

C $\quad 2.7523398 .48787715 .721392$ 

C $\quad 1.8449598 .01128219 .224087$
C $\quad 10.07702011 .87280718 .906641$
C 3.99634810 .96075119 .693257
C $\quad 11.02915711 .64508715 .458227$
C $\quad 10.2010631 .26444619 .871471$
C 8.3669333 .00807819 .932353
C $\quad 4.50033111 .44617517 .270830$
C $\quad 10.6730881 .12182416 .224715$
C $\quad 3.3508177 .74297219 .235467$
C $\quad 12.7246139 .79015015 .634954$
C 8.4292824 .45598514 .124954
C $\quad 9.3228122 .20709919 .046969$
C $\quad 3.79545110 .50517918 .245487$
C 8.36075810 .03519319 .086393
C $\quad 12.2744733 .05382515 .968902$
C $\quad 4.4566677 .03549312 .698164$
C $\quad 10.5000048 .17261111 .921561$
C 8.8342144 .99777911 .696519
C $\quad 12.7000967 .86459620 .082760$
C $\quad 4.3392349 .37582913 .329797$
C $\quad 9.30789510 .78478918 .150613$
C $\quad 13.7327476 .53850618 .332917$ 
C 11.4220512 .24564216 .944996

C $\quad 5.9449157 .04419012 .351171$

C $\quad 2.8407007 .78191014 .368731$

C 8.4791909 .65684812 .126563

C $\quad 12.3627574 .15410519 .561275$

C $\quad 11.67993510 .61385216 .384407$

C $\quad 9.0446745 .45300813 .144059$

C 8.9764798 .21510212 .063711

C $\quad 12.2388968 .99694219 .165412$

C $\quad 12.7986425 .43256420 .275905$

H 4.7288826 .04834319 .176332

H $\quad 3.1354295 .67776219 .854719$

H $\quad 3.3371545 .87392918 .105915$

H $\quad 1.9080108 .05906216 .275554$

H 2.5262539 .55916115 .594611

H $\quad 1.3778707 .63676918 .308096$

H $\quad 1.3677897 .48759320 .062075$

H $\quad 1.5965009 .07249819 .322190$

H $\quad 10.77674012 .43020818 .277646$

H $\quad 9.36259112 .59811919 .315271$

H $\quad 10.63239411 .46458219 .756287$

H $\quad 5.05025610 .89330119 .985870$ 
H 3.68841112 .00734619 .806341

H $\quad 3.41302010 .37005720 .405299$

H $\quad 10.33923012 .31263515 .982088$

H $\quad 11.80167112 .26713814 .989946$

H $\quad 10.46749211 .14738514 .661118$

H $\quad 10.7516821 .81004220 .643824$

H $\quad 9.5756540 .52503320 .387379$

H $\quad 10.9253080 .71281019 .264353$

H $\quad 7.6856413 .62610019 .340265$

H $\quad 7.7606782 .32829620 .543627$

H $\quad 8.9100023 .67378720 .610673$

H $\quad 4.36744211 .13422116 .231576$

H $\quad 4.10332512 .46404017 .371887$

H $\quad 5.57700511 .47994317 .462392$

H 9.9371191 .52315315 .518939

H $\quad 11.3772580 .50603615 .652152$

H $\quad 10.1466990 .45790416 .916108$

H $\quad 3.7537858 .06942920 .205455$

H $\quad 12.2766079 .25975514 .790685$

H $\quad 13.51146310 .45033415 .249379$

H $\quad 13.1955159 .03736316 .271586$

H 7.3399524 .41487414 .028216 
H 8.8200423 .45090913 .924436

H 8.6654094 .69856315 .164615

H $\quad 8.7144871 .60388018 .357041$

H $\quad 2.72082010 .50138118 .019988$

H $\quad 8.9059079 .49175619 .864192$

Н $\quad 7.69174910 .74767419 .584100$

H 7.7357769 .31599018 .550413

H $\quad 12.7986803 .87392816 .466765$

H $\quad 13.0247512 .40877315 .494879$

H $\quad 11.6589803 .49679115 .180084$

Н $\quad 4.1799896 .04446113 .066177$

H $\quad 3.8481997 .25850211 .805736$

H $\quad 10.8877047 .15748211 .798736$

H $\quad 10.8103088 .75509611 .045518$

H $\quad 10.9793448 .60722112 .804549$

H $\quad 9.2478135 .68903310 .956610$

H $\quad 9.3320494 .03156011 .548122$

H $\quad 7.7756624 .84551711 .466499$

H $\quad 11.9992897 .76874220 .915818$

H $\quad 13.7018298 .08003220 .490941$

H $\quad 5.3569409 .53721712 .973583$

H $\quad 3.6296549 .61164712 .520872$ 
H 4.16879810 .06225814 .160791

H 8.70969511 .26039617 .364959

H $\quad 13.5788337 .33718117 .606922$

H $\quad 14.7324266 .64456318 .783474$

H $\quad 13.6885685 .59061617 .794347$

H $\quad 12.0793021 .80216417 .704985$

H $\quad 6.1682147 .87210611 .660017$

H 6.1578356 .11943011 .798165

H $\quad 2.0488818 .12607513 .682979$

H 2.7227646 .70629014 .523690

H $\quad 8.91399110 .18427512 .979324$

H $\quad 8.76445810 .18778711 .209817$

H $\quad 7.3933689 .72055012 .229191$

H 12.1430293 .39622720 .323419

H $\quad 13.1810113 .74437818 .946881$

H $\quad 12.17297711 .13864417 .213073$

H $\quad 10.1215685 .50995513 .339655$

Н $\quad 8.5237547 .72212311 .193742$

H $\quad 13.0798849 .34889918 .547682$

H $\quad 11.9551319 .84271819 .806081$

H $\quad 13.8221215 .34107220 .675497$

H $\quad 12.1143855 .61904721 .107639$ 
130

smallcore-Complex 3

U $\quad 5.9962897 .36577915 .698965$

U $\quad 10.1830356 .48981118 .259377$

Rh 9.2068878 .15235615 .606829

Cl 4.7365655 .02978615 .542688

Cl 9.4692996 .67831120 .812553

Cl 7.388614 9.765125 15.464491

Cl 7.4263906 .17366517 .799367

Cl 11.116755 6.643465 15.633741

P $\quad 4.6286558 .72549717 .999373$

P $\quad 10.1587153 .54179117 .710910$

P $\quad 8.4709537 .05183813 .676130$

P $\quad 10.1833139 .52873917 .222745$

$\mathrm{N} \quad 4.1450318 .31529816 .400792$

N $\quad 6.7660777 .07214113 .610042$

N $\quad 4.3022428 .01293813 .812130$

$\mathrm{N} \quad 11.1505574 .56189618 .675929$

N $\quad 11.0234938 .57016318 .356707$

N $\quad 12.5795916 .65287419 .297498$

C 3.8011656 .28169919 .001380

C 2.8951818 .59130915 .729436 
C 2.0532848 .09904419 .183979

C $\quad 9.76609911 .70816919 .046307$

C $\quad 4.23711710 .94816519 .673521$

C $\quad 10.69561811 .69831115 .552403$

C $\quad 10.1802941 .33041719 .584784$

C 8.4197933 .12985019 .827987

C $\quad 4.87253811 .40507317 .272864$

C $\quad 10.6260391 .35123016 .015064$

C $\quad 3.5442537 .77280019 .208781$

C $\quad 12.4479889 .88796315 .615716$

C $\quad 8.3576944 .41630614 .483519$

C $\quad 9.2819792 .32709018 .856204$

C $\quad 4.07786010 .51153018 .218485$

C 8.1303249 .79291819 .038451

C $\quad 12.1972953 .31365415 .815483$

C $\quad 4.5303897 .06139712 .714658$

C $\quad 10.6265167 .80036412 .081925$

C $\quad 8.7338964 .73256712 .012808$

C $\quad 12.6203877 .92123820 .039264$

C $\quad 4.5471589 .39090613 .375755$

C $\quad 9.05145010 .66348018 .192953$

C $\quad 13.5725296 .63628918 .220034$ 
C $\quad 11.3693502 .45534316 .764467$

C $\quad 6.0155727 .01217012 .370075$

C $\quad 2.9523877 .87680014 .381667$

C $\quad 8.7164369 .43606912 .230592$

C $\quad 12.3200794 .22312519 .453973$

C $\quad 11.42629910 .67870816 .424540$

C $\quad 9.0140075 .27661913 .411386$

C $\quad 9.1102647 .96507012 .170687$

C $\quad 12.1393659 .05911019 .144273$

C $\quad 12.7443285 .49314420 .187235$

H 4.8626596 .03925919 .097482

H $\quad 3.2475985 .69314519 .742263$

H 3.4814365 .95926918 .005654

H $\quad 2.0250788 .22005416 .283266$

H $\quad 2.7433209 .67452115 .599121$

H $\quad 1.5896577 .73874618 .261344$

H $\quad 1.5451757 .59675420 .015953$

H $\quad 1.8471819 .16983519 .272119$

H 10.47100912 .32223518 .478586

H $\quad 9.02657212 .38586619 .488698$

H $\quad 10.30593211 .24106319 .874612$

H $\quad 5.27132710 .82298020 .010779$ 
H $\quad 3.97967612 .00817719 .781847$

H $\quad 3.59415310 .38073520 .351618$

H $\quad 10.02752212 .34339816 .129317$

H $\quad 11.41932412 .34028215 .037329$

H $\quad 10.09153211 .19050414 .794305$

H $\quad 10.8006911 .83759620 .328797$

H $\quad 9.5690610 .59504020 .121653$

H $\quad 10.8429720 .77875118 .911546$

H $\quad 7.7273843 .79194919 .301364$

H $\quad 7.8323732 .45527020 .461594$

H $\quad 9.0370943 .75523520 .480257$

H 4.80040811 .06300316 .237636

H $\quad 4.50704012 .43772117 .322443$

Н $\quad 5.93439811 .40302917 .534128$

H 9.8985541 .77383615 .314551

H $\quad 11.3316940 .74589715 .434399$

H $\quad 10.0889120 .67789516 .688326$

H $\quad 3.9570738 .06218020 .185398$

H 11.9648829 .39015214 .770931

H $\quad 13.22349310 .55870315 .228073$

H $\quad 12.9294329 .10795016 .209551$

H $\quad 7.2666044 .44382114 .405795$ 
H $\quad 8.6702583 .37266714 .368127$

H $\quad 8.6363184 .72993415 .491986$

Н $\quad 8.6171541 .77894218 .173922$

H $\quad 3.01547210 .55668117 .945909$

H $\quad 8.6928419 .20881119 .773049$

H 7.42132610 .42175819 .587780

H 7.5467539 .09911318 .429173

H $\quad 12.7042024 .12583816 .341780$

H $\quad 12.9564882 .70387015 .311282$

H $\quad 11.5635193 .77453115 .052819$

H 4.2154276 .08031413 .076648

H $\quad 3.9285597 .32308111 .829361$

H 10.9282946 .75852911 .945709

H $\quad 11.0189448 .37783711 .237142$

H 11.1031198 .16535012 .996754

H 9.1611845 .34884711 .217050

H $\quad 9.1655473 .72937011 .916976$

H $\quad 7.6595404 .63639211 .834587$

H $\quad 11.9354277 .81530320 .883565$

H $\quad 13.6323938 .12488220 .425261$

H $\quad 5.5741619 .49327913 .026989$

H $\quad 3.8555949 .67711812 .568213$ 
H 4.41559910 .07307914 .216152

H 8.45561311 .16552817 .423533

H $\quad 13.3726937 .44892317 .522413$

H $\quad 14.5934026 .73583118 .620004$

H $\quad 13.4962495 .70157517 .664113$

H $\quad 12.0345241 .99722117 .507969$

H $\quad 6.2821607 .84750911 .703874$

H 6.1985846 .09283311 .799231

H 2.1869918 .25862613 .687099

H $\quad 2.7817206 .80945914 .541787$

H 9.2130079 .92806913 .070940

H $\quad 9.0108759 .94423911 .305038$

H 7.6419269 .57004512 .373255

H 12.1218073 .44386520 .198919

H $\quad 13.1308763 .84248718 .812547$

H $\quad 11.93565211 .19693717 .246629$

H $\quad 10.0943895 .30049813 .586280$

H $\quad 8.6388497 .49755911 .296968$

H 12.9588449 .40836218 .497281

H $\quad 11.8738029 .90620419 .789484$

H $\quad 13.7784825 .42015620 .560179$

H $\quad 12.0728595 .64851721 .035132$ 
128

\section{Complex 4}

U $\quad 8.9071241 .96136513 .251048$

U $\quad 7.9165391 .93192717 .610934$

Rh 9.3262363 .27224715 .655347

Cl 7.4369600 .46825115 .197385

Cl $9.122637-0.46933512 .174858$

Cl 5.4309791 .10367918 .108522

P $\quad 9.802751-0.47326018 .513347$

P $\quad 6.1414793 .11885712 .140067$

P $\quad 11.4402042 .37746815 .110284$

P $\quad 7.6555834 .80380016 .301342$

N $\quad 8.7507840 .65822719 .280695$

N 7.6847892 .68644011 .496531

$\mathrm{N} \quad 10.2908202 .57982611 .003877$

$\mathrm{N} \quad 11.1616302 .14753013 .438645$

N $\quad 7.6098772 .89430120 .121973$

N $\quad 7.2934704 .10937517 .820915$

C $\quad 12.316605-1.61805319 .129458$

C $\quad 7.674967-2.22849718 .014497$

C $\quad 9.037879-2.88903120 .037497$

C $\quad 9.062097-2.22607318 .658387$ 

C $\quad 4.2549945 .16204511 .580060$
C $\quad 4.5040541 .88286110 .012350$
C $\quad 5.1779750 .49256112 .010602$
C $\quad 4.8238301 .89331711 .508930$
C $\quad 13.0333043 .92160416 .819558$
C $\quad 7.3635721 .71712420 .966599$
C 8.4295490 .65269220 .699255
C $\quad 11.9707870 .77031619 .862316$
C $\quad 11.313670-0.59386319 .665266$
C $\quad 9.4283902 .1645389 .889010$
C 8.0219962 .73298410 .081653
C $\quad 6.6854165 .82019711 .458877$
C $\quad 5.6683454 .70935711 .207071$
C $\quad 13.7577590 .65877615 .536752$
C $\quad 10.5826684 .01333610 .937465$
C $\quad 11.5305541 .79354811 .074760$
C $\quad 12.2016431 .99644312 .437241$
C $11.498621-0.41221915 .176010$
C $\quad 12.2480370 .78452215 .759094$
C $\quad 12.4452564 .97202414 .603131$
C $\quad 12.8005903 .67683915 .327144$
C $\quad 5.0914093 .93830415 .612555$ 
C 5.2876756 .44414415 .839212

C $\quad 6.0023325 .15149215 .434596$

C $\quad 8.7131417 .16309615 .217700$

C 8.8101473 .61292120 .554490

C $\quad 6.4495113 .79475020 .065552$

C $\quad 6.6140154 .78763218 .909660$

C 8.3707516 .53546716 .571148

C $\quad 9.5918926 .49340117 .484872$

H $\quad 7.696256-1.84047216 .992153$

H $\quad 7.277050-3.24995317 .975951$

H $\quad 6.967669-1.61605118 .583151$

H $\quad 8.028782-0.31225221 .035847$

H $\quad 9.3253860 .84091521 .314132$

H $\quad 8.336167-2.39045120 .712866$

H $\quad 8.702633-3.92967819 .941870$

H $\quad 10.017620-2.90967020 .524210$

H $\quad 14.3339241 .44594616 .031441$

H $\quad 14.107872-0.29930615 .940387$

H $\quad 14.0182910 .66951514 .473703$

H $\quad 12.680587-1.33260718 .136247$

H $\quad 13.187781-1.68490219 .792736$

Н $\quad 11.888650-2.62158419 .049721$ 
H $\quad 13.3465943 .01720517 .349879$

H $\quad 13.8136764 .67799416 .967241$

H $\quad 12.1165254 .28442517 .297459$

H $\quad 5.3349651 .4755459 .428360$

H $\quad 3.6361031 .2386389 .822613$

H 4.2640162 .8734029 .613876

H $\quad 5.3129580 .46647613 .095717$

H $\quad 4.379708-0.21509011 .755331$

H $\quad 6.1044610 .12717611 .554992$

H $\quad 11.2653661 .51176020 .245821$

H $\quad 12.8020810 .69635220 .574179$

H $\quad 12.3762101 .15996818 .922700$

H $\quad 4.1729005 .36299812 .654123$

H 3.9992526 .08903211 .052262

H 3.4958934 .41811011 .322158

H $\quad 9.733612-2.80782718 .009613$

H 11.4862735 .36485814 .957433

H $\quad 13.2150465 .73278014 .782928$

H 12.3646964 .82747113 .522805

H $\quad 4.7911683 .80502416 .655230$

H 4.1825024 .06237915 .011785

H $\quad 5.5706613 .01336215 .279845$ 
H $\quad 3.9278102 .22927112 .051826$

H $\quad 10.943625-0.94029620 .638751$

H $11.650731-0.50093214 .097014$

H $\quad 11.851663-1.33882515 .644144$

H $\quad 10.422352-0.34975215 .359499$

H 7.7066725 .49763911 .239816

H $\quad 6.4646046 .69321810 .832496$

H 6.6633796 .15134412 .502420

H $\quad 5.5718753 .17112119 .880448$

H $\quad 6.3048744 .32612621 .021567$

Н $\quad 7.8379157 .27131414 .570067$

H $\quad 9.1457858 .16122215 .357462$

H 9.4462856 .55034014 .681406

H $\quad 5.8715607 .34498115 .630382$

H $\quad 4.3480506 .53189615 .280114$

H $\quad 5.0270496 .45225816 .902163$

H $\quad 11.2485040 .74365910 .967734$

H $\quad 12.2207432 .05478710 .254585$

H $\quad 9.0132104 .43747219 .869041$

H $\quad 8.6934434 .01469021 .574291$

H $\quad 9.6708592 .94230420 .537960$

H $\quad 12.0469360 .81163116 .836831$ 
H $\quad 11.1392834 .32022411 .824374$

H $\quad 11.1727534 .26221810 .040243$

H 9.6517314 .58208310 .910886

H $\quad 5.6858984 .46127910 .137893$

H $\quad 7.1781985 .67574519 .240076$

H $\quad 5.6123765 .14738118 .633006$

Н $\quad 7.3354981 .98677122 .036308$

H $\quad 6.3846461 .32207120 .684171$

H $\quad 10.3747965 .85888517 .056594$

H $\quad 10.0033377 .50174717 .617617$

H 9.3491186 .09780718 .474492

H $\quad 7.3346332 .1401709 .464977$

H 7.9675333 .7630819 .692027

H $\quad 13.7126683 .25929114 .883260$

H $\quad 6.2783145 .20960914 .374659$

H $\quad 7.5843867 .12849617 .054568$

H $\quad 12.8646062 .87723712 .415562$

H $\quad 12.8518821 .12942712 .623228$

H $\quad 9.8476922 .4751098 .916617$

H 9.3808561 .0732119 .912398 
128

smallcore-Complex 4

U 8.9004011 .91274513 .309444

U $\quad 7.8745871 .92104717 .538685$

Rh 9.2989163 .25375015 .646390

Cl 7.371156 0.433637 15.180199

Cl 9.112671 -0.509919 12.229346

Cl 5.4080401 .08422218 .080885

P $\quad 9.810120-0.31681018 .357892$

P $\quad 6.2828593 .14427712 .308108$

P $\quad 11.3784712 .31556915 .167377$

P $\quad 7.5997864 .74134816 .224808$

N $\quad 8.7286420 .70033419 .205245$

$\mathrm{N} \quad 7.7304382 .63804711 .552385$

$\mathrm{N} \quad 10.2894382 .47280211 .088723$

$\mathrm{N} \quad 11.1439862 .08029613 .501214$

N $\quad 7.5570182 .88065120 .010097$

$\mathrm{N} \quad 7.2179264 .07528717 .740104$

C $\quad 12.379886-1.35191318 .905782$

C $7.736722-2.08885317 .896676$

C $\quad 9.231619-2.78144519 .809457$

C $\quad 9.160390-2.09094218 .449459$ 

C 4.5099685 .30910911 .924080
C $\quad 4.4547892 .10827910 .289117$
C $\quad 5.2800390 .57603512 .119810$
C 4.8819662 .00364611 .751148
C $\quad 12.8871183 .85224616 .911143$
C $\quad 7.3508701 .72172020 .886709$
C 8.4358530 .67691120 .621256
C $\quad 11.9111751 .00899719 .677621$
C $\quad 11.338495-0.38727519 .467910$
C $\quad 9.4412242 .0754169 .959382$
C $\quad 8.0383582 .65942710 .139174$
C $\quad 6.9752925 .78240611 .636952$
C $\quad 5.8550154 .76767711 .444777$
C $\quad 13.6329100 .58678015 .631771$
C $\quad 10.5779653 .90480711 .056834$
C $\quad 11.5270881 .68851511 .160407$
C $\quad 12.1925441 .89946012 .523165$
C $\quad 11.355673-0.43998615 .222993$
C $\quad 12.1262930 .72745215 .829814$
C $\quad 12.3077234 .88919314 .684023$
C $\quad 12.7101773 .61356415 .413330$
C $\quad 5.1025823 .80239315 .536487$ 
C $\quad 5.2443896 .32060715 .726029$

C $\quad 5.9739445 .03733715 .337758$

C 8.6894477 .03144615 .112693

C $\quad 8.7649283 .61794720 .373294$

C $\quad 6.3882393 .76663819 .975472$

C $\quad 6.5207824 .75158618 .809886$

C 8.3182006 .45484116 .477367

C $\quad 9.5279156 .36528417 .399355$

H $\quad 7.692071-1.67102116 .887794$

H $\quad 7.341716-3.11043717 .856064$

H $\quad 7.067717-1.49323618 .526255$

H $\quad 8.060032-0.29680520 .959374$

H $\quad 9.3366490 .88582321 .221615$

H $\quad 8.583659-2.28740920 .538694$

H $\quad 8.884493-3.81794619 .720275$

H $\quad 10.242956-2.81218120 .224447$

H $\quad 14.2028101 .35572916 .160474$

H $\quad 13.968960-0.38569216 .010854$

H 13.9093090 .63085114 .574446

H $\quad 12.737582-1.02070117 .926826$

H $\quad 13.248589-1.41043519 .571876$

Н $\quad 11.983908-2.36425318 .788122$ 
H $\quad 13.2528692 .96245517 .431110$

H $\quad 13.6028864 .66207117 .093402$

H $\quad 11.9314964 .13306717 .368228$

H $\quad 5.2628321 .8028099 .618785$

H 3.6068211 .43946910 .098638

H $\quad 4.1437243 .11728810 .003738$

H $\quad 5.4996800 .47772813 .185883$

H $\quad 4.470571-0.12148211 .876048$

H $\quad 6.1741040 .25972111 .572409$

H $\quad 11.1573171 .69597820 .068039$

H $\quad 12.7483160 .98284220 .384817$

H $\quad 12.2818051 .43449718 .740880$

H 4.5343865 .54544412 .991785

H 4.2568116 .23179711 .389028

H 3.6958264 .59799511 .760123

H $\quad 9.804658-2.63187117 .741698$

H $\quad 11.3247005 .22942315 .028583$

H $\quad 13.0360655 .68763614 .867924$

H $\quad 12.2396664 .73049413 .605337$

H $\quad 4.7968963 .68688016 .578919$

H 4.1991623 .87562214 .919831

H $\quad 5.6235402 .88845315 .238224$ 
H $\quad 4.0398292 .30405112 .391077$

H $\quad 10.991912-0.76734620 .437024$

H $\quad 11.529900-0.52591414 .147930$

H $\quad 11.663075-1.37975415 .696308$

H $\quad 10.278138-0.33815615 .380217$

H 7.9256375 .40589811 .253452

H $\quad 6.7446846 .71859711 .115387$

H 7.1245946 .01807612 .694580

H $\quad 5.5164153 .13100019 .809788$

H $\quad 6.2605074 .29993220 .931807$

H $\quad 7.8129277 .18430514 .476714$

H $\quad 9.1926557 .99874715 .224135$

H $\quad 9.3682436 .35335214 .583241$

H $\quad 5.8134267 .22175815 .481682$

H $\quad 4.2897406 .38229015 .190282$

H $\quad 5.0141256 .34771916 .794948$

H $\quad 11.2420100 .63959111 .060408$

H $\quad 12.2153541 .94795210 .339181$

H $\quad 8.9370014 .41045719 .644261$

H $\quad 8.6833634 .05847521 .379540$

H $\quad 9.6245962 .94802020 .347773$

H $\quad 11.8978510 .75885516 .899263$ 
H $\quad 11.1201044 .18851611 .959447$

H 11.1773594 .17596910 .173269

H $\quad 9.6442704 .46697911 .035535$

H $\quad 5.7784804 .53193310 .376080$

H $\quad 7.0611435 .65962719 .124817$

H $\quad 5.5101615 .07569818 .525060$

H 7.3327872 .01767321 .948596

H $\quad 6.3786391 .29934120 .623653$

H $\quad 10.2577475 .65207517 .000683$

H $\quad 10.0148947 .34268817 .496424$

H $\quad 9.2479616 .02429718 .398714$

H 7.3416162 .0556339 .544493

H 7.9860033 .6826119 .732198

H $\quad 13.6429553 .22619714 .987011$

H 6.2598055 .07962614 .282208

H $\quad 7.5461747 .07798416 .944082$

H $\quad 12.8679502 .77059312 .499377$

H $\quad 12.8239331 .02439712 .730648$

H $\quad 9.8804162 .3857458 .996908$

H $\quad 9.3797710 .9853759 .981497$ 
Complex-Inter-A

\begin{tabular}{|c|c|c|c|}
\hline $\mathrm{U}$ & 5.760520 & -6.901674 & 11.378300 \\
\hline $\mathrm{U}$ & 5.390388 & -8.253312 & 7.356892 \\
\hline $\mathrm{U}$ & 2.392339 & -11.489463 & 8.815248 \\
\hline $\mathrm{U}$ & 2.018663 & -9.395800 & 12.521555 \\
\hline $\mathrm{Rh}$ & 6.940889 & -6.421249 & 8.864415 \\
\hline $\mathrm{Rh}$ & 0.839401 & -11.579573 & 11.182178 \\
\hline $\mathrm{Cl}$ & 5.215513 & -11.050731 & 7.956580 \\
\hline $\mathrm{Cl}$ & 2.956502 & -6.646732 & 11.905685 \\
\hline $\mathrm{Cl}$ & 2.580022 & -9.186639 & 7.125164 \\
\hline $\mathrm{Cl}$ & 4.823227 & -9.180227 & 13.054469 \\
\hline 1 & 8.489154 & -8.123571 & 8.274664 \\
\hline $\mathrm{P}$ & 5.743687 & -4.416658 & 9.307146 \\
\hline $\mathrm{P}$ & 8.880861 & -8.041071 & 12.803662 \\
\hline $\mathrm{P}$ & 3.919918 & -5.669309 & 5.324055 \\
\hline $\mathrm{P}$ & 3.829787 & -14.757536 & 9.342211 \\
\hline $\mathrm{P}$ & -0.705473 & -10.875598 & 9.521951 \\
\hline $\mathrm{P}$ & 2.031281 & -12.591616 & 12.964202 \\
\hline $\mathrm{P}$ & -1.059408 & -7.532325 & 12.616364 \\
\hline $\mathrm{N}$ & 6.243634 & -9.259549 & 4.883376 \\
\hline $\mathrm{N}$ & 7.602588 & -8.972571 & 7.103259 \\
\hline $\mathrm{N}$ & 5.364580 & -4.630763 & 10.946714 \\
\hline $\mathrm{N}$ & 5.897905 & -5.040450 & 13.478565 \\
\hline $\mathrm{N}$ & 7.372043 & -7.260892 & 13.118990 \\
\hline $\mathrm{N}$ & 4.449433 & -7.313625 & 5.359974 \\
\hline $\mathrm{N}$ & 4.349903 & -8.635655 & 9.844407 \\
\hline $\mathrm{N}$ & 3.448927 & -9.413756 & 10.179771 \\
\hline $\mathrm{N}$ & 3.320544 & -13.600387 & 8.163160 \\
\hline $\mathrm{N}$ & 1.527537 & -12.644201 & $\begin{array}{c}6.395611 \\
\text { s82 }\end{array}$ \\
\hline
\end{tabular}




\begin{tabular}{|c|c|c|c|}
\hline $\mathrm{N}$ & 0.180075 & -11.176939 & 8.106380 \\
\hline$N$ & 2.407806 & -11.237934 & 13.914926 \\
\hline $\mathrm{N}$ & 1.895830 & -9.093308 & 15.315871 \\
\hline $\mathrm{N}$ & 0.428602 & -7.854147 & 13.434622 \\
\hline 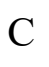 & 6.612004 & -14.250424 & 9.109895 \\
\hline $\mathrm{C}$ & 1.383953 & -16.126658 & 9.417353 \\
\hline$C$ & 3.407868 & -17.569086 & 9.684386 \\
\hline $\mathrm{C}$ & 2.828280 & -16.346912 & 8.966807 \\
\hline $\mathrm{C}$ & -1.466417 & -4.785829 & 13.708835 \\
\hline $\mathrm{C}$ & 3.900092 & -14.493854 & 14.106898 \\
\hline $\mathrm{C}$ & -0.488943 & -12.934149 & 14.186092 \\
\hline $\mathrm{C}$ & 0.544640 & -14.996976 & 13.193120 \\
\hline $\mathrm{C}$ & 0.814510 & -13.684435 & 13.930133 \\
\hline $\mathrm{C}$ & 9.455484 & -6.254304 & 6.397690 \\
\hline $\mathrm{C}$ & 8.303614 & -10.391075 & 9.887338 \\
\hline $\mathrm{C}$ & 1.145737 & -6.230360 & 5.542292 \\
\hline $\mathrm{C}$ & 6.349290 & -4.652795 & 4.346820 \\
\hline $\mathrm{C}$ & 4.302771 & -3.476267 & 3.528864 \\
\hline $\mathrm{C}$ & 4.898128 & -4.837144 & 3.902089 \\
\hline $\mathrm{C}$ & 8.259356 & -3.270096 & 9.880740 \\
\hline $\mathrm{C}$ & 3.870793 & -2.290990 & 8.678449 \\
\hline $\mathrm{C}$ & -0.497410 & -8.156893 & 8.938384 \\
\hline $\mathrm{C}$ & -1.678466 & -13.515906 & 9.644516 \\
\hline $\mathrm{C}$ & -3.099836 & -11.763235 & 10.749752 \\
\hline $\mathrm{C}$ & -2.176115 & -12.075173 & 9.572120 \\
\hline $\mathrm{C}$ & 7.027524 & -8.384123 & 4.014223 \\
\hline $\mathrm{C}$ & 10.551603 & -10.096794 & 8.781688 \\
\hline $\mathrm{C}$ & 9.340514 & -9.385570 & 9.396334 \\
\hline$C$ & 4.145657 & -8.185698 & 4.228972 \\
\hline $\mathrm{C}$ & 4.928234 & -9.509671 & $\begin{array}{c}4.304961 \\
583\end{array}$ \\
\hline
\end{tabular}




\begin{tabular}{|c|c|c|c|}
\hline $\mathrm{C}$ & 7.035342 & -10.436675 & 5.235112 \\
\hline $\mathrm{C}$ & 8.123647 & -10.020716 & 6.246063 \\
\hline $\mathrm{C}$ & 3.017836 & -4.656978 & 8.773491 \\
\hline $\mathrm{C}$ & 4.205381 & -3.765381 & 8.425475 \\
\hline $\mathrm{C}$ & 10.860238 & -6.592921 & 8.453707 \\
\hline$C$ & 9.955317 & -7.267486 & 7.422767 \\
\hline$C$ & 7.220006 & -2.604187 & 7.693293 \\
\hline $\mathrm{C}$ & 6.953412 & -2.959237 & 9.156489 \\
\hline $\mathrm{C}$ & 4.789723 & -3.627040 & 11.822409 \\
\hline $\mathrm{C}$ & 4.736879 & -4.180679 & 13.261178 \\
\hline $\mathrm{C}$ & 9.288940 & -9.092242 & 15.564895 \\
\hline $\mathrm{C}$ & 11.598465 & -7.468566 & 13.517446 \\
\hline $\mathrm{C}$ & 10.317578 & -5.680619 & 12.331515 \\
\hline $\mathrm{C}$ & 10.228595 & -6.800811 & 13.368578 \\
\hline $\mathrm{C}$ & 8.163097 & -10.572076 & 13.864529 \\
\hline $\mathrm{C}$ & 9.171935 & -9.443730 & 14.077020 \\
\hline $\mathrm{C}$ & 7.100175 & -4.219644 & 13.602982 \\
\hline $\mathrm{C}$ & 5.810546 & -5.992945 & 14.578923 \\
\hline $\mathrm{C}$ & 6.998121 & -6.971643 & 14.501292 \\
\hline $\mathrm{C}$ & 1.899472 & -5.987285 & 3.150951 \\
\hline $\mathrm{C}$ & 2.148647 & -5.561881 & 4.602596 \\
\hline $\mathrm{C}$ & 5.845064 & -16.139292 & 7.629235 \\
\hline $\mathrm{C}$ & 5.596623 & -15.379478 & 8.937477 \\
\hline $\mathrm{C}$ & 3.627799 & -13.843597 & 6.756322 \\
\hline $\mathrm{C}$ & 2.848897 & -12.891922 & 5.830918 \\
\hline $\mathrm{C}$ & 0.747782 & -13.879181 & 6.445460 \\
\hline $\mathrm{C}$ & 0.736178 & -11.590579 & 5.764080 \\
\hline $\mathrm{C}$ & -0.345562 & -11.116068 & 6.755364 \\
\hline $\mathrm{C}$ & -2.754226 & -9.147941 & 8.410261 \\
\hline $\mathrm{C}$ & -1.543040 & -9.189299 & $\begin{array}{c}9.348191 \\
584\end{array}$ \\
\hline
\end{tabular}




\begin{tabular}{|c|c|c|c|}
\hline $\mathrm{C}$ & 4.757863 & -12.825114 & 12.427185 \\
\hline $\mathrm{C}$ & 3.569165 & -13.682868 & 12.849403 \\
\hline $\mathrm{C}$ & 2.995606 & -11.271577 & 15.241056 \\
\hline $\mathrm{C}$ & 3.056088 & -9.836051 & 15.802513 \\
\hline $\mathrm{C}$ & -2.540062 & -9.459256 & 14.025503 \\
\hline $\mathrm{C}$ & 0.694008 & -9.551087 & 16.009864 \\
\hline $\mathrm{C}$ & 1.987205 & -7.638669 & 15.355207 \\
\hline $\mathrm{C}$ & 0.797807 & -7.034684 & 14.585788 \\
\hline $\mathrm{C}$ & -0.309460 & -5.034778 & 11.484466 \\
\hline $\mathrm{C}$ & -1.331030 & -5.642972 & 12.444870 \\
\hline $\mathrm{C}$ & -2.441495 & -7.940545 & 13.879678 \\
\hline $\mathrm{C}$ & -3.797042 & -7.379996 & 13.439374 \\
\hline $\mathrm{H}$ & 3.364196 & -11.927307 & 5.763989 \\
\hline $\mathrm{H}$ & 2.800147 & -13.313115 & 4.810127 \\
\hline $\mathrm{H}$ & -0.628071 & -10.094913 & 6.460265 \\
\hline $\mathrm{H}$ & -1.253495 & -11.732309 & 6.647639 \\
\hline $\mathrm{H}$ & 1.297108 & -13.904158 & 14.891320 \\
\hline $\mathrm{H}$ & 3.352562 & -14.368013 & 12.020846 \\
\hline $\mathrm{H}$ & -2.727217 & -11.933848 & 8.633319 \\
\hline $\mathrm{H}$ & 3.406796 & -14.883564 & 6.468614 \\
\hline $\mathrm{H}$ & 4.694973 & -13.685087 & 6.541186 \\
\hline $\mathrm{H}$ & -0.320940 & -11.977363 & 14.684104 \\
\hline $\mathrm{H}$ & -1.164012 & -13.532226 & 14.811072 \\
\hline $\mathrm{H}$ & -1.004492 & -12.723356 & 13.241645 \\
\hline $\mathrm{H}$ & 2.930837 & -7.353484 & 14.876520 \\
\hline $\mathrm{H}$ & 2.008923 & -7.244590 & 16.387594 \\
\hline $\mathrm{H}$ & 4.023336 & -11.663738 & 15.234973 \\
\hline $\mathrm{H}$ & 2.420342 & -11.920681 & 15.921786 \\
\hline $\mathrm{H}$ & 2.845008 & -16.526639 & 7.882940 \\
\hline $\mathrm{H}$ & 1.269367 & -14.632251 & $\begin{array}{c}7.032518 \\
\text { s85 }\end{array}$ \\
\hline
\end{tabular}




\begin{tabular}{|c|c|c|c|}
\hline $\mathrm{H}$ & 0.573033 & -14.268475 & 5.427819 \\
\hline $\mathrm{H}$ & -0.219804 & -13.686746 & 6.909812 \\
\hline & -1.866567 & -8.939468 & 10.366093 \\
\hline $\mathrm{H}$ & -0.191010 & -9.104310 & 15.561937 \\
\hline $\mathrm{H}$ & 0.737049 & -9.281999 & 17.079050 \\
\hline 11 & 0.611387 & -10.635072 & 15.929906 \\
\hline 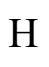 & 0.271150 & -11.935906 & 4.824397 \\
\hline $\mathrm{H}$ & 1.402395 & -10.759790 & 5.525797 \\
\hline I & 4.129278 & -13.855312 & 14.964875 \\
\hline $\mathrm{H}$ & 4.790539 & -15.105292 & 13.914600 \\
\hline 1 & 3.100596 & -15.177926 & 14.404229 \\
\hline $\mathrm{H}$ & 0.094971 & -14.806515 & 12.212807 \\
\hline $\mathrm{H}$ & -0.158802 & -15.616159 & 13.763447 \\
\hline $\mathrm{H}$ & 1.449732 & -15.588304 & 13.031198 \\
\hline $\mathrm{H}$ & 3.097999 & -9.854932 & 16.905408 \\
\hline $\mathrm{H}$ & 3.957222 & -9.340520 & 15.437124 \\
\hline $\mathrm{H}$ & 1.323609 & -16.113697 & 10.510745 \\
\hline $\mathrm{H}$ & 0.728733 & -16.930731 & 9.058103 \\
\hline $\mathrm{H}$ & 0.981533 & -15.174820 & 9.061445 \\
\hline $\mathrm{H}$ & 0.337409 & -8.115695 & 9.645035 \\
\hline H & -0.951144 & -7.159482 & 8.915626 \\
\hline $\mathrm{H}$ & -0.074791 & -8.364703 & 7.952381 \\
\hline $\mathrm{H}$ & -2.172940 & -7.502936 & 14.851058 \\
\hline $\mathrm{H}$ & 5.747427 & -16.087552 & 9.766060 \\
\hline $\mathrm{H}$ & 4.565997 & -12.285629 & 11.494459 \\
\hline $\mathrm{H}$ & 8.143897 & -10.908012 & 12.824264 \\
\hline $\mathrm{H}$ & 5.017849 & -12.078622 & 13.181541 \\
\hline $\mathrm{H}$ & -0.996409 & -13.755975 & 8.826478 \\
\hline $\mathrm{H}$ & -2.519487 & -14.219730 & 9.607676 \\
\hline $\mathrm{H}$ & -1.134840 & -13.689750 & $\begin{array}{c}10.580950 \\
\text { s86 }\end{array}$ \\
\hline
\end{tabular}




\begin{tabular}{|c|c|c|c|}
\hline $\mathrm{H}$ & -2.305308 & -5.636243 & 11.933652 \\
\hline $\mathrm{H}$ & 4.380289 & -17.875703 & 9.290927 \\
\hline $\mathrm{H}$ & 2.730010 & -18.426884 & 9.588498 \\
\hline $\mathrm{H}$ & 3.528427 & -17.372587 & 10.757447 \\
\hline $\mathrm{H}$ & -2.951185 & -9.904642 & 13.113519 \\
\hline $\mathrm{H}$ & -3.201929 & -9.734225 & 14.856990 \\
\hline $\mathrm{H}$ & -1.565870 & -9.922561 & 14.200017 \\
\hline $\mathrm{H}$ & 6.484596 & -13.477478 & 8.347090 \\
\hline 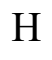 & 7.635482 & -14.641171 & 9.033572 \\
\hline $\mathrm{H}$ & 6.511319 & -13.759477 & 10.081647 \\
\hline $\mathrm{H}$ & 5.079283 & -16.890008 & 7.412512 \\
\hline $\mathrm{H}$ & 6.809333 & -16.662762 & 7.678852 \\
\hline $\mathrm{H}$ & 5.894549 & -15.459998 & 6.774137 \\
\hline $\mathrm{H}$ & -2.561892 & -11.860801 & 11.698733 \\
\hline $\mathrm{H}$ & -3.940341 & -12.467974 & 10.773321 \\
\hline $\mathrm{H}$ & -3.516510 & -10.753212 & 10.708747 \\
\hline $\mathrm{H}$ & -3.843386 & -6.288873 & 13.481155 \\
\hline $\mathrm{H}$ & -4.598186 & -7.766756 & 14.082033 \\
\hline $\mathrm{H}$ & -4.032864 & -7.685301 & 12.411827 \\
\hline $\mathrm{H}$ & -2.488992 & -9.383460 & 7.375624 \\
\hline $\mathrm{H}$ & -3.174487 & -8.134481 & 8.411801 \\
\hline $\mathrm{H}$ & -3.556070 & -9.827142 & 8.712252 \\
\hline $\mathrm{H}$ & -2.104387 & -5.239289 & 14.473302 \\
\hline $\mathrm{H}$ & -1.903690 & -3.810960 & 13.454637 \\
\hline $\mathrm{H}$ & -0.492713 & -4.589081 & 14.165745 \\
\hline $\mathrm{H}$ & -0.049429 & -6.906935 & 15.278170 \\
\hline $\mathrm{H}$ & 1.101463 & -6.021653 & 14.282938 \\
\hline $\mathrm{H}$ & 0.695046 & -5.032563 & 11.915915 \\
\hline $\mathrm{H}$ & -0.579798 & -3.997465 & 11.245590 \\
\hline 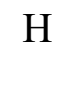 & -0.252885 & -5.592771 & $\begin{array}{c}10.545815 \\
\text { s87 }\end{array}$ \\
\hline
\end{tabular}




\begin{tabular}{|c|c|c|c|}
\hline $\mathrm{H}$ & 4.409071 & -10.217991 & 4.959980 \\
\hline $\mathrm{H}$ & 4.989762 & -9.970549 & 3.302339 \\
\hline $\mathrm{H}$ & 8.405621 & -10.922668 & 6.808828 \\
\hline $\mathrm{H}$ & 9.031276 & -9.692996 & 5.712878 \\
\hline $\mathrm{H}$ & 6.466230 & -2.108204 & 9.650301 \\
\hline$\Pi$ & 4.421197 & -3.912404 & 7.359692 \\
\hline $\mathrm{H}$ & 10.520742 & -8.052425 & 6.903799 \\
\hline $\mathrm{H}$ & 4.365911 & -7.693084 & 3.268617 \\
\hline $\mathrm{H}$ & 3.079363 & -8.455233 & 4.198865 \\
\hline $\mathrm{H}$ & 8.087556 & -3.588124 & 10.910363 \\
\hline $\mathrm{H}$ & 8.913131 & -2.389020 & 9.897525 \\
\hline $\mathrm{H}$ & 8.801313 & -4.078492 & 9.376665 \\
\hline $\mathrm{H}$ & 4.866235 & -6.537606 & 14.469190 \\
\hline $\mathrm{H}$ & 5.793431 & -5.497627 & 15.566859 \\
\hline $\mathrm{H}$ & 3.760685 & -3.359997 & 11.539002 \\
\hline $\mathrm{H}$ & 5.369853 & -2.689822 & 11.798590 \\
\hline $\mathrm{H}$ & 4.871557 & -5.493273 & 3.021191 \\
\hline $\mathrm{H}$ & 6.498714 & -7.448117 & 3.847697 \\
\hline $\mathrm{H}$ & 7.218703 & -8.872165 & 3.043403 \\
\hline $\mathrm{H}$ & 7.985669 & -8.161678 & 4.483786 \\
\hline $\mathrm{H}$ & 9.668034 & -8.801838 & 10.265312 \\
\hline $\mathrm{H}$ & 7.983755 & -4.852340 & 13.649600 \\
\hline $\mathrm{H}$ & 7.051069 & -3.594637 & 14.510924 \\
\hline $\mathrm{H}$ & 7.191017 & -3.564515 & 12.736530 \\
\hline $\mathrm{H}$ & 7.494049 & -10.902443 & 4.345712 \\
\hline $\mathrm{H}$ & 6.370201 & -11.168899 & 5.696693 \\
\hline $\mathrm{H}$ & 3.641457 & -2.090627 & 9.729037 \\
\hline $\mathrm{H}$ & 2.978918 & -2.022421 & 8.098645 \\
\hline $\mathrm{H}$ & 4.667412 & -1.607058 & 8.374193 \\
\hline $\mathrm{H}$ & 7.668407 & -3.451034 & $\begin{array}{c}7.163004 \\
588\end{array}$ \\
\hline
\end{tabular}




\begin{tabular}{|c|c|c|c|}
\hline $\mathrm{H}$ & 7.923615 & -1.765014 & 7.627368 \\
\hline $\mathrm{H}$ & 6.314326 & -2.318422 & 7.151841 \\
\hline $\mathrm{H}$ & 4.696933 & -3.352831 & 13.990390 \\
\hline H & 3.836746 & -4.785212 & 13.384844 \\
\hline $\mathrm{H}$ & 6.415730 & -3.879742 & 5.119396 \\
\hline $\mathrm{H}$ & 6.983229 & -4.338276 & 3.507644 \\
\hline $\mathrm{H}$ & 6.773530 & -5.567008 & 4.769209 \\
\hline $\mathrm{H}$ & 7.491062 & -9.906189 & 10.437710 \\
\hline $\mathrm{H}$ & 8.776002 & -11.105877 & 10.570999 \\
\hline $\mathrm{H}$ & 7.847657 & -10.950715 & 9.067242 \\
\hline $\mathrm{H}$ & 9.932600 & -6.373768 & 14.337283 \\
\hline $\mathrm{H}$ & 1.981301 & -4.475885 & 4.662483 \\
\hline $\mathrm{H}$ & 3.204566 & -5.705656 & 8.522677 \\
\hline $\mathrm{H}$ & 2.138322 & -4.341125 & 8.200765 \\
\hline $\mathrm{H}$ & 2.769837 & -4.617364 & 9.837126 \\
\hline $\mathrm{H}$ & 8.833605 & -6.720071 & 5.631172 \\
\hline $\mathrm{H}$ & 10.298370 & -5.759823 & 5.898663 \\
\hline $\mathrm{H}$ & 8.847764 & -5.480171 & 6.882120 \\
\hline $\mathrm{H}$ & 10.153751 & -9.811752 & 13.743594 \\
\hline $\mathrm{H}$ & 3.324693 & -3.552376 & 3.047104 \\
\hline $\mathrm{H}$ & 4.967932 & -2.943891 & 2.837031 \\
\hline $\mathrm{H}$ & 4.188554 & -2.842088 & 4.417450 \\
\hline $\mathrm{H}$ & 10.777557 & -6.052582 & 11.410091 \\
\hline $\mathrm{H}$ & 10.931407 & -4.847488 & 12.697523 \\
\hline $\mathrm{H}$ & 9.333760 & -5.287176 & 12.063648 \\
\hline $\mathrm{H}$ & 1.272578 & -7.316003 & 5.555812 \\
\hline $\mathrm{H}$ & 0.117895 & -6.010407 & 5.224297 \\
\hline $\mathrm{H}$ & 1.261017 & -5.884196 & 6.572987 \\
\hline $\mathrm{H}$ & 2.643458 & -5.595047 & 2.451530 \\
\hline $\mathrm{H}$ & 0.917476 & -5.624192 & $\begin{array}{c}2.818945 \\
\text { s89 }\end{array}$ \\
\hline
\end{tabular}




\begin{tabular}{|c|c|c|c|}
\hline $\mathrm{H}$ & 1.889457 & -7.075433 & 3.047215 \\
\hline $\mathrm{H}$ & 10.305619 & -5.836984 & 9.020197 \\
\hline $\mathrm{H}$ & 11.695735 & -6.086075 & 7.955212 \\
\hline $\mathrm{H}$ & 11.283536 & -7.298510 & 9.173592 \\
\hline $\mathrm{H}$ & 11.645695 & -8.168928 & 14.355031 \\
\hline $\mathrm{H}$ & 12.377208 & -6.712193 & 13.678992 \\
\hline $\mathrm{H}$ & 11.868112 & -8.017647 & 12.606110 \\
\hline $\mathrm{H}$ & 10.282820 & -10.710978 & 7.917270 \\
\hline $\mathrm{H}$ & 10.987980 & -10.770098 & 9.530082 \\
\hline $\mathrm{H}$ & 11.343038 & -9.409722 & 8.469849 \\
\hline $\mathrm{H}$ & 9.896841 & -8.202241 & 15.752208 \\
\hline $\mathrm{H}$ & 9.751292 & -9.925060 & 16.111562 \\
\hline $\mathrm{H}$ & 8.306601 & -8.923036 & 16.013681 \\
\hline $\mathrm{H}$ & 7.845263 & -6.553761 & 15.067150 \\
\hline $\mathrm{H}$ & 6.689673 & -7.880993 & 15.038271 \\
\hline $\mathrm{H}$ & 7.148176 & -10.253095 & 14.117158 \\
\hline $\mathrm{H}$ & 8.416155 & -11.436137 & 14.493369 \\
\hline $\mathrm{H}$ & 5.632796 & -13.463002 & 12.258049 \\
\hline
\end{tabular}

Complex-Inter-B

$\begin{array}{cccc}\mathrm{U} & 3.275208 & -3.947337 & 20.686879 \\ \mathrm{U} & 4.099311 & -3.713714 & 16.044676 \\ \mathrm{U} & 4.144121 & -7.534227 & 19.992480 \\ \mathrm{U} & 1.722402 & -6.621242 & 16.016615 \\ \mathrm{Rh} & 2.446332 & -4.755091 & 14.037036 \\ \mathrm{Rh} & 3.930642 & -6.099607 & 22.390345 \\ \mathrm{Cl} & 4.653200 & -2.566865 & 18.619416 \\ \mathrm{Cl} & 3.091911 & -8.594439 & 17.539040 \\ \mathrm{P} & 2.184011 & -7.683060 & 22.516329 \\ \mathrm{P} & 7.178917 & -8.342196 & 20.012745 \\ & & & 590\end{array}$




\begin{tabular}{|c|c|c|c|}
\hline $\mathrm{P}$ & -0.048052 & -3.428948 & 21.728467 \\
\hline P & 6.923780 & -4.075440 & 14.761978 \\
\hline $\mathrm{P}$ & 1.521102 & -2.632258 & 14.497002 \\
\hline p & 3.384827 & -6.742733 & 13.232906 \\
\hline $\mathrm{P}$ & 5.781084 & -4.686115 & 22.621169 \\
\hline$r$ & -1.597201 & -5.707899 & 16.346732 \\
\hline$N$ & 3.721617 & -11.248265 & 20.371286 \\
\hline $\mathrm{N}$ & 2.410841 & -8.706494 & 21.175704 \\
\hline $\mathrm{N}$ & 5.795651 & -9.272063 & 19.661786 \\
\hline $\mathrm{N}$ & 5.229009 & -3.268736 & 21.878742 \\
\hline $\mathrm{N}$ & 3.601922 & -1.161305 & 21.661830 \\
\hline$N$ & 1.291856 & -2.598871 & 21.009479 \\
\hline $\mathrm{N}$ & 6.262962 & -2.736346 & 15.578617 \\
\hline $\mathrm{N}$ & 4.994734 & -0.071617 & 15.580453 \\
\hline $\mathrm{N}$ & 2.635463 & -1.877601 & 15.540078 \\
\hline $\mathrm{N}$ & 2.642536 & -7.897344 & 14.225416 \\
\hline $\mathrm{N}$ & 0.545395 & -9.224889 & 15.210973 \\
\hline $\mathrm{N}$ & 3.405724 & -5.638660 & 18.878953 \\
\hline $\mathrm{N}$ & 3.192463 & -5.265391 & 17.508621 \\
\hline $\mathrm{N}$ & -0.576413 & -7.085077 & 16.555748 \\
\hline $\mathrm{C}$ & 8.180844 & -4.617229 & 12.261303 \\
\hline $\mathrm{C}$ & 3.529687 & -5.877037 & 10.570487 \\
\hline $\mathrm{C}$ & -1.256505 & -3.571780 & 24.295184 \\
\hline $\mathrm{C}$ & 0.593262 & -2.415395 & 11.843900 \\
\hline $\mathrm{C}$ & 8.668842 & -4.299978 & 22.748289 \\
\hline $\mathrm{C}$ & -0.233763 & -7.060673 & 21.275620 \\
\hline $\mathrm{C}$ & 6.532211 & -5.726223 & 25.122525 \\
\hline $\mathrm{C}$ & 4.798268 & -3.911613 & 25.165185 \\
\hline $\mathrm{C}$ & 6.063101 & -4.416796 & 24.481149 \\
\hline $\mathrm{C}$ & 2.411726 & -7.742371 & $\begin{array}{c}25.321312 \\
\text { s91 }\end{array}$ \\
\hline
\end{tabular}




\begin{tabular}{|c|c|c|c|}
\hline $\mathrm{C}$ & 3.919027 & -9.297858 & 24.024738 \\
\hline $\mathrm{C}$ & 2.535974 & -8.661253 & 24.103604 \\
\hline t & 7.279717 & -9.449981 & 22.609825 \\
\hline $\mathrm{C}$ & 7.632973 & -8.264287 & 17.255935 \\
\hline $\mathrm{C}$ & 9.277847 & -9.736019 & 18.460547 \\
\hline $\mathrm{C}$ & 8.410805 & -8.476386 & 18.550521 \\
\hline$C$ & 3.945208 & -12.290655 & 21.347727 \\
\hline $\mathrm{C}$ & 1.726328 & -9.991413 & 21.145357 \\
\hline $\mathrm{C}$ & 2.319255 & -10.970894 & 20.131185 \\
\hline $\mathrm{C}$ & 4.563315 & -11.377209 & 19.195363 \\
\hline $\mathrm{C}$ & 5.917746 & -10.690582 & 19.364753 \\
\hline$C$ & -0.515007 & -8.328562 & 23.425612 \\
\hline $\mathrm{C}$ & 0.330750 & -7.297776 & 22.671003 \\
\hline $\mathrm{C}$ & 9.442124 & -8.484830 & 21.740005 \\
\hline $\mathrm{C}$ & 8.158454 & -9.237411 & 21.382563 \\
\hline $\mathrm{C}$ & -1.989651 & -1.162244 & 21.899757 \\
\hline $\mathrm{C}$ & -1.813327 & -2.414735 & 19.740043 \\
\hline $\mathrm{C}$ & -1.675203 & -2.517991 & 21.255969 \\
\hline $\mathrm{C}$ & 1.244390 & -3.604579 & 24.231098 \\
\hline $\mathrm{C}$ & -0.011474 & -3.011826 & 23.600022 \\
\hline $\mathrm{C}$ & 5.010588 & -0.874198 & 21.404545 \\
\hline $\mathrm{C}$ & 5.884455 & -1.980967 & 22.019781 \\
\hline $\mathrm{C}$ & 3.285531 & -0.843238 & 23.050023 \\
\hline $\mathrm{C}$ & 2.650742 & -0.530932 & 20.754009 \\
\hline $\mathrm{C}$ & 1.261982 & -1.138241 & 20.962739 \\
\hline $\mathrm{C}$ & 9.815832 & -3.754025 & 15.283251 \\
\hline $\mathrm{C}$ & 8.323624 & -4.624232 & 17.112615 \\
\hline $\mathrm{C}$ & 8.565752 & -4.578235 & 15.606497 \\
\hline$C$ & 5.192597 & 0.891673 & 14.521098 \\
\hline $\mathrm{C}$ & -0.882924 & -0.986798 & $\begin{array}{c}14.72318 \\
\text { s92 }\end{array}$ \\
\hline
\end{tabular}




\begin{tabular}{|c|c|c|c|}
\hline $\mathrm{C}$ & 6.392516 & -2.835222 & 12.293288 \\
\hline$C$ & 7.540681 & -3.480548 & 13.060429 \\
\hline 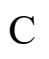 & 7.021098 & -1.501785 & 15.706992 \\
\hline $\mathrm{C}$ & 6.207725 & -0.362866 & 16.323144 \\
\hline $\mathrm{C}$ & 3.800948 & 0.166316 & 16.366411 \\
\hline $\mathrm{C}$ & 2.546095 & -0.437746 & 15.736279 \\
\hline$C$ & -0.192339 & -2.395115 & 16.685078 \\
\hline $\mathrm{C}$ & -0.225929 & -2.298660 & 15.163874 \\
\hline $\mathrm{C}$ & 2.940501 & -1.603571 & 12.291385 \\
\hline$C$ & 1.520384 & -1.711362 & 12.836754 \\
\hline $\mathrm{C}$ & 5.699873 & -7.569507 & 14.541914 \\
\hline$C$ & 5.637853 & -8.217544 & 12.116031 \\
\hline $\mathrm{C}$ & 5.234596 & -7.160009 & 13.148886 \\
\hline $\mathrm{C}$ & -0.364098 & -9.248405 & 14.071255 \\
\hline $\mathrm{C}$ & 1.337170 & -6.885396 & 11.267573 \\
\hline $\mathrm{C}$ & 2.852908 & -6.954899 & 11.422381 \\
\hline $\mathrm{C}$ & 2.641223 & -9.321329 & 13.942254 \\
\hline $\mathrm{C}$ & 1.713118 & -10.053600 & 14.927509 \\
\hline $\mathrm{C}$ & -0.165282 & -9.528505 & 16.446848 \\
\hline $\mathrm{C}$ & -1.164794 & -8.407217 & 16.750122 \\
\hline $\mathrm{C}$ & -2.448784 & -6.025905 & 19.012693 \\
\hline $\mathrm{C}$ & -4.147129 & -6.859874 & 17.374921 \\
\hline $\mathrm{C}$ & -3.029492 & -5.838003 & 17.614863 \\
\hline $\mathrm{C}$ & 7.616505 & -4.724100 & 20.510865 \\
\hline $\mathrm{C}$ & 7.544651 & -5.028390 & 22.004054 \\
\hline $\mathrm{C}$ & -1.637237 & -5.856667 & 13.523272 \\
\hline $\mathrm{C}$ & -2.581789 & -5.942664 & 14.720057 \\
\hline $\mathrm{C}$ & -3.659133 & -4.859096 & 14.595647 \\
\hline 11 & 5.937690 & -0.655446 & 17.340095 \\
\hline $\mathrm{H}$ & 6.861123 & 0.527706 & $\begin{array}{c}16.409821 \\
\text { s93 }\end{array}$ \\
\hline
\end{tabular}




\begin{tabular}{|c|c|c|c|}
\hline $\mathrm{H}$ & 1.702727 & -0.161476 & 16.391528 \\
\hline $\mathrm{H}$ & 2.356477 & 0.075172 & 14.778676 \\
\hline $\mathrm{H}$ & 3.197569 & -7.943326 & 11.094801 \\
\hline $\mathrm{H}$ & 5.714818 & -6.206675 & 12.899897 \\
\hline $\mathrm{H}$ & 1.136407 & -0.701802 & 13.026895 \\
\hline $\mathrm{H}$ & 7.403309 & -1.173283 & 14.725064 \\
\hline $\mathrm{H}$ & 7.910365 & -1.628980 & 16.347084 \\
\hline $\mathrm{H}$ & 0.832325 & -7.666025 & 11.839536 \\
\hline $\mathrm{H}$ & 1.054772 & -7.002243 & 10.213578 \\
\hline $\mathrm{H}$ & 0.947078 & -5.922215 & 11.615183 \\
\hline $\mathrm{H}$ & 0.574884 & -9.599760 & 17.249796 \\
\hline $\mathrm{H}$ & -0.699418 & -10.496399 & 16.399354 \\
\hline $\mathrm{H}$ & 3.639368 & -9.778594 & 14.035698 \\
\hline $\mathrm{H}$ & 2.317307 & -9.517177 & 12.906180 \\
\hline $\mathrm{H}$ & 8.305199 & -2.717390 & 13.253312 \\
\hline $\mathrm{H}$ & 6.013937 & 0.578648 & 13.868811 \\
\hline $\mathrm{H}$ & 5.430781 & 1.905642 & 14.903016 \\
\hline $\mathrm{H}$ & 4.295873 & 0.971616 & 13.898936 \\
\hline $\mathrm{H}$ & -0.823963 & -3.139233 & 14.791744 \\
\hline $\mathrm{H}$ & -1.180870 & -8.542211 & 14.212293 \\
\hline $\mathrm{H}$ & -0.791293 & -10.256658 & 13.925482 \\
\hline $\mathrm{H}$ & 0.171628 & -8.969963 & 13.163640 \\
\hline $\mathrm{H}$ & 3.617286 & 1.246940 & 16.535541 \\
\hline $\mathrm{H}$ & 3.938052 & -0.288031 & 17.350851 \\
\hline $\mathrm{H}$ & 5.117403 & -9.166790 & 12.279743 \\
\hline $\mathrm{H}$ & 6.712555 & -8.421175 & 12.202310 \\
\hline $\mathrm{H}$ & 5.451836 & -7.907395 & 11.084423 \\
\hline $\mathrm{H}$ & 3.194619 & -4.879468 & 10.877173 \\
\hline $\mathrm{H}$ & 3.268241 & -6.004016 & 9.512713 \\
\hline $\mathrm{H}$ & 4.620817 & -5.894134 & $\begin{array}{c}10.645760 \\
\text { s94 }\end{array}$ \\
\hline
\end{tabular}




\begin{tabular}{|c|c|c|c|}
\hline $\mathrm{H}$ & 1.428025 & -11.038548 & 14.515145 \\
\hline $\mathrm{H}$ & 2.242325 & -10.226270 & 15.864807 \\
\hline $\mathrm{H}$ & 5.593850 & -3.560304 & 12.098381 \\
\hline $\mathrm{H}$ & 6.738036 & -2.449976 & 11.325422 \\
\hline $\mathrm{H}$ & 5.951719 & -2.008584 & 12.855973 \\
\hline $\mathrm{H}$ & 0.314601 & -3.296836 & 17.036185 \\
\hline $\mathrm{H}$ & -1.216133 & -2.427928 & 17.067414 \\
\hline $\mathrm{H}$ & 0.312255 & -1.531718 & 17.127559 \\
\hline $\mathrm{H}$ & -3.063088 & -6.930129 & 14.730324 \\
\hline $\mathrm{H}$ & 8.731921 & -5.606061 & 15.253179 \\
\hline $\mathrm{H}$ & 5.439426 & -6.822701 & 15.296466 \\
\hline $\mathrm{H}$ & 7.025656 & -7.355829 & 17.285216 \\
\hline $\mathrm{H}$ & 5.256327 & -8.518424 & 14.853730 \\
\hline $\mathrm{H}$ & 3.626730 & -1.190452 & 13.035311 \\
\hline $\mathrm{H}$ & 2.965020 & -0.967472 & 11.397385 \\
\hline $\mathrm{H}$ & 3.325008 & -2.590183 & 12.009129 \\
\hline $\mathrm{H}$ & -3.470917 & -4.832483 & 17.560416 \\
\hline $\mathrm{H}$ & 9.000605 & -5.104972 & 12.797600 \\
\hline $\mathrm{H}$ & 8.586745 & -4.238891 & 11.314769 \\
\hline $\mathrm{H}$ & 7.446213 & -5.390160 & 12.012644 \\
\hline $\mathrm{H}$ & -1.248387 & -4.839325 & 13.405842 \\
\hline $\mathrm{H}$ & -2.156218 & -6.121783 & 12.592862 \\
\hline $\mathrm{H}$ & -0.774919 & -6.520544 & 13.622311 \\
\hline $\mathrm{H}$ & 8.152039 & -3.622794 & 17.518469 \\
\hline $\mathrm{H}$ & 9.188768 & -5.057492 & 17.628594 \\
\hline $\mathrm{H}$ & 7.447818 & -5.226276 & 17.370783 \\
\hline $\mathrm{H}$ & 10.058987 & -3.745065 & 14.217354 \\
\hline $\mathrm{H}$ & 10.683898 & -4.175100 & 15.807556 \\
\hline $\mathrm{H}$ & 9.713998 & -2.715171 & 15.610424 \\
\hline $\mathrm{H}$ & 0.950211 & -3.431123 & $\begin{array}{c}11.636491 \\
\text { s95 }\end{array}$ \\
\hline
\end{tabular}




\begin{tabular}{|c|c|c|c|}
\hline $\mathrm{H}$ & 0.565167 & -1.872454 & 10.890836 \\
\hline $\mathrm{H}$ & -0.435660 & -2.497895 & 12.207405 \\
\hline $\mathrm{H}$ & -4.432445 & -4.927943 & 15.364734 \\
\hline $\mathrm{H}$ & -4.158901 & -4.923681 & 13.620850 \\
\hline $\mathrm{H}$ & -3.213272 & -3.859177 & 14.665322 \\
\hline $\mathrm{H}$ & -0.296638 & -0.113415 & 15.026178 \\
\hline $\mathrm{H}$ & -1.866189 & -0.895240 & 15.201922 \\
\hline $\mathrm{H}$ & -1.044790 & -0.925666 & 13.643794 \\
\hline $\mathrm{H}$ & -4.605474 & -6.782718 & 16.385775 \\
\hline $\mathrm{H}$ & -4.946170 & -6.711094 & 18.113435 \\
\hline $\mathrm{H}$ & -3.788209 & -7.885625 & 17.495772 \\
\hline $\mathrm{H}$ & -2.059339 & -8.546044 & 16.123675 \\
\hline $\mathrm{H}$ & -1.505868 & -8.554290 & 17.786397 \\
\hline $\mathrm{H}$ & -2.108625 & -7.054331 & 19.155933 \\
\hline $\mathrm{H}$ & -3.205568 & -5.819253 & 19.779922 \\
\hline $\mathrm{H}$ & -1.590174 & -5.378552 & 19.203502 \\
\hline $\mathrm{H}$ & 2.991581 & -0.714408 & 19.730141 \\
\hline $\mathrm{H}$ & 2.597191 & 0.565332 & 20.895747 \\
\hline $\mathrm{H}$ & 6.862061 & -1.940842 & 21.513530 \\
\hline $\mathrm{H}$ & 6.085568 & -1.753203 & 23.080345 \\
\hline $\mathrm{H}$ & 1.780976 & -9.453419 & 24.176514 \\
\hline $\mathrm{H}$ & 0.292102 & -6.346956 & 23.216250 \\
\hline $\mathrm{H}$ & 6.852471 & -3.662539 & 24.586263 \\
\hline $\mathrm{H}$ & 0.827299 & -0.715079 & 21.880722 \\
\hline $\mathrm{H}$ & 0.627922 & -0.771501 & 20.141128 \\
\hline $\mathrm{H}$ & 4.035690 & -9.895934 & 23.117135 \\
\hline $\mathrm{H}$ & 4.102993 & -9.939348 & 24.896022 \\
\hline $\mathrm{H}$ & 4.697846 & -8.526754 & 24.004395 \\
\hline $\mathrm{H}$ & 4.055978 & -10.912469 & 18.346817 \\
\hline $\mathrm{H}$ & 4.735724 & -12.438039 & $\begin{array}{c}18.925264 \\
\text { s96 }\end{array}$ \\
\hline
\end{tabular}




\begin{tabular}{|c|c|c|c|}
\hline $\mathrm{H}$ & 0.658629 & -9.883488 & 20.889422 \\
\hline $\mathrm{H}$ & 1.746232 & -10.467855 & 22.139776 \\
\hline $\mathrm{H}$ & 0.008761 & -1.920329 & 23.724675 \\
\hline $\mathrm{H}$ & 2.289044 & -1.192517 & 23.315059 \\
\hline $\mathrm{H}$ & 3.335693 & 0.245934 & 23.227226 \\
\hline $\mathrm{H}$ & 4.001649 & -1.328672 & 23.713401 \\
\hline $\mathrm{H}$ & 7.663244 & -6.110113 & 22.138043 \\
\hline H & 5.006633 & -12.349537 & 21.608422 \\
\hline $\mathrm{H}$ & 3.628760 & -13.290341 & 20.985705 \\
\hline $\mathrm{H}$ & 3.395103 & -12.080393 & 22.270174 \\
\hline $\mathrm{H}$ & 5.308578 & 0.110432 & 21.808021 \\
\hline 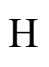 & 5.162550 & -0.849693 & 20.325555 \\
\hline $\mathrm{H}$ & -0.464225 & -9.318339 & 22.961458 \\
\hline $\mathrm{H}$ & -1.567151 & -8.017488 & 23.406559 \\
\hline $\mathrm{H}$ & -0.229977 & -8.434588 & 24.475789 \\
\hline $\mathrm{H}$ & 3.136197 & -6.921488 & 25.260745 \\
\hline $\mathrm{H}$ & 2.615091 & -8.297477 & 26.245613 \\
\hline $\mathrm{H}$ & 1.417627 & -7.294461 & 25.417063 \\
\hline $\mathrm{H}$ & 1.701371 & -11.891744 & 20.138183 \\
\hline $\mathrm{H}$ & 2.220104 & -10.531493 & 19.135858 \\
\hline $\mathrm{H}$ & 1.199427 & -4.698746 & 24.250565 \\
\hline $\mathrm{H}$ & 1.369519 & -3.259225 & 25.265635 \\
\hline $\mathrm{H}$ & 2.150451 & -3.332394 & 23.681475 \\
\hline$\Pi$ & 6.819185 & -5.216358 & 19.949934 \\
\hline $\mathrm{H}$ & 8.570001 & -5.081034 & 20.105391 \\
\hline П & 7.544337 & -3.651101 & 20.315547 \\
\hline $\mathrm{H}$ & 8.432683 & -10.220269 & 20.978387 \\
\hline $\mathrm{H}$ & -2.425537 & -3.239393 & 21.611162 \\
\hline 1 & 0.373648 & -6.364448 & 20.692718 \\
\hline $\mathrm{H}$ & -1.234196 & -6.626330 & $\begin{array}{c}21.356777 \\
\text { s97 }\end{array}$ \\
\hline
\end{tabular}




\begin{tabular}{|c|c|c|c|}
\hline $\mathrm{H}$ & -0.307851 & -7.996755 & 20.714755 \\
\hline $\mathrm{H}$ & 4.470654 & -2.952664 & 24.760367 \\
\hline $\mathrm{H}$ & 4.967195 & -3.785978 & 26.242191 \\
\hline $\mathrm{H}$ & 3.972383 & -4.619323 & 25.031702 \\
\hline $\mathrm{H}$ & 9.075094 & -7.612727 & 18.696857 \\
\hline $\mathrm{H}$ & -2.182480 & -3.089114 & 23.971104 \\
\hline $\mathrm{H}$ & -1.183708 & -3.443107 & 25.382667 \\
\hline $\mathrm{H}$ & -1.359142 & -4.646737 & 24.101306 \\
\hline $\mathrm{H}$ & 6.987856 & -8.492696 & 23.056951 \\
\hline $\mathrm{H}$ & 7.816716 & -10.023635 & 23.376232 \\
\hline $\mathrm{H}$ & 6.362507 & -9.988339 & 22.357929 \\
\hline $\mathrm{H}$ & -1.141616 & -1.653435 & 19.335308 \\
\hline $\mathrm{H}$ & -2.836639 & -2.128619 & 19.464599 \\
\hline $\mathrm{H}$ & -1.579347 & -3.353777 & 19.236692 \\
\hline $\mathrm{H}$ & -1.894949 & -1.163297 & 22.988823 \\
\hline $\mathrm{H}$ & -3.023839 & -0.875855 & 21.666092 \\
\hline $\mathrm{H}$ & -1.344202 & -0.370349 & 21.509930 \\
\hline $\mathrm{H}$ & 5.750083 & -6.491167 & 25.056575 \\
\hline $\mathrm{H}$ & 6.753314 & -5.572341 & 26.185988 \\
\hline $\mathrm{H}$ & 7.431856 & -6.135560 & 24.654171 \\
\hline $\mathrm{H}$ & 10.094450 & -8.321399 & 20.876920 \\
\hline $\mathrm{H}$ & 10.019231 & -9.045525 & 22.485997 \\
\hline $\mathrm{H}$ & 9.220166 & -7.503608 & 22.172652 \\
\hline $\mathrm{H}$ & 8.554739 & -3.212281 & 22.693274 \\
\hline $\mathrm{H}$ & 9.632347 & -4.544765 & 22.283860 \\
\hline $\mathrm{H}$ & 8.740717 & -4.576579 & 23.803351 \\
\hline $\mathrm{H}$ & 9.909881 & -9.884394 & 19.339873 \\
\hline $\mathrm{H}$ & 9.946410 & -9.664765 & 17.592332 \\
\hline $\mathrm{H}$ & 8.673155 & -10.637890 & 18.329334 \\
\hline $\mathrm{H}$ & 6.483982 & -11.210413 & $\begin{array}{c}20.15699 \\
\text { s98 }\end{array}$ \\
\hline
\end{tabular}




$\begin{array}{llll}\mathrm{H} & 6.477610 & -10.867450 & 18.431453 \\ \mathrm{H} & 6.951336 & -9.097379 & 17.060395 \\ \mathrm{H} & 8.319499 & -8.179344 & 16.405064 \\ \mathrm{H} & 6.789558 & -7.679215 & 14.551696\end{array}$

252

\section{Complex 5}

U $\quad 16.092688-3.7653906 .159698$

U $\quad 17.758416-3.4990253 .057888$

U $\quad 13.439711-3.7648903 .835190$

U $\quad 11.773952-3.4999266 .937129$

Rh $18.656437-4.8653785 .837844$

Rh $10.876206-4.8655304 .156682$

P $\quad 20.628262-2.7628930 .883719$

P $\quad 17.863303-5.8232647 .957041$

P $\quad 18.917239-6.4113534 .096199$

P $\quad 19.098903-2.5804246 .110651$

P $\quad 8.904010-2.7648489 .111461$

P $\quad 11.669520-5.8225172 .037230$

P $\quad 10.615491-6.4120855 .897843$

P $\quad 10.433308-2.5805483 .884618$

N $\quad 17.722978-1.9619715 .224501$

$\mathrm{N} \quad 19.101791-2.1101241 .414212$ 

N $\quad 16.310260-5.0842308 .185484$
N $\quad 16.922275-0.7678042 .638558$
N $\quad 16.591311-4.6836370 .745519$
N $\quad 18.415545-5.7051332 .627093$
N $\quad 15.695306-3.7768573 .893194$
N $\quad 11.809079-1.9621244 .771033$
$\mathrm{N} \quad 10.430430-2.1117328 .581261$
N $\quad 13.222397-5.0830881 .808980$
$\mathrm{N} \quad 12.609731-0.7686787 .357379$
N $\quad 12.941374-4.6853299 .248908$
N $11.117081-5.7062797 .367190$
N $\quad 13.837105-3.7770416 .101736$
C $\quad 17.656664-0.0230893 .674638$
C $\quad 11.875135-0.0237776 .321580$
C $\quad 21.579991-5.7914633 .313159$
C $\quad 20.724185-6.9441923 .821174$
C $\quad 18.651001-9.2711893 .354612$
C $\quad 21.320953-7.5824225 .075144$
C $\quad 16.579274-7.9736533 .951535$
C $\quad 18.690633-5.92893310 .806929$
C $\quad 23.208833-2.3775492 .027498$
C $\quad 18.848239-8.5031988 .170882$ 

C $\quad 18.076822-8.1298974 .201430$
C $22.354952-0.3113960 .872894$
C $\quad 16.404806-8.2402727 .590251$
C $21.961336-1.5775331 .636572$
C $\quad 15.488825-5.4225929 .340403$
C $\quad 21.035644-0.4567775 .430714$
C $13.997524-5.7302079 .064838$
C $20.721754-1.9495655 .327726$
C $\quad 18.834597-0.7326461 .043314$
C $\quad 17.582068-7.6704198 .371274$
C $\quad 17.380835-0.3634841 .299038$
C $\quad 17.668360-1.6155738 .368956$
C $20.459623-5.4360159 .078054$
C $\quad 17.404753-0.5421895 .084454$
C $\quad 18.362085-6.3572431 .340348$
C $20.166131-1.7643848 .692812$
C $\quad 17.833708-5.3636960 .307688$
C $\quad 18.976284-5.3097599 .437652$
C $\quad 15.488511-0.5216522 .740478$
C $21.903853-2.7955025 .809041$
C $\quad 19.036656-1.4883527 .700491$
C $\quad 7.952694-5.7924666 .680870$ 

C $8.808552-6.9450546 .172601$
C $\quad 10.881690-9.2722146 .638349$
C $8.211863-7.5829844 .918442$
C $\quad 12.953476-7.9743546 .042334$
C $\quad 6.323449-2.3791777 .967767$
C $\quad 10.685082-8.5025221 .822548$
C $\quad 11.455985-8.1305705 .792078$
C $\quad 7.415115-2.98242111 .481492$
C $7.177111-0.3135219 .123428$
C $\quad 13.128392-8.2393412 .403536$
C $7.570863-1.5792478 .359136$
C $\quad 14.043900-5.4209810 .653968$
C $8.496258-0.4574264 .565231$
C $\quad 8.701814-2.32666110 .970275$
C $\quad 15.535212-5.7285810 .929495$
C $\quad 9.890052-2.77764411 .813583$
C $8.810304-1.9502194 .667628$
C $\quad 10.697441-0.7343538 .952658$
C $\quad 11.951139-7.6694941 .622498$
C $12.151141-0.3648838 .697046$
C $\quad 11.863662-1.6150001 .626474$
C $\quad 9.073103-5.4354890 .916399$ 

C $\quad 13.298398-3.67780210 .247042$
C $\quad 12.127079-0.5423384 .911570$
C $\quad 11.170589-6.3587738 .653739$
C $\quad 9.365847-1.7637091 .302788$
C $\quad 11.699001-5.3654919 .686634$
C $\quad 10.556408-5.3087760 .556817$
C $\quad 14.043434-0.5221557 .255486$
C $\quad 7.628345-2.7960874 .185851$
C $\quad 10.495420-1.4879962 .295098$
C $\quad 22.117205-2.979269-1.486383$
C $20.830424-2.323881-0.974902$
C $\quad 19.642245-2.774624-1.818420$
C $\quad 16.234250-3.675997-0.252488$
C $\quad 10.842194-5.927408-0.812675$
H $\quad 18.722189-0.1329613 .458398$
H $\quad 17.4117401 .0547433 .614905$
H $\quad 21.911387-0.2145694 .815417$
H 20.2134590 .1769595 .087283
H $\quad 17.2307680 .7219091 .149127$
H $\quad 15.2449660 .5536882 .680036$
H $\quad 10.809641-0.1339546 .537828$
H 12.1198291 .0540876 .381670 
H $\quad 7.620439-0.2155725 .180559$

H 9.3183470 .1762554 .908990

H $\quad 12.3010400 .7204858 .847313$

H $\quad 14.2867090 .5532337 .316160$

H $\quad 21.742091-5.0478284 .098191$

H $\quad 22.564427-6.1617292 .997533$

H $\quad 21.117622-5.2783752 .464617$

H $\quad 20.676418-7.6996633 .026463$

H $\quad 19.677037-9.5270153 .632558$

H $\quad 18.044940-10.1753773 .499247$

H $\quad 18.641601-9.0464672 .283802$

H $\quad 20.774007-8.4755605 .394049$

H $22.362031-7.8815914 .897852$

H $\quad 21.313139-6.8746765 .911381$

H $\quad 16.377441-7.8467742 .885172$

H $\quad 16.029671-8.8625394 .283947$

H $\quad 16.166975-7.1010344 .468584$

Н $\quad 18.930540-6.99750310 .832629$

Н $19.312084-5.44775511 .574063$

H $\quad 17.647689-5.81042811 .112654$

H $\quad 23.751464-2.7364151 .145476$

H $\quad 23.908396-1.7571892 .603811$ 
H $\quad 22.954716-3.2546292 .628676$

H $\quad 19.649194-8.2143878 .856164$

Н $\quad 18.635546-9.5652608 .348271$

Н $\quad 19.237470-8.4066767 .153138$

H $\quad 18.222410-8.3964485 .254565$

H 23.0256640 .3057641 .486084

H $\quad 22.897046-0.541515-0.050603$

H $\quad 21.4946520 .3081930 .605148$

H $\quad 16.555246-8.1402586 .514317$

H $\quad 16.275218-9.3068077 .815038$

H $\quad 15.471801-7.7291927 .832286$

H $\quad 21.458940-1.2672992 .562368$

H $\quad 15.860366-6.3397929 .816474$

H $\quad 15.572611-4.65906610 .129271$

H $\quad 21.279184-0.1619386 .456495$

H $\quad 13.733946-6.5544469 .746716$

H $\quad 13.903847-6.1012788 .042244$

H $20.541918-2.2018964 .272326$

H $\quad 19.486482-0.0188431 .578578$

H $\quad 19.016461-0.529005-0.025944$

H $\quad 17.329501-7.7084619 .438609$

H $\quad 16.745806-0.8814770 .571916$ 
H $\quad 17.428200-2.6591028 .621683$

H $\quad 17.630112-1.0467719 .306703$

Н $\quad 16.876510-1.2049437 .729373$

H $\quad 20.662285-5.0125828 .087913$

H $\quad 21.086142-4.9135689 .813427$

H $\quad 20.788621-6.4797579 .064858$

Н $\quad 15.374580-3.0912660 .086514$

H $\quad 17.9785310 .0972535 .769969$

H $\quad 16.350438-0.3600965 .347603$

H $\quad 17.725327-7.2552951 .344728$

H $\quad 19.353103-6.6876730 .983665$

H $\quad 21.142219-1.4798928 .293197$

H $\quad 20.014224-1.1854129 .613008$

H $\quad 20.218952-2.8191148 .970651$

H $\quad 18.592528-4.5896180 .152747$

H $\quad 18.734453-4.2411409 .496063$

H $\quad 15.106152-0.9191123 .683318$

H $\quad 14.976707-1.0326631 .920342$

H $\quad 21.651728-3.8598985 .808669$

H $\quad 22.772118-2.6492135 .156434$

H $\quad 22.210240-2.5260046 .824293$

H $\quad 19.144505-0.4498837 .359933$ 
Н $7.790114-5.0489415 .895826$

H $\quad 6.968474-6.1629206 .996950$

Н $\quad 8.415275-5.2792047 .529192$

H $\quad 8.856276-7.7007436 .967105$

H $\quad 9.855772-9.5280776 .359998$

H $\quad 11.487908-10.1762796 .493600$

H $\quad 10.890728-9.0478567 .709237$

H $8.758865-8.4760204 .599342$

H $\quad 7.170794-7.8822525 .095619$

H $\quad 8.219662-6.8750374 .082377$

H $\quad 13.155082-7.8480177 .108804$

H $\quad 13.503207-8.8630275 .709568$

H $\quad 13.365815-7.1014355 .525821$

H $\quad 5.780816-2.7385248 .849592$

H $\quad 5.623856-1.7585917 .391735$

H $\quad 6.577663-3.2559347 .366158$

H $\quad 9.884223-8.2137951 .137120$

H $\quad 10.897997-9.5645331 .645125$

H $\quad 10.295624-8.4061232 .840218$

H $\quad 11.310607-8.3967414 .738815$

H $\quad 7.457909-4.07151311 .364242$

H $\quad 7.268035-2.76787812 .547885$ 
H $\quad 6.523735-2.63159710 .953576$

H $\quad 6.5063490 .3038758 .510531$

H $\quad 6.635027-0.54414410 .046805$

H $\quad 8.0373460 .3060229 .391495$

H $\quad 12.977749-8.1396723 .479474$

H $\quad 13.258235-9.3057822 .178450$

H $\quad 14.061330-7.7279942 .161814$

H $\quad 8.073278-1.2685167 .433516$

H $\quad 13.672421-6.3380360 .177575$

H $\quad 13.960085-4.657184-0.134630$

H $\quad 8.252769-0.1621893 .539551$

H $\quad 8.604007-1.23897011 .072965$

H $\quad 15.798834-6.5527470 .247551$

H $\quad 15.628902-6.0997131 .952063$

H $\quad 10.822741-2.30807411 .490255$

H $\quad 9.733362-2.51454312 .867794$

H $\quad 10.026511-3.86353511 .767517$

Н $\quad 8.990078-2.2029765 .722937$

H $\quad 10.045457-0.0204538 .417649$

H $\quad 10.515541-0.53112910 .021986$

H $\quad 12.203852-7.7072190 .555182$

H $\quad 12.786270-0.8830119 .423986$ 
H $\quad 12.103815-2.6584411 .373355$

H $11.901844-1.0458750 .688920$

H $\quad 12.655560-1.2045672 .266128$

H $\quad 8.870363-5.0123221 .906638$

H $\quad 8.446438-4.9130350 .181155$

Н $\quad 8.744369-6.4793150 .929374$

Н $\quad 12.447563-3.00451410 .366625$

Н $\quad 13.541896-4.11443311 .229994$

H $\quad 14.158039-3.0929899 .908111$

H $\quad 11.5531750 .0972574 .226305$

H $\quad 13.181357-0.3599854 .648443$

H $\quad 11.807374-7.2568058 .649089$

H $\quad 10.179589-6.6893139 .010370$

H $\quad 8.389815-1.4792371 .702555$

H $\quad 9.517716-1.1845130 .382725$

H $\quad 9.312920-2.8183621 .024675$

H $\quad 11.864921-5.87560010 .652790$

H $\quad 10.940182-4.5914559 .841788$

H $\quad 10.797991-4.2400780 .498781$

H $\quad 14.425880-0.9193406 .312570$

H $\quad 14.555380-1.0332178 .075499$

H $\quad 7.880573-3.8604574 .185826$ 
H $\quad 6.759998-2.6501334 .838426$

H $\quad 7.322038-2.5262243 .170673$

H $\quad 10.387582-0.4496362 .635993$

H $\quad 22.074531-4.068420-1.369637$

H $\quad 22.264289-2.764215-2.552672$

H $23.008531-2.628586-0.958280$

H $\quad 20.928114-1.236137-1.077108$

H $\quad 18.709520-2.305218-1.494956$

H $\quad 19.798955-2.511131-2.872531$

H $\quad 19.505842-3.860538-1.772754$

H $\quad 17.085058-3.002659-0.371977$

H $\quad 15.990775-4.112505-1.235500$

H $\quad 17.667820-5.873571-0.658594$

H $\quad 10.602544-6.996028-0.838741$

H $\quad 10.220617-5.446120-1.579638$

H $\quad 11.885105-5.808545-1.118369$ 


\section{References}

(1) Sheldrick, G. M. Crystal structure refinement with SHELXL. Acta Crystallogr. Sect. C 2015, 71, 3-8.

(2) Dolomanov, O. V.; Bourhis, L. J.; Gildea, R. J.; Howard, J. A. K.; Puschmann, H. OLEX2: A complete structure solution, refinement and analysis program. J. Appl. Crystallogr. 2009, 42, 339-341

(3) Gaussian09, Revision A.02. Frisch, M. J.; Trucks, G.W.; Schlegel, H. B.; Scuseria, G. E.; Robb, M. A.; Cheeseman, J. R.; Scalmani, G.; Barone, V.; Mennucci, B.; Petersson, G. A.; Nakatsuji, H.; Caricato, M.; Li, X.; Hratchian, H. P.; Izmaylov, A. F.; Bloino, J.; Zheng, G.; Sonnenberg, J. L.; Hada, M.; Ehara, M.; Toyota, K.; Fukuda, R.; Hasegawa, J.; Ishida, M.; Nakajima, T.; Honda, Y.; Kitao, O.; Nakai, H.; Vreven, T.; Montgomery, J. A.; Jr., Peralta, J. E.; Ogliaro, F.; Bearpark, M.; Heyd, J. J.; Brothers, E.; Kudin, K. N.; Staroverov, V. N.; Kobayashi, R.; Normand, J.; Raghavachari, K.; Rendell, A.; Burant, J. C.; Iyengar, S. S.; Tomasi, J.; Cossi, M.; Rega, N.; Millam, J. M.; Klene, M.; Knox, J. E.; Cross, J. B.; Bakken, V.; Adamo, C.; Jaramillo, J.; Gomperts, R.; Stratmann, R. E.; Yazyev, O.; Austin, A. J.; Cammi, R.; Pomelli, C.; Ochterski, J. W.; Martin, R. L.; Morokuma, K.; Zakrzewski, V. G.; Voth, G. A.; Salvador, P.; Dannenberg, J. J.; Dapprich, S.; Daniels, A. D.; Farkas, O.; Foresman, J. B.; Ortiz, J. V.; Cioslowski, J.; Fox, D. J. Gaussian, Inc., Wallingford CT, 2009.

(4) Becke, A.D. Density-functional thermochemistry. III. The role of exact Exchange. J. Chem. Phys. 1993, 98, 5648-5652.

(5) Perdew, J. P.; Wang, Y. Accurate and simple analytic representation of the electrongas correlation energy. Phys. Rev. B 1992, 45, 13244-13249.

(6) Cao, X.; Dolg, M. Segmented contraction scheme for small-core actinide pseudopotential basis set. J. Mol. Struct: Theochem 2004, 673, 203-209.

(7) Cao, X.; Dolg, M.; Stoll, H. Valence basis sets for relativistic energyconsistent small-core actinide pseudopotentials. J. Chem. Phys. 2003, 118, 487-496.

(8) Kuechle, W.; Dolg, M.; Stoll, H; Preuss, H. Energy-adjusted pseudopotentials for the actinides. Parameter sets and test calculations for thorium and thorium monoxide. J. Chem. Phys. 1994, 100, 7535-7542. 
(9) Leininger T.; Nicklass, A.; Küchle, W.; Stoll, H.; Dolg, M.; Bergner, A. The accuracy of the pseudopotential approximation: Non-frozen-core effects for spectroscopic constants of alkali fluorides XF (X=K, Rb, Cs). Chem. Phys. Lett. 1996, $255,274-280$.

(10) Dolg, M.; Wedig, U.; Stoll, H; Preuss, H. Energy-adjusted ab initio pseudopotentials for the first row transition elements. J. Chem. Phys. 1987, 86, 866872.

(11) Hehre, W. J.; Ditchfield, R.; Pople, J. A. Self-consistent molecular orbital methods. XII. Further extensions of Gaussian-type basis sets for use in molecular orbital studies of organic molecules. J. Chem. Phys. 1972, 56, 2257-2261.

(12) Hariharan, P. C. \& Pople, J. A. The influence of polarization functions on molecular orbital hydrogenation energies. Theor. Chim. Acta 1973, 28, 213-222.

(13) Schlegel, H. B. Optimization of equilibrium geometries and transition structures. J. Comput. Chem. 1982, 3, 214-218.

(14) Reed, A. E.; Curtiss, L. A.; Weinhold, F. Intermolecular interactions from a natural bond orbital, donor-acceptor viewpoint. Chem. Rev. 1988, 88, 899-926.

(15) Zhurko, G. A. Home Page: a set of graphical tools for facilitating working with quantum chemistry computations (http://www.chemcraftprog.com). 